\title{
DEVELOPMENT OF A HIGH-SPEED UV PARTICLE IMAGE VELOCIMETRY TECHNIQUE AND APPLICATION FOR MEASUREMENTS IN INTERNAL COMBUSTION ENGINES
}

\author{
by \\ Claudia M. Fajardo \\ A dissertation submitted in partial fulfillment \\ of the requirements for the degree of \\ Doctor of Philosophy \\ (Mechanical Engineering) \\ in The University of Michigan \\ 2007
}

Doctoral Committee:

Professor Volker Sick, Chair

Professor Steven L. Ceccio

Professor Werner J. A. Dahm

David L. Reuss, General Motors Corporation 
(C) $\frac{\text { Claudia M. Fajardo }}{\text { All Rights Reserved }} 2007$ 
To my Family 


\section{ACKNOWLEDGMENTS}

I would like to thank my advisor, Professor Volker Sick for introducing me to the fascinating world of optical diagnostics, for his valuable guidance and patience through years of learning and for facilitating opportunities to integrate technical and personal growth through collaborative experiences abroad. I would like to thank members of my doctoral committee: Dr. David Reuss, for his valuable involvement and support, for facilitating insightful discussions and providing advice on the subjects of particle image velocimetry, turbulence and internal combustion engines; Professor Werner J. A. Dahm, for introducing me to the complex and captivating subject of turbulence through two of the most interesting and challenging courses I have taken as an engineering student; and Professor Steven Ceccio, for assisting my first attempts to pursuing UV particle image velocimetry by sharing indispensable equipment.

I would also like to acknowledge the support of my colleagues in the quantitative laser diagnostic laboratory at the University of Michigan, some of whom have already graduated: Drs. Rui Zhang, James Smith and Charles Funk and those who continue to build upon the valuable knowledge our group has accumulated throughout the years: Brian Peterson, Ali Alharbi and Michael Cundy. I am grateful for their support with laboratory equipment and have greatly benefited from their viewpoint during technical discussions. I would like to recognize the help of my colleagues in the Thermodynamic Institute at the University of Karlsruhe, especially Alexander Schubert, Rainer Stauch, Karin Koenig, Dr. Robert Schiessl and Professor Ulrich Maas, for offering technical support and a welcoming environment during my visits to the University of Karlsruhe. 
I am exceptionally grateful to my family, especially to my parents and sister for providing encouragement throughout my years as a student and to my husband for being a source of support and encouragement, for his interest in my work and for promoting technical discussions that widen my knowledge and perspective every day. 
TABLE OF CONTENTS

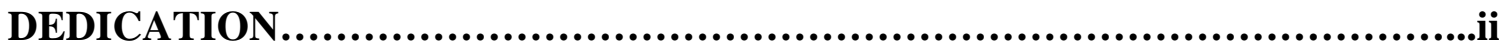

ACKNOWLEDGMENTS .................................................................................ii

LIST OF FIGURES …........................................................................................viii

LIST OF TABLES …................................................................................................ xii

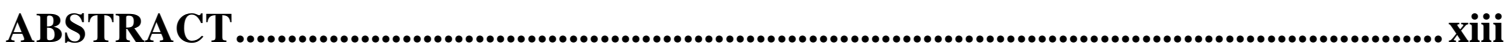

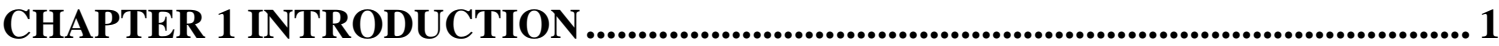

1.1 In-Cylinder Flow in Internal Combustion Engines ..................................... 1

1.2 Experimental Flow Techniques Applied to Internal Combustion Engines.. 6

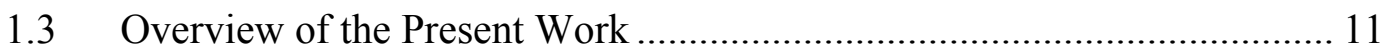

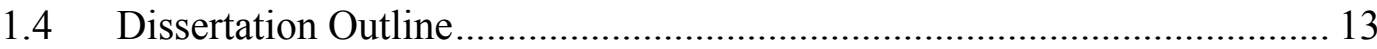

CHAPTER 2 SYSTEMATIC AND RANDOM ERRORS OF PIV

MEASUREMENTS IN INTERNAL COMBUSTION ENGINES ............................. 15

$2.1 \quad$ Particle Image Velocimetry .................................................................. 15

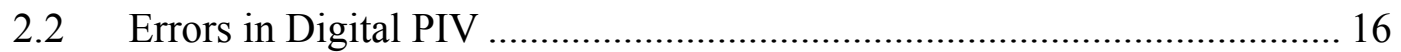

2.3 Parameter Optimization for PIV Experiments in IC Engines .................... 19

CHAPTER 3 PRELIMINARY PIV EXPERIMENTS IN A FIRED SIDI ENGINE22

3.1 Experimental Equipment and Setup .......................................................... 22

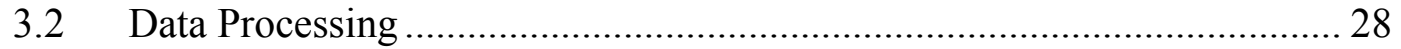

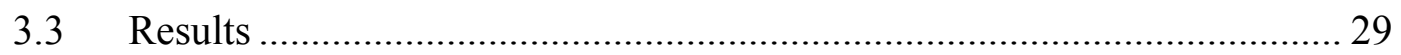

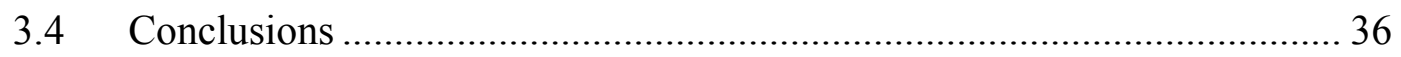

CHAPTER 4 HIGH-SPEED UV PIV USING A SINGLE LASER-CAMERA

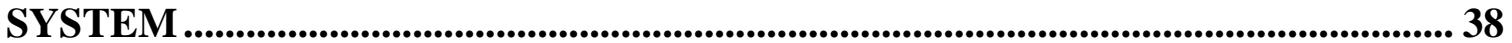

$4.1 \quad$ Experimental Equipment and Setup ………………................................ 38

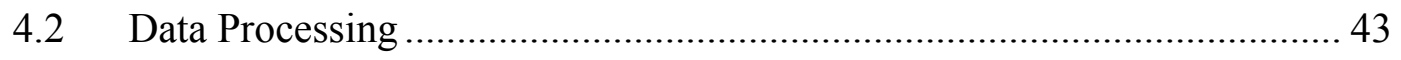

$4.3 \quad$ Results and Discussion....................................................................... 44

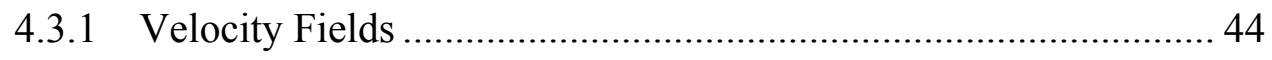




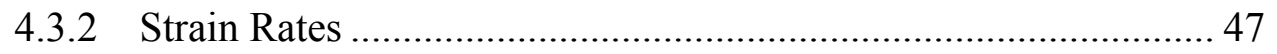

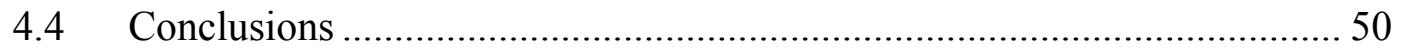

CHAPTER 5 SIMULTANEOUS HIGH-SPEED UV PIV AND PLIF IN A COLD AIR JET TO STUDY TRACER INTERACTION................................................... 52

5.1 Experimental Equipment and Setup ......................................................... 53

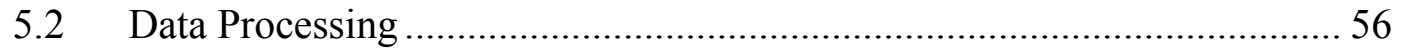

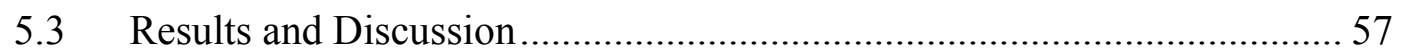

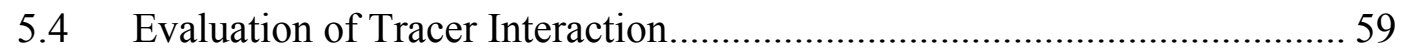

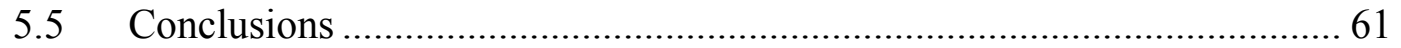

CHAPTER 6 EFFECT OF PIV MEASUREMENT NOISE ON KINETIC ENERGY AND DISSIPATION SPECTRA OF ENGINE FLOWS ...........................................63

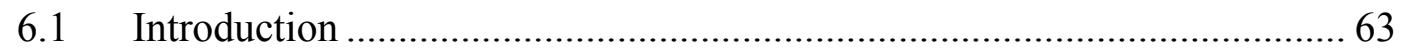

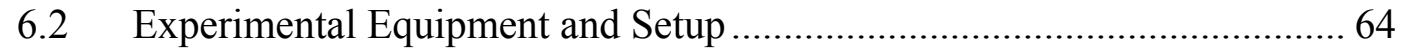

6.3 Image Acquisition and Data Processing ..................................................... 67

6.3.1 Kinetic Energy and Dissipation Rate Spectra Calculations .......... 67

6.4 Kinetic Energy and Dissipation Rate Spectra from Engine Experiments .. 70

6.5 Fixed-displacement PIV experiments ...................................................... 74

6.5.1 Spectra from fixed-displacement experiments ................................ 75

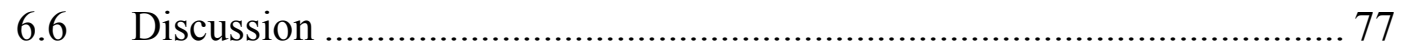

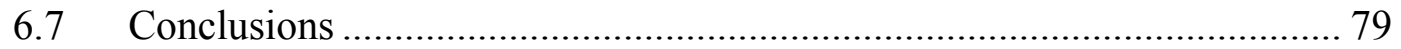

\section{CHAPTER 7 HIGH-SPEED UV PIV USING A TWO LASER-ONE CAMERA}

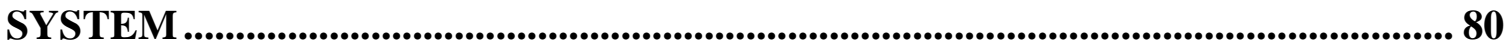

7.1 Experimental Equipment and Setup …………………........................ 80

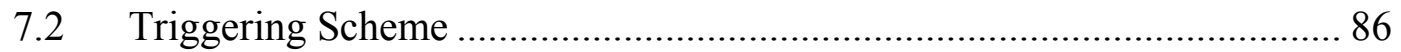

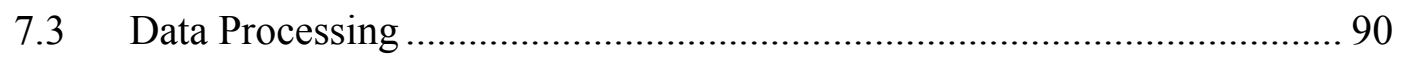

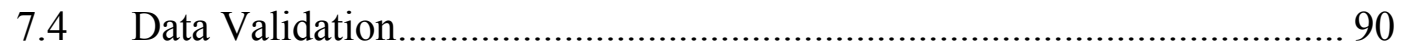

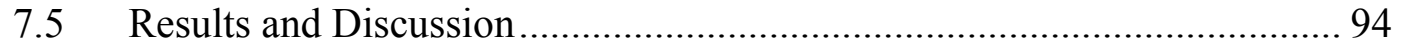

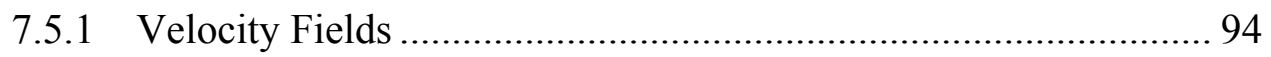

7.5.2 Kinetic Energy …………………………………................... 102

CHAPTER 8 CONCLUSIONS AND FUTURE WORK ........................................... 106

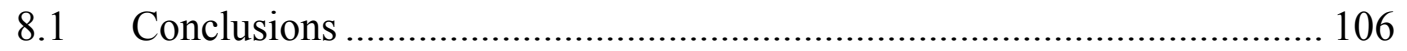


8.2 Future Work ................................................................................. 108

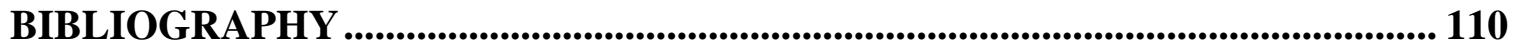




\section{LIST OF FIGURES}

Figure 3-1 Optical engine (a) and piston (b) used in the PIV measurements. The quartz cylinder enables optical access to the engine. The light sheet enters the combustion chamber through the top piston window.................................... 23

Figure 3-2 Experimental setup for preliminary PIV measurements in the optical engine using $532 \mathrm{~nm}$ wavelength excitation. Legend:-M1: HR 355 HT 532 mirrors, BD: beam dump, A: aperture, DL: diverging lens, FO: focusing optics, G: gate, M2: $45^{\circ}$ turning mirror, F: $532 \mathrm{~nm}$ narrow-band filter........................ 25

Figure 3-3 Rate of change of the cylinder volume as the piston approaches TDC .......... 27

Figure 3-4 Instantaneous velocity fields at 600 RPM (a) and 2000 RPM (b). Both images were recorded at $90{ }^{\circ} \mathrm{BTDC}$. Every other vector is shown 31

Figure 3-5 Ensemble average flow fields at 600 RPM (a) and 2000 RPM (b) normalized by the mean piston speed. Every other vector is shown.

Figure 3-6 Instantaneous motored (a-d) and fired (e-g) images of the flow inside the cylinder of a SIDI engine. Substantial soot luminosity prevented the acquisition of images near the spark plug at $25^{\circ} \mathrm{BTDC}$, as shown by the red circle in (h)

Figure 4-1 Transmissivity of the Phantom V7.1 entrance-windows. The built-in windows, used in the current experiment allow $87 \%$ transmissivity at 355 $\mathrm{nm}$.

Figure 4-2 Large field-of-view flow characteristics at $34{ }^{\circ} \mathrm{BTDC}$ (compression), from experimental results presented in Chapter 3. The dotted line indicates the location of the $11 \times 11 \mathrm{~mm}^{2}$ imaging area used in the present experiment with respect to the spark plug and injector.

Figure 4-3 Sequence of instantaneous velocity fields from fuel injection to early flame propagation. Mie scattering signal from the spray prevents velocity field evaluation from $40{ }^{\circ} \mathrm{BTDC}$ to $35^{\circ} \mathrm{BTDC}$. The deformation of the plasma 
channel has been recorded. The dotted line indicates the location of the side piston window. Defocusing prevents imaging in this area. Notice the velocity scale change in (i) and (j)

Figure 4-4 Spatially averaged velocity magnitude in a $5 \mathrm{~mm}^{2}$ area to the left of the spark plug. Legend: * four instantaneous values per crank angle for one of 100 cycles, $\square$ average over all cycles.

Figure 4-5 Spatially averaged sum of the normal strain rates. Legend: * four instantaneous values per crank angle for one of 100 cycles, $\square$ averaged over all cycles. 48

Figure 4-6 Sequence of instantaneous shear strain rate fields for the velocity fields shown in Figure 4-3. No values were computed in the black areas shown. 50

Figure 5-1 Setup for the velocity-scalar measurements in a cold jet using $355 \mathrm{~nm}$ excitation light. Legend: M1-HR355 HT 532 mirror, M2-HR 355 mirror, Tfocusing optics, DL-diverging lens, L1-105 mm UV lens, L2-105 mm Nikon Micro-Nikkor lens, F1-355 nm interference filter, F2-GG 420 filter, I-image intensifier. 54

Figure 5-2 Triggering scheme for the simultaneous application of high-speed PLIF and PIV to a jet flow... 56

Figure 5-3 Velocity-scalar fields obtained with high-speed PIV and biacetyl PLIF ........ 58

Figure 5-4 Average quality (Q) factor and percentage of vectors retained after postprocessing, corresponding to the velocity fields obtained with the singlelaser, single-camera, high-speed UV PIV technique. The Q factor, which represents the ratio of the signal-to-noise peaks in the correlation map, demonstrates the high quality of the PIV measurements. A decrease in Q is noted for areas with rapid changes in spatial extent of the jet. This is due to the adaptive masking scheme that was employed, leading to small areas where no overlapping seeded areas are found. 60

Figure 6-1 Experimental setup for the engine PIV measurements. F: narrow-band filter, O: lightsheet optics, M1,M2: dichroic mirrors, M3: turning mirror, A: aperture 65 
Figure 6-2 Location of the field of view used in the high resolution $(350 \mu \mathrm{m})$ PIV measurements (b) with respect to the field of view corresponding to the low resolution (2 $\mathrm{mm}$ ) experiments (a). Every second vector shown in (a) and

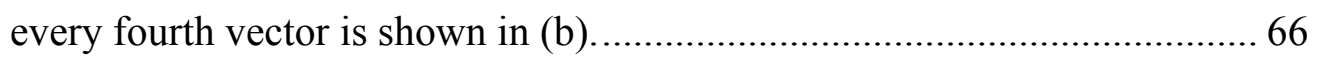

Figure 6-3 Kinetic energy spectra for the high (a) and low (b) swirl flow conditions. The kinetic energy increases near the resolution limit when the $3 \times 3$ smoothing filter is not applied to the velocity fields. 72

Figure 6-4 Dissipation rate spectra corresponding to the high (a) and low (b) swirl flow conditions. Dissipation rate values increase near the resolution limit when a $3 \times 3$ smoothing filter is not applied to the velocity fields. 73

Figure 6-5 Setup for the fixed-displacement experiments. Legend: M: HR 532 mirror, O: light sheet optics, W: piston window, C: calcite crystal, F: narrow-band filter, L:camera lens 74

Figure 6-6 Spectra obtained from band-pass filtering the artificially generated flow field. The values were calculated with the same routine used to obtain the kinetic energy spectrum of the engine flows. The peak occurs at $300 \mu \mathrm{m}$.............. 76

Figure 7-1 Experimental setup for the dual-laser single-camera high speed UV PIV setup. Legend:-M1, M2: HR 355 HT 532 mirrors, W: 1/2 waveplate, PBS: polarizing beam-splitting cube, BD: beam dump, A: aperture, DL: diverging lens, FO: focusing optics, G: gate, M3: $45^{\circ} \mathrm{UV}$-enhanced mirror, ND: neutral density filters, P: glass plate, M4: HR 355 mirror. Dotted arrows indicate polarization of the output beams 81

Figure 7-2 Transmissivity of the 355 narrow-band filter used in the single-laser, singlecamera high-speed PIV setup, showing that the incoming signal is attenuated by approximately $70 \%$ at $355 \mathrm{~nm}$. 83

Figure 7-3 Transmission curve for to the $45^{\circ}$ UV-coated mirror, showing (see also inset with expanded ordinate) between $98 \%$ and $99 \%$ reflectivity at $355 \mathrm{~nm} . \ldots 83$

Figure 7-4 Top view of the engine head and piston bowl showing the location of the field of view used in the dual-laser, single-camera high-speed UV PIV measurements 84 
Figure 7-5 Main equipment and signals used to synchronize the high-speed lasers and cameras used in the UV PIV experiment. Signals are shown in dotted-outline text boxes.

Figure 7-6 Triggering schematic for the dual-laser, single-camera UV PIV experiment 88

Figure 7-7 Average Q-factor and percentage of first choice vectors for the motored and fired engine experiments.

Figure 7-8 Instantaneous flow fields at $61{ }^{\circ}$ BTDC for motored and fired experiments... 95

Figure 7-9 Instantaneous flow fields at $57^{\circ}$ BTDC for motored and fired experiments... 96

Figure 7-10 Ensemble average flow fields at $57{ }^{\circ}$ BTDC for motored and fired experiments.

Figure 7-11 Flow fields at $47^{\circ} \mathrm{BTDC}$ and $45^{\circ} \mathrm{BTDC}$, during the arrival of the spray jets to the field of view. 98

Figure 7-12 Conditions at $37^{\circ} \mathrm{BTDC}$ and $35^{\circ} \mathrm{BTDC}$ for motored (left column) and fired (right column) experiments. 99

Figure 7-13 Instantaneous flow images between $33^{\circ} \mathrm{BTDC}$ and $27^{\circ} \mathrm{BTDC}$ for a single engine cycle. The spark luminosity is captured at $33{ }^{\circ} \mathrm{BTDC}$. The deformation of the plasma channel can be seen at $29{ }^{\circ}$ BTDC and $27{ }^{\circ}$ BTDC. 101

Figure 7-14 Deformation of the plasma channel at $31^{\circ} \mathrm{BTDC}$ and $27^{\circ} \mathrm{BTDC}$ 102

Figure 7-15 Kinetic energy in a $2 \mathrm{~mm}^{2}$ region near the spark plug for the motored-engine experiment. Legend: x-instantaneous values, a ensemble mean kinetic energy values. 103

Figure 7-16 Kinetic energy in a $2 \mathrm{~mm}^{2}$ region near the spark plug for the fired-engine experiment. Legend: x-instantaneous values, - ensemble mean kinetic energy values. 104

Figure 7-17 Kinetic energy of the ensemble mean averaged over 300 cycles in a $2 \mathrm{~mm}^{2}$ region near the spark plug for the motored-engine experiment. 105 


\section{LIST OF TABLES}

Table 3-1 Optical engine specifications. In all cases TDC refers to TDC compression . 22

Table 3-2 Experimental PIV parameters for the preliminary experiment. ...................... 26

Table 3-3 Test matrix for preliminary PIV experiments in the optical engine................ 28

Table 3-4 Post-processing results for preliminary PIV data ....................................... 30

Table 4-1 Parameters corresponding to the single-laser, single-camera high-speed UV

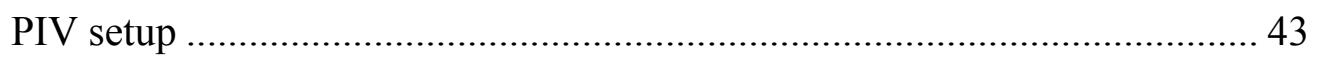

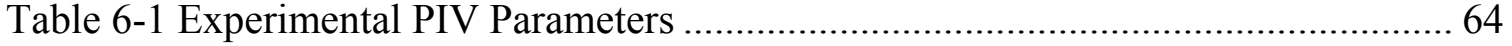

Table 7-1 Experimental parameters for the high-speed dual-laser UV PIV setup .......... 89 


\begin{abstract}
The in-cylinder flow is one of the most influential physical processes in internal combustion (IC) engine operation. It impacts fuel-air mixing, combustion and pollutant emissions. Many flow-related processes occur on a sub-millisecond time-scale. Timeresolved multidimensional flow-velocity data can provide valuable insight into processes relevant to IC engine performance.

This document describes the development of a high-speed particle image velocimetry (PIV) technique using a two-laser, single-camera system and the application to a motored and fired stratified spark-ignition, direct-injection (SIDI) engine. The technique is novel in that it (1) achieves unprecedented temporal resolution: one velocity field at every other crank angle at 2000 RPM for approximately 500 consecutive cycles and (2) uses $355 \mathrm{~nm}$ (UV) excitation wavelength. Advantages include suppressing the detection of combustion luminosity, capturing the luminosity from the spark discharge, and providing the flexibility to integrate planar laser induced fluorescence (PLIF) of biacetyl for simultaneous velocity and equivalence ratio distribution measurements.

Initial PIV experiments conducted in an optical SIDI engine are described and results from the first application of UV PIV in a fired engine at 600 RPM presented. Next, an experiment introducing the simultaneous application of high-speed UV PIV and biacetyl-PLIF is described. The application of UV PIV for velocity measurements in the SIDI engine at $2000 \mathrm{RPM}$ is then presented.

Velocity fields are shown for multiple crank angles of motored and fired engine cycles. The action exerted by the post-injection flow field on the spark plasma channel is captured. Results confirm that the ensemble mean kinetic energy decreases as the piston
\end{abstract}


approaches top-dead-center (TDC) and that, under fired conditions, the flow pattern near TDC is dominated by the residual spray momentum.

The effect of PIV measurement noise on two-dimensional kinetic energy and dissipation rate spectra of IC engine flows is described. Low-resolution $(2 \mathrm{~mm})$ and high-resolution $(0.35 \mathrm{~mm})$ measurements show an abrupt increase in energy and dissipation rate spectra at the spatial resolution limit. This increase can be attributed to noise introduced by the PIV interrogation algorithm. A spatial averaging filter removes the spectral peaks, while attenuating kinetic energy and dissipation rate values near the PIV resolution limit. 


\section{CHAPTER 1}

\section{INTRODUCTION}

\subsection{In-Cylinder Flow in Internal Combustion Engines}

The gas-powered automobile is the most important means of personal transportation for millions of people around the world. For many years, the automobile has impacted the world from socio-economic, political and environmental standpoints. Likewise, current socio-economic, environmental and political conditions continue to influence the development of the automobile. At the heart of the automobile, as we know it today, is the reciprocating internal combustion (IC) engine. While many technological advances have made the IC engine more robust, efficient and powerful, its basic design has remained virtually unchanged throughout the years. Yet, researchers remain fascinated by the processes that occur inside the combustion chamber of an IC engine and continue to both learn from and improve this complex system.

The automotive sector is currently undergoing drastic changes, driven by the need to simultaneously meet increasingly stringent environmental regulations and achieve more efficient operation over a wide range of engine speeds and loads, while satisfying customer demands in terms of performance, safety and reliability. The conventional spark ignition engine is still the most common energy conversion system in the passenger vehicle market. Through the years, its combustion configuration has evolved from carburetion, to throttle-body injection, to port fuel injection (PFI). In today's PFI spark ignition engines, a throttle plate is used to control the amount of air inducted into the combustion chamber. The fuel is injected upstream of the intake valves in appropriate 
amounts to achieve a stoichiometric fuel-air mixture. As a result, these engines provide ample time for the fuel and air to mix, so that at the time of ignition, a stoichiometric and nearly homogenous mixture is present near the spark plug. One of the major limitations of PFI engines is the use of the throttle plate as a method of load control. The throttle plate causes thermodynamic (pumping) losses that degrade thermal efficiency. During cold start, transient film deposits in the intake port cause the engine to require fuel in excess of the stoichiometric amount, which increases fuel consumption and unburned hydrocarbon (UBHC) emissions. These are intrinsic limitations to the PFI engine design. It is unlikely that major innovations in this technology will simultaneously allow further pollutant-emission reductions and fuel economy improvements [1].

Other alternatives such as homogenous charge compression ignition (HCCI) and spark-ignition direct-injection (SIDI) engines are currently the subject of extensive research. The SIDI concept has been revived due to the availability of advanced computer controls and injection systems [1,2]. A SIDI engine can operate in stratified or homogeneous modes. In stratified mode, the load is controlled by injecting the fuel directly into the combustion chamber. A stoichiometric mixture is promoted near the spark plug while maintaining an overall fuel-lean mixture in the rest of the combustion chamber. In this mode, suitable for part load (i.e. city-driving), SIDI engines consume less fuel than conventional PFI engines $[1,2,3]$. Up to $25 \%$ potential fuel economy improvement has been estimated for SIDI engines, depending on the test cycle [1].

Regardless of engine type, turbulence plays a vital role in the processes that govern IC engine cycles. In fact, without turbulence, it would not be possible to drive IC engine-powered vehicles over the broad range of speeds and loads as we do today. A predominant reason is that the laminar burning velocity of commonly used fuels is not high enough to burn the fresh charge during the limited time available for combustion in every engine cycle. As an example, the laminar flame speed of iso-octane (a gasoline component) is near $300 \mathrm{~mm} / \mathrm{s}$ at an equivalence ratio of 1 [4]. At this speed, the flame 
front would travel $86 \mathrm{~mm}$ (a typical stroke length) in $280 \mathrm{~ms}$, which corresponds to 4.5 engine cycles at 2000 RPM. Turbulence wrinkles the flame front, increasing its surface area and the burning rate. This wrinkling effect is governed by the relative magnitudes of turbulence intensity and length scale [5].

In IC engine cycles, the time needed for combustion is usually specified in crank angle degrees (CAD). The combustion duration (in CAD) increases slowly as the engine speed increases [6]. This means that the "real" time (in seconds) available to complete combustion decreases as the engine speed increases. Fortunately, there is a linear increase of turbulence levels with engine speed $[7,8,9]$, which increases the overall burning rate, thereby allowing IC engines to operate over a broad range of speeds and loads.

Also critical to the success of IC engine cycles are several coupled processes involving the in-cylinder flow such as: air-fuel spray interactions, which significantly impact the degree of mixing; interactions of the spark with the in-cylinder flow, which influence ignition source location and stability; and interactions between the in-cylinder flow and the equivalence ratio distribution, which impact the development of the early flame.

There are also unwanted effects associated with in-cylinder mean flow and turbulence. While the stretch induced by velocity gradients is known to increase the flame surface area and burning rate, it has also been shown that highly stretched flames fail to attenuate vorticity by gas expansion. As a result, vortices can weaken the flame to the extinction limit [10]. Research on non-premixed methane-air flames has shown that an increase in the strain rate enhances heat conduction out of the reaction zone. If the strain rate increases excessively, heat production through chemical reaction does not compensate for this heat loss, causing the flame to extinguish [11]. Mean flow effects on the early flame kernel have also been studied. An optimal mean velocity range between 3 $\mathrm{m} / \mathrm{s}$ and $5 \mathrm{~m} / \mathrm{s}$ was identified in the case of premixed combustion [12]. Mean velocities 
below this range increase the residence time of the flame kernel near the spark plug electrodes, where heat loss might lead to its early extinction. On the contrary, mean velocities above this range might convect the flame kernel towards regions of high ignition energy or low temperature (e.g. chamber walls), preventing ignition or leading to quenching of the early flame.

The flow in internal combustion engine is very complex. In-cylinder flows are unsteady due to the reciprocating piston motion. They are also three-dimensional, confined by the cylinder walls and reacting during a portion of the engine cycle. Despite the relatively low Reynolds numbers found in IC engines ( $\operatorname{Re} \sim 1000$ if based on engine bore), resolving the broad range of spatial and temporal flow scales is challenging for both experimental and computational researchers. The complexity of IC engine flows is augmented by inhomogeneity and anisotropy at the large scales, particularly when strategies such as swirl and tumble are employed to increase turbulence intensity late in the compression stroke. The high temperatures and pressures that result from combustion, as well as intricacies in the design of practical combustion chambers, have limited the application of experimental techniques to realistic engine flows.

Cycle-to-cycle variability complicates the fluid mechanics analysis in IC engines. This term is used to describe variations in the time needed to burn the fuel-air mixture from cycle to cycle. Cycle-to-cycle variability causes inconsistencies in the delivered engine power. Further, accounting for its effects often leads to design compromises which can reduce engine power at full load and fuel efficiency at part-load. Understanding the cause of cycle-to-cycle variability and minimizing its effects is of great practical interest.

It has been suggested that fluctuations in the charge velocity near the spark plug play a major role in cyclic combustion variations [13]. These fluctuations have in turn been linked to the flow characteristics at intake-valve-closing. In IC engines, the intake flow discharges as a jet through the intake valve opening. As the inlet jet separates from 
the valve seat, it produces shear layers with large velocity gradients, which create turbulence [6]. Experimental and computational evidence supports that there is substantial dependence of the large-scale cycle-to-cycle variability late in the compression stroke on the design of the intake system [7,14,15]. In [14], highly directed (high swirl) and relatively undirected (low swirl) flows were generated at intake-valveclosing in a two-valve engine with a pancake-shaped combustion chamber. In the high swirl case, the instantaneous velocity fields resembled the mean. The low-swirl flow, on the contrary, exhibited high levels of cycle-to-cycle variability in the large scale-structure near TDC. Due to the great sensitivity of turbulence to initial conditions, differences in the turbulent conditions at intake valve closing can produce significant variability at TDC. Since mixing and flame propagation heavily rely on the flow conditions, these variations cause cycle-to-cycle variability in the burn rate and heat release.

An accurate representation of in-cylinder flows is key to predicting its effects on processes that lead to successful IC engine cycles. The effects just described, however, are difficult to model. Consequently, advances in the development of IC engines and models that reliably capture IC engine processes rely heavily on insights gained through experimental work.

The early motivation for understanding in-cylinder turbulence was detonation (i.e. knock), which occurs when the end gases (i.e. the portion of the charge farthest away from the flame front) auto-ignites before the arrival of the flame. H.R. Ricardo concluded, after numerous experiments conducted during the early 1900s, that the superior efficiency of overhead valve engines could be explained in part due to greater turbulence and shorter flame travel, which made the engine less prone to detonation [16]. Since then, significant research has been devoted to understanding the characteristics, evolution and impact of the in-cylinder flow at various stages in the IC engine cycle. Advancements in this area have contributed to the development of more efficient and less polluting IC engines while meeting market demands around the world. 


\subsection{Experimental Flow Techniques Applied to Internal Combustion Engines}

During the late 1930s, the National Advisory Committee for Aeronautics (NACA) pursued a series of experiments that resemble the basic principle of today's particle image velocimetry (PIV). These experiments were conducted in a single-cylinder fourvalve engine fitted with a glass insert for optical access. Shrouds were used to block a portion of the intake valves, creating different levels of swirl and tumble. Feathers were introduced in the combustion chamber and consecutive images were recorded with a camera at approximately $1700 \mathrm{fps}$. The locations of individual feathers were identified on consecutive images and using the time delay between frames, the velocity of the feathers was calculated. Results from this early work showed that the presence of a shroud, regardless of the configuration used, led to over $50 \%$ increase in turbulent velocity relative to conventional intake valves.

During the mid 1900s, laser Doppler velocimetry (LDV) measurements began to provide new insight on in-cylinder turbulence and cycle-to-cycle variability. It has been found through LDV measurements that the turbulence intensity increases linearly with mean piston speed [7,9] and that under minimum swirl, isotropic conditions (within \pm 20 \%) exist near TDC [7]. LDV measurements have also been used to characterize the evolution of the turbulence intensity during the intake and compression strokes of spark ignition engines [17]. Results show that the intensity of the shear-induced turbulence is greatest during the middle third of the intake stroke, with levels reaching ten times the mean piston speed. Turbulence levels decrease significantly near bottom-dead-center (BDC) as no other source exists after the intake valve closes to sustain it. As the piston moves upward during the compression stroke, only flow structures on the order of the clearance height can survive. At this point and especially for high-tumble flows, the breakdown of larger structures enhances turbulence levels near TDC. 
The selection of a specific experimental technique depends on the problem under investigation. Laser Doppler Velocimetry (LDV) provides valuable velocity information at selective spatial locations as a function of time, which might be sufficient for some diagnostic applications. The development of planar particle image velocimetry (PIV) has enabled the computation of instantaneous flow velocities over larger, two dimensional regions in the spatial domain $[14,35]$. Alone, this information is extremely valuable, but what makes PIV very attractive is that it provides the means to compute derivative quantities, (e.g. strain rates and vorticity components) $[10,19,43]$, which are generally not available through LDV measurements. These gradient-based quantities (e.g. strain rate and vorticity) are involved in energy production, dissipation, and in the mechanisms responsible for energy transfer down to the smallest scales in the energy cascade [18]. Specifically, four components of the instantaneous-velocity gradient tensor can be extracted from planar PIV data. These can be used to gain insight on the spectrum of turbulence throughout the IC engine cycle, which is highly desirable to support the development of advanced combustion strategies and validate turbulence models. In addition, velocity gradient tensor data obtained through planar PIV can be used to calculate four strain rate tensor components and one vorticity component. As described in the previous section, these quantities provide physical insight into processes relevant to internal combustion engine operation.

Reuss, Adrian, et al. first applied the principles of PIV to a motored, four-stroke IC engine in the late 1980s and obtained two-dimensional, instantaneous velocity data. Instantaneous vorticity and strain rate fields were calculated from velocity fields with resolution on the order of $1 \mathrm{~mm}$. Eddy-like vortical structures were identified by spatially filtering the velocity field. This work also showed preliminary images illustrating the use of PIV to obtain gas velocities ahead of the flame front [19]. Subsequent work involving velocimetry measurements in a four-stroke IC engine using PIV provided flow velocities in the burned and unburned gas regions of a firing IC 
engine. The engine was fueled with propane at an equivalence ratio of 0.95 [20]. The two-dimensional kinetic energy, vorticity $\left(\omega_{z}\right)$, and normal strain rate $\left(e_{11}+e_{22}\right)$ fields were computed. Conditionally sampled probability density functions (PDFs) of vorticity and strain rate for the burned and unburned regions were used in the analysis of turbulence-flame interactions.

For many years, the analysis of PIV-based engine data has relied on the use of ensemble statistics through Reynolds decomposition of the velocity fields. This approach stems from the non-stationary nature of turbulent flow in IC engines. That is, the reciprocating piston motion induces a temporally changing mean flow throughout the cycle, so that the flow conditions are statistically different from one crank angle to the next. As a result, ensemble averaging as has been traditionally used in IC engines as a starting point for computing turbulence statistics. This is accomplished by recording data for a series of consecutive or non-consecutive cycles at a given piston location (crank angle). At each crank angle, the instantaneous velocity field is then decomposed as the sum of its mean and turbulent components Equation (1-1). In this equation, $<U>$ represents the ensemble mean and $U^{\prime}$ represents velocity fluctuations about the mean.

$U=<U>+U^{\prime}$

Here, $U^{\prime}$ is referred to as velocity fluctuations as opposed to turbulent fluctuations because it has been debated whether the velocity fluctuations calculated via Reynoldsdecomposition yield an accurate representation of IC engine turbulence. It has been proposed that the method overestimates turbulence levels [7], as the fluctuations from the ensemble mean include both the turbulence and cycle-to-cycle variability of individual cycle means. New approaches to decomposing velocity fields to isolate cycle-to-cycle variability from the turbulence have been suggested [14,21,22], including proper orthogonal decomposition (POD) and temporal filtering of time-resolved velocity data. Nevertheless, Reynolds decomposition remains the most common method for velocity 
data reduction. One notable reason is that most computational fluid dynamics (CFD) software used in the research and development of IC engines are based on this approach. Consequently, experimental flow data acquired in support of development and validation of most engine CFD models are compared on the basis of Reynolds decomposition. While results from ensemble-averaged simulations can be useful in identifying general trends to guide engine design, ensemble averages are not sufficient to investigate cyclespecific events (e.g. misfires), which are influenced by variability in parameters critical to the combustion event from one cycle to the next.

Efforts to diagnose and resolve such problems rely to a great extent on experimental work. Diagnostic techniques must accomplish temporal resolution better than one image per cycle in order to investigate how quantities relevant to combustion evolve during individual cycles. Higher temporal resolution is also needed to increase the probability of capturing random events. Misfires, which have been challenging in stratified SIDI engines, are a practical example of a random event for which high-speed diagnostics can be useful. In stratified SIDI engines, the events that begin with fuel injection and lead to successful flame development, or to a misfire, occur on a submillisecond time scale. If the diagnostic technique only allows data recording at a single crank angle per cycle, the selected crank angle might not coincide with the misfire. The experiment could be repeated many times, each time at a different crank angle, but as previously stated, no two engine cycles are the same. Consequently, the evolution of critical variables leading to the abnormal cycle/event could not be resolved. Increasing the temporal resolution and the number of consecutive cycles over which data are recorded also increases the probability of capturing a random event such as an engine misfire.

The flow inside the combustion chamber plays a substantial role in controlling the combustion process, but its effect is not isolated from other parameters. Coupled interactions of the flow and the mixture composition play a significant role in the success 
of the combustion process. In premixed combustion, the equivalence ratio distribution is nearly homogeneous, but this is not the case in stratified SIDI engines. Since flame propagation depends strongly on the equivalence ratio, the flame, in its early stages of development, might be more sensitive to the local flow conditions in stratified combustion rather than in premixed combustion. Furthermore, lean and rich flames are more susceptible to extinction due to strain [23]. High strain rates at the time of ignition, coupled with fuel-rich or fuel-lean areas around the spark, can lead to suboptimal conditions for early flame development. In view of this, studying complex problems (e.g. misfires) requires the combination of cycle-resolved diagnostic techniques to simultaneously and instantaneously capture parameters relevant to the success of the combustion event, such as the in-cylinder flow and the spatial distribution of the mixture composition.

A limited number of published works on the development and application of highspeed PIV techniques are available. Reeves et al. [24] developed a system consisting of a copper vapor laser (Oxford Lasers LS20) that supplied 30 ns pulses with energies of up to $6 \mathrm{~mJ}$ per pulse. This system also included a Kodak 4540 camera, triggered at $9 \mathrm{kHz}$ to record the Mie scattered signal from acrylonitrile microballoons particles $35 \mu \mathrm{m}$ in diameter. The in-plane spatial resolution of $5 \mathrm{~mm}$ was limited by the camera image format $(256 \times 128$ at $9 \mathrm{kHz})$ and the magnification. Each velocity field consisted of a 16 $\mathrm{x} 16$ matrix of vectors and up to nine full, consecutive cycles could be recorded with this setup.

Following this work, Towers and Towers [21] used a similar system but increased the camera frequency to $13.5 \mathrm{kHz}$, which reduced the effective recording area to $128 \mathrm{x}$ 128 pixels. The triggering system was such that a laser pulse arrived at the beginning of each camera frame, with a time separation equal to the camera inter-frame time of $\sim 74$ $\mu$ s. A final matrix of $15 \times 15$ vectors was obtained with $2.9 \mathrm{~mm}$ spatial resolution. The 
total run time of the engine was limited to $20 \mathrm{~s}$, driven by concerns of damaging the optical cylinder largely due to the accumulation of the seeding particles in the ring pack.

In these two approaches, the use of a single laser operated at a fixed frequency prohibited the flexibility of adjusting the time delay between laser pulses. As the particle displacement between recordings is a critical parameter to the quality of PIV data, it is important that the laser frequency be matched to the speed of the in-cylinder flow, which will vary based on engine design. A more flexible approach was developed by Ghandhi et al. [25], who obtained velocimetry data in an IC engine at $1 \mathrm{kHz}$ with the purpose of investigating the effects of temporal and spatial filtering of the velocity field for turbulence analysis. Data were recorded on the swirl plane of a research engine motored at 600 RPM and 1200 RPM. The high-speed PIV system consisted of frame-straddling CMOS camera (TSI HS2000) and a dual-head Nd:YLF laser that supplied a maximum of $12 \mathrm{~mJ}$ at $527 \mathrm{~nm}$ and $2 \mathrm{kHz}$. Also recently, Jarvis et al. [22] developed a high-speed PIV technique using a dual-head Nd:YLF (Newwave Pegasus) laser synchronized with a CMOS (TSI Powerview HS3000) camera, triggered at $10 \mathrm{kHz}$. At this frequency, the available camera image format was 512 x 512 pixels. The laser output at $527 \mathrm{~nm}$ was 10 $\mathrm{mJ}$ of energy per pulse when operated at $2 \mathrm{kHz}(1 \mathrm{kHz}$ per laser head). The $1 \mathrm{~mm}$-thick light sheet illuminated a $17.5 \mathrm{~mm}^{2}$ area on the tumble plane. In [22] the engine was operated at 1500 RPM and the system was triggered such as to obtain velocity fields at 1.8 crank-angle intervals. The spatial resolution was improved to approximately $1.2 \mathrm{~mm}$. This work focused on isolating cycle-to-cycle variability using time-resolved data.

\subsection{Overview of the Present Work}

This present document describes the design and development of a high-speed particle image velocimetry (PIV) technique that uses $355 \mathrm{~nm}$ excitation light and its application to a stratified spark-ignition, direct-injection engine to capture instantaneous 
velocity data during individual engine cycles and for hundreds of consecutive cycles. Following a practical need for this technique, specific metrics were kept in mind through the entire design and development process. At each step, the design was tested and improved accordingly to meet the following requirements:

1. Achieve a level of temporal resolution that allows for obtaining velocity data at multiple crank angles during individual cycles over multiple consecutive cycles.

2. Apply the technique to a production-type stratified SIDI engine.

3. Provide flexibility for combining the technique with high-speed planar induced fluorescence (PLIF) diagnostics, which would allow for simultaneous acquisition of flow and fuel-air mixture distribution data.

As previously mentioned, high temporal resolution facilitates investigations of how the in-cylinder flow characteristics evolve over individual cycles and the capture of random abnormal events, such as misfires. The second requirement stems from both the need for velocity data in stratified SIDI engines and from the occurrence of abnormal events in a small number of cycles. It is worth noting that the use of this technique is by no means restricted to IC engines. Keeping the end application in mind is useful, however, because engines introduce specific constraints and limitations. Finally, the third constraint recognizes the importance of other parameters, in particular the local mixture composition, to the success of IC engine cycles and strives to facilitate the combination of high speed diagnostic techniques to target problems of interest.

Two features common to all previous high-speed PIV techniques are the use of laser output wavelengths in the green range of the spectrum $(510 \mathrm{~nm}, 527 \mathrm{~nm}$ or $528 \mathrm{~nm})$ and the application of PIV under motored-only conditions. In regards to optical measurements, two drawbacks of firing IC engines are (1) limited run-time before the quartz cylinder temperature reaches the maximum allowable value and (2) detection of soot luminosity in fuel-rich operating conditions. Soot luminosity overlaps with the scatter from the PIV tracer particles when $532 \mathrm{~nm}$ laser light is used for excitation and 
will be detected with the double-frame CCD cameras typically used in PIV applications. To conduct flow-related studies and diagnose random events such as misfires, however, it is important that the engine be run under realistic conditions and this includes igniting the fuel-air mixture over consecutive cycles. The main motivation for the present technique is to fulfill these requirements while providing temporal resolution comparable to or better than what has been currently achieved at 2000 RPM engine speed.

\subsection{Dissertation Outline}

Chapter 2 highlights the most relevant experimental errors associated with PIV measurements in IC engines. General procedures followed throughout this work for optimizing important parameters and minimizing experimental uncertainty are also described in this chapter. Working towards the development and application of the highspeed UV PIV technique, preliminary experiments were conducted in a four-stroke, fourvalve single-cylinder optical SIDI engine to assess the feasibility of applying PIV under motored and fired conditions. These experiments are described and results presented in Chapter 3. Chapter 4 builds on these findings and presents the development and results from the first attempt at developing the high speed PIV technique using $355 \mathrm{~nm}$ laser light. This approach was based on a single-laser, single-camera setup. The single, fixed laser frequency of $16 \mathrm{kHz}$ limited the application of this technique to $600 \mathrm{RPM}$. This is similar to the work described in $[21,24]$, but with the fundamental difference of using UV (355 nm) light. The successful application of UV PIV under these conditions was a significant step towards achieving the end objective. Chapter 5 describes an experiment conducted to assess the feasibility of combining high-speed UV PIV with high-speed planar induced fluorescence (PLIF) of biacetyl to simultaneously image the flow and the equivalence ratio distribution. Chapter 6 presents findings on the effect of PIV measurement errors on the kinetic energy and dissipation spectra of IC engine flows. The 
final high-speed UV PIV technique is described in Chapter 7. An account of the necessary modifications to the single laser-camera system, the application of the technique to the optical SIDI engine and representative results are presented. Conclusions and thoughts for future work are provided in Chapter 8. 


\section{CHAPTER 2}

\section{SYSTEMATIC AND RANDOM ERRORS OF PIV MEASUREMENTS IN}

\section{INTERNAL COMBUSTION ENGINES}

\subsection{Particle Image Velocimetry}

Among the advantages of using particle image velocimetry (PIV) to study IC engine flows, are the non-intrusive, instantaneous character of the measurements, access to two-dimensional velocity data to calculate gradient-based quantities and the possibility to visualize the structure of the flow in the combustion chamber. The basis of PIV can be summarized as follows: the flow under study is seeded with tracer particles carefully chosen such that they faithfully follow the fluid motion [26]. The tracer particles are illuminated with light from a light source (e.g. laser) and the Mie scatter is recorded as images separated by a temporal delay. Either photographic or digital media can be used in the recordings, the latter offering advantages in terms of processing time and temporal resolution, whereas photographic media remains unsurpassed in terms of spatial resolution.

To process the raw PIV data, each image is divided into sub-areas called interrogation areas (spots). Autocorrelation or cross-correlation techniques are applied to determine the most probable displacement of the particles in the interrogation spots. This means that a single velocity vector constitutes an average over a subset of the spatial domain, since an average particle displacement is calculated in the interrogation window. 
The velocity is then calculated using the time delay between illuminations and the magnification at the time of recording.

\subsection{Errors in Digital PIV}

In the last twenty years, considerable research has been devoted to identifying and optimizing experimental parameters critical to obtaining reliable PIV data $[26,27,28,29,31,46]$. . Significant effort has also been devoted to improving particledetection and correlation algorithms. Some of the most critical experimental parameters include the seeding material, size, density and homogeneity, light sheet thickness and uniformity and the time interval between laser pulses. If not properly selected, these parameters can introduce significant errors in the calculated velocity field and velocityrelated quantities.

Even under optimum experimental conditions, both systematic (bias) and random errors are introduced in the PIV measurement. PIV relies on detecting particle motion to calculate flow velocities. It is important then, that the tracer is chosen with the correct size and kinematic viscosity to ensure adequate particle response to the highest turbulent frequencies of interest. If these criteria are met, then the bias error can be defined as the uncertainty associated with estimating the most probable particle displacement. Both the experimental setup and PIV evaluation algorithm can contribute to this uncertainty.

Systematic errors in digital PIV arise when calculated particle displacements tend towards (1) smaller values and (2) integer values. Biasing of the velocity towards smaller values has been attributed to large particle displacements, which might lead to loss of inplane particle pairs. These large particle displacements can in turn be caused by the presence of strong velocity gradients. This bias error has been estimated as 0.1 pixels in a 32 × 32 pixel interrogation area [27]. The second systematic error listed above also known as peak (pixel) locking effect, has mainly been attributed to unresolved particle 
images. It is largely a consequence of the limited resolving power of digital arrays compared to their film counterparts. The resolution of digital arrays is dictated by the spacing between pixels, $d_{R}$. According to the Nyquist criterion, in order to avoid under sampling the raw data, the diameter of imaged tracer particles $d_{e}$ should be at least twice the medium resolution $d_{R}$ (Equation (2-1)). When this criterion is satisfied, the accuracy in the measured particle displacement is negligibly affected by the finite resolution of the recording medium. A spatial offset between interrogation windows can also be used to avoid pixel-locking effects [28].

$d_{e} \geq c_{R} * d_{R}$ and $1 \leq c_{R} \leq 3$

Random errors can be quantified as the RMS deviation in the calculated mean particle displacements. In PIV, random errors arise from non-ideal seeding, non-uniform illumination of the measurement plane, noise in the light field and shot noise in the photo detector, as well as from aberrations caused by imaging with non-ideal optical components. These factors cause irregular particle images, creating uncertainties in the determination of particle centroids.

Based on results from Monte Carlo simulations, Keane and Adrian [29] showed that sufficient particles must be available in the interrogation spot to increase detection probability and the signal of the correlation peak (i.e. SNR). A seeding density of 8-10 particle pairs for a 32 × 32 pixel window was suggested for optimum detection probability. Investigations conducted by Megerle, Sick and Reuss [30] verified these results experimentally. In their work, an artificial displacement field was created using electro-optical image shifting by means of a birefringent (calcite) crystal. Crosspolarized scattered signals from silicon oil droplets approximately $1 \mu \mathrm{m}$ in diameter were evaluated at various f-stops and two magnifications, using settings representative of IC engine setups. Simulating the particle displacement removed uncertainties associated with flow-induced particle motion. This allowed them to isolate and quantify systematic 
and random errors associated with digital PIV recordings and evaluation. Here accuracy was quantified by changes in the ensemble average of the repeatable displacement fields, whereas precision was defined as the RMS of the particle displacements about the ensemble mean. Results demonstrated that RMS variations in the calculated particle displacements are largest when less than five particles are present in a $32 \times 32$ interrogation window and decreased to approximately $2 \%$ of the local average displacement for 8-10 particle pairs. These results were in accordance with previous theoretical investigations [29]. The authors also assessed the influence of parameters such as the magnification, f-stop and focusing on the accuracy and precision of digital PIV measurements. Results revealed that the RMS error increases with decreasing f-stop (increasing apertures). Specifically precision errors of up to $3 \%$ and $1.5 \%$ of the local mean velocity were found for 0.107 and 0.214 magnifications, respectively at f/5.6. These uncertainties were calculated based on images recorded through a flat quartz window.

Full optical access to research engines is generally facilitated by a transparent (quartz) cylinder. The effect of cylinder curvature on the accuracy and precision of digital PIV is an important consideration when assessing the quality of PIV recordings. Following the work just described, experiments were conducted to quantify cylinder curvature effects [31]. This cylinder was built with the same material and dimensions as the one that provides optical access for the present work. Results showed that the accuracy decreased up to $10 \%$ near the wall. Using $\mathrm{f} / 4$ and 0.214 magnification, the precision errors as quantified by the RMS displacement, ranged between $3.5 \%$ and $5 \%$ of the local mean velocity. The noise was found to increase up to a factor of three when compared to experiments conducted with the flat window, mainly due to poor focusing and low light transmission near the wall. These experiments demonstrated that the field of view should be restricted within the center $66 \mathrm{~mm}$ along the $86 \mathrm{~mm}$-diameter cylinder to achieve the least RMS noise and best accuracy. In subsequent experiments conducted 
by Funk [32], the field of view was confined within the center $66 \mathrm{~mm}$ of the optical cylinder. Fixed-displacement experiments using electro-optical image shifting were carried out at 0.68 magnification. The RMS displacement noise was quantified as 0.1 and 0.15 pixels for $\mathrm{f} / 11$ and $\mathrm{f} / 16$, respectively, which corresponds to $1.25 \%$ uncertainty in the measured velocities based on a mean 8 pixel particle shift.

\subsection{Parameter Optimization for PIV Experiments in IC Engines}

While most of the parameters relevant to PIV measurements are selected based on specific experimental requirements, common methods can be used to optimize them. For clarity, these general procedures will be described here and emphasized when needed as the PIV experiments are introduced in subsequent chapters.

The selection of seeding, illumination source, and recording medium determines the flexibility of the PIV setup. Theoretically [40], these parameters are related through Equations (2-2) and (2-3), where $d_{p}$ is the nominal diameter of the tracer particles $d_{e}$ is the particle image diameter, $\delta z$ the depth of field, $\lambda$ the laser wavelength, $M_{o}$ the magnification and $\mathrm{f \#}$ the f-stop of the imaging optics, which is inversely related to the numerical aperture.

$$
\begin{aligned}
& d_{e}=M_{o}\left(1.5 \delta z \lambda+d_{p}^{2}\right)^{1 / 2} \\
& \delta z=4\left(1+M_{o}^{-1}\right)^{2} f \#^{2} \lambda
\end{aligned}
$$

The tracer particle diameter, $d_{p}$, depends on the characteristics of the particle size and distribution from the seeding apparatus. For all the experiments presented here, silicon oil droplets (Dow Corning 510R) of $1 \mu \mathrm{m}$ nominal diameter were used as the tracer particles. The droplets were produced with a six-jet atomizer (TSI-Model 9306).

The small particle size leads to lower signals because the scattering intensity is directly proportional to particle size, but the selection of a non-abrasive material is an important 
consideration when an optical engine is used for the experimental work. Tracer particles must also follow the instantaneous flow motion. The equation describing the motion of a sphere suspended in a fluid can be simplified in cases where the particle density is considerably greater than that of the fluid (i.e. $\rho_{\mathrm{p}} / \rho_{\mathrm{f}}>>1$ ), as it is true for liquid droplets in air. Neglecting shear effects and centrifugal forces, analytical solutions provide a starting point for selecting the appropriate size of tracer particles. The solution corresponding to fully-developed turbulent pipe flow suggests that $1 \mu \mathrm{m}$ water particles in air have $95 \%$ response to turbulent frequencies on the order of $2000 \mathrm{~Hz}$ [33]. Integral length-scales between 1 and $10 \mathrm{~mm}$ have been measured in IC engines with filtering techniques employing cut-off frequencies on the order of $100 \mathrm{~Hz}$ to $500 \mathrm{~Hz}$. This suggests that the response of $1 \mu \mathrm{m}$ tracer particles is sufficient, provided the specific gravity of the tracer particles is near unity. The specific gravity of the silicon oil droplets used in these experiments is listed as 0.988 at $25^{\circ} \mathrm{C}$. Consequently, adequate response to fluid motion is expected.

For each experimental setup, the seeding density was adjusted by controlling the atomizer pressure during engine operation. Using a visualization routine, $32 \times 32$ pixel areas (the final interrogation-spot size) within the field of view were isolated to inspect the number of tracer particles. The seeding density was considered appropriate once 8-10 particle pairs were resolved in the interrogation window [29]. Seeding homogeneity was determined by sampling and comparing different regions in the field-of-view (FOV). Homogeneous seeding distribution was achieved approximately a minute after the tracer particles were introduced through the engine intake system.

Results from Monte Carlo simulations conducted by Keane and Adrian [29] suggest a maximum in-plane particle displacement of no more than a quarter of the final interrogation window size ( $32 \times 32$ pixels). Following this criterion, for all experiments, the laser pulse delay was optimized so that the tracer particles displaced no more than 8 pixels between exposures. This is particularly relevant to the present study due to cycle- 
to-cycle variability of in-cylinder flows. If the laser pulse delay is not optimized, surges in flow velocity from one cycle to the next may cause the loss of in-plane particle pairs. This in turn may lead to poor displacement correlations.

Turbulent flows cover a broad range of spatial and temporal scales. Ideally, the experimental setup would provide sufficient resolution and dynamic range in both space and time to access most of the scales of the turbulent flow field. Since the wavelength is determined by the light source, Equations (2-2) and (2-3) suggest that the magnification $\mathrm{M}_{\mathrm{o}}$ and the f-stop are the two variables that can be adjusted to achieve an appropriate balance of spatial resolution and dynamic range.

Time-resolved measurements in IC engines require laser pulse frequencies in the $\mathrm{kHz}$ range. Since beam output energy decreases with laser pulse frequency, pulse energy is one of the limiting factors in high-speed diagnostics. The amount of light reaching the detector depends on the inverse square of the distance from the object plane. One way to compensate for lower laser energies is to position the imaging system as close to the engine as possible, while keeping in mind field of view and dynamic range requirements. Light collection can also be increased by decreasing the f-stop. As the aperture is increased (by decreasing the f-stop) however, aberrations due to imperfections in the imaging optics introduce experimental errors that are carried through the PIV evaluation routine, ultimately degrading the accuracy and precision of the calculated velocity values [31]. In the experimental setups described throughout this work, the magnification and fstop were selected taking into account both the limitations of the experimental equipment and the requirements of the technique. This follows from the need to optimize the technique as a whole while minimizing experimental uncertainty. 


\section{CHAPTER 3}

\section{PRELIMINARY PIV EXPERIMENTS IN A FIRED SIDI ENGINE}

\subsection{Experimental Equipment and Setup}

The experiments were conducted in a four-stroke, single-cylinder, four-valve, gasoline, spark-ignition, direct-injection (SIDI) engine operated in stratified mode. The engine, described in [34] has a centrally mounted spark plug and a pent roof- shaped combustion chamber. An eight-hole injector $\left(90^{\circ}\right.$ cone angle) is mounted such that part of the fuel is directly aimed at the spark plug, which aids in the fuel-air mixture stratification. Additional engine specifications are provided in Table 3-1.

Table 3-1 Optical engine specifications. In all cases TDC refers to TDC compression

\begin{tabular}{|l|r|}
\hline Connecting Rod & $159 \mathrm{~mm}$ \\
\hline Bore & $86 \mathrm{~mm}$ \\
\hline Stroke & $86 \mathrm{~mm}$ \\
\hline Displacement & $500 \mathrm{~cm}^{3}$ \\
\hline Compression Ratio & $9.5: 1$ \\
\hline Intake Valve Open (IVO) & $362^{\circ} \mathrm{BTDC}$ \\
\hline Intake Valve Close (IVC) & $126^{\circ} \mathrm{BTDC}$ \\
\hline Exhaust Valve Open (EVO) & $139^{\circ} \mathrm{ATDC}$ \\
\hline Exhaust Valve Close (EVO) & $373^{\circ} \mathrm{ATDC}$ \\
\hline Injection Pressure & $10 \mathrm{MPa}$ \\
\hline Start of Injection (SOI) trigger & $57^{\circ} \mathrm{BTDC}$ \\
\hline End of Injection (EOI) trigger & $40^{\circ} \mathrm{BTDC}$ \\
\hline
\end{tabular}



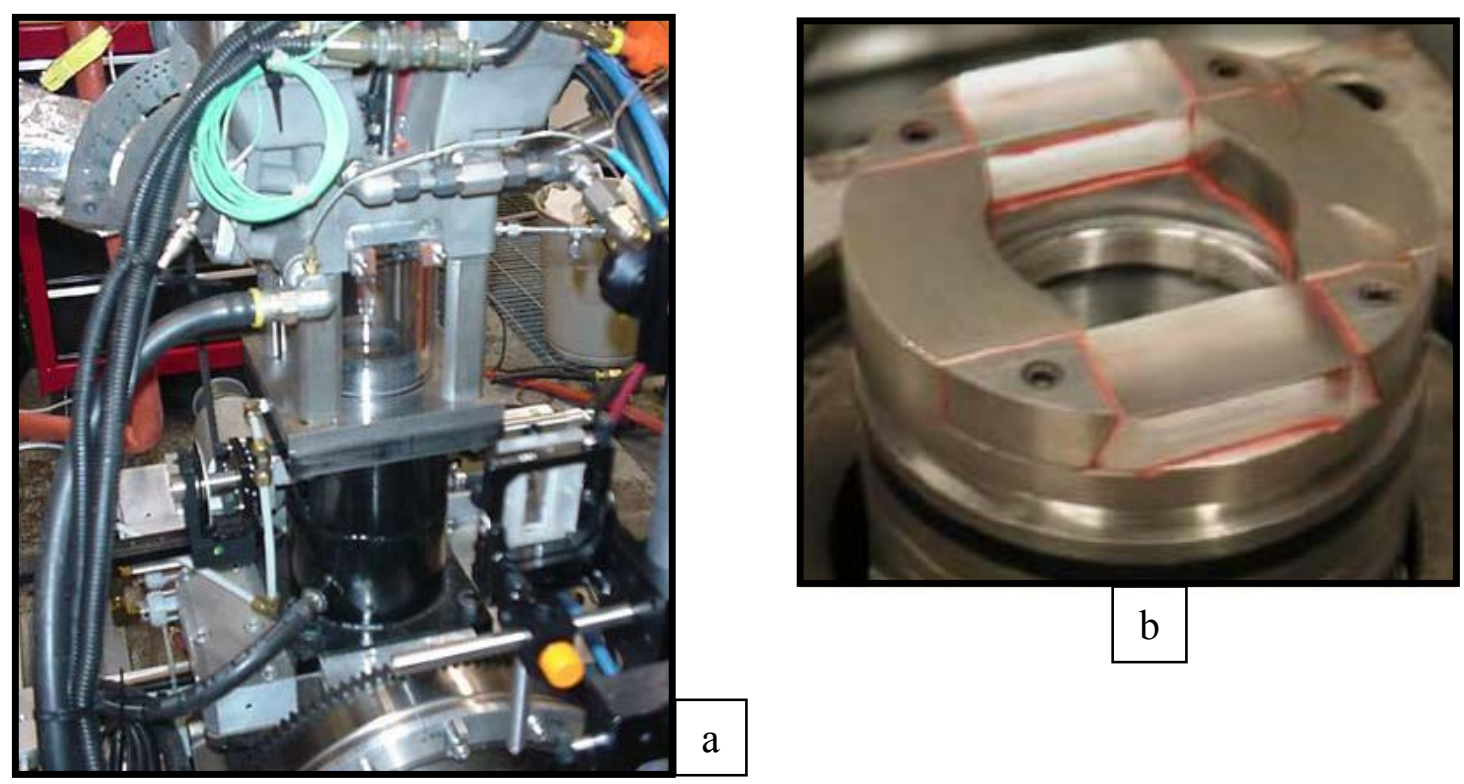

Figure 3-1 Optical engine (a) and piston (b) used in the PIV measurements. The quartz cylinder enables optical access to the engine. The light sheet enters the combustion chamber through the top piston window.

The air mass flow rate is regulated through a pressure valve located upstream of a series of critical orifices. A piezoelectric transducer monitors cylinder pressure. Optical access to this engine is possible through the transparent (quartz) cylinder (Figure 3-1a), through windows located on the ends of the pent roof-combustion chamber, and through windows located on the sides and top of the bowl-in piston (Figure 3-1b).

With the present engine configuration, it was originally unknown whether light scattered off surfaces (e.g. cylinder head and walls) in the proximity of the spark plug (and by the spark plug itself) would overwhelm the signal from the tracer particles, which would prevent obtaining velocity fields near TDC in the areas surrounding the spark plug.

During stratified mode operation, the fuel is injected late in the compression stroke (see Table 3-1). Excessive signal intensity from the residual spray droplets near ignition time could compromise the quality of the measurements. It was also important to identify a crank angle range over which it was feasible to obtain velocity data for three main reasons. (1) As the piston approaches TDC the available imaging area decreases. 
Beyond approximately $20{ }^{\circ} \mathrm{BTDC}$, the spark plug protrudes into the piston bowl. Defocusing of the images due to the relatively thick $(\sim 17 \mathrm{~mm})$ piston windows could prevent further recordings. (2) Change in seeding density. The seeding density in PIV experiments should fall between the laser speckle and particle-tracking modes [35]. In this range, the seeding concentration is high enough that individual particle displacements are difficult to identify, but the particle images do not overlap in the image plane. Since the volume in the combustion chamber progressively decreases as the piston approaches TDC, seeding density requirements are likely change over a broad crank angle range. This is an important consideration given that one of the foremost requirements of the high-speed PIV technique was to obtain velocity data at multiple crank angles over consecutive cycles. (3) Influence of soot luminosity. It was important to determine if, and at which crank angle, the soot luminosity overwhelmed the signal from the tracer particles. A schematic of the setup for these preliminary PIV experiments is provided in Figure 3-2.

Two $532 \mathrm{~nm}$ laser beams of $10 \mathrm{~ns}$ duration exit the cavity of a dual-resonator Nd:YAG laser (New Wave Minilase), providing $13 \mathrm{~mJ}$ of energy per pulse. The beams passed through an aperture and an objective with variable focal length containing two spherical lenses (Rodenstock Modular Focus). A -20 mm cylindrical plano-concave lens was positioned at the exit of the objective to diverge the beams, forming a $1 \mathrm{~mm}$ thick light sheet. The light sheet reflected off a 45 degree turning mirror placed under the extended (Bowditch) piston [36], entering through the quartz piston top to illuminate a plane parallel to the cylinder axis. The imaging area was restricted to $41 \times 86 \mathrm{~mm}^{2}$. 


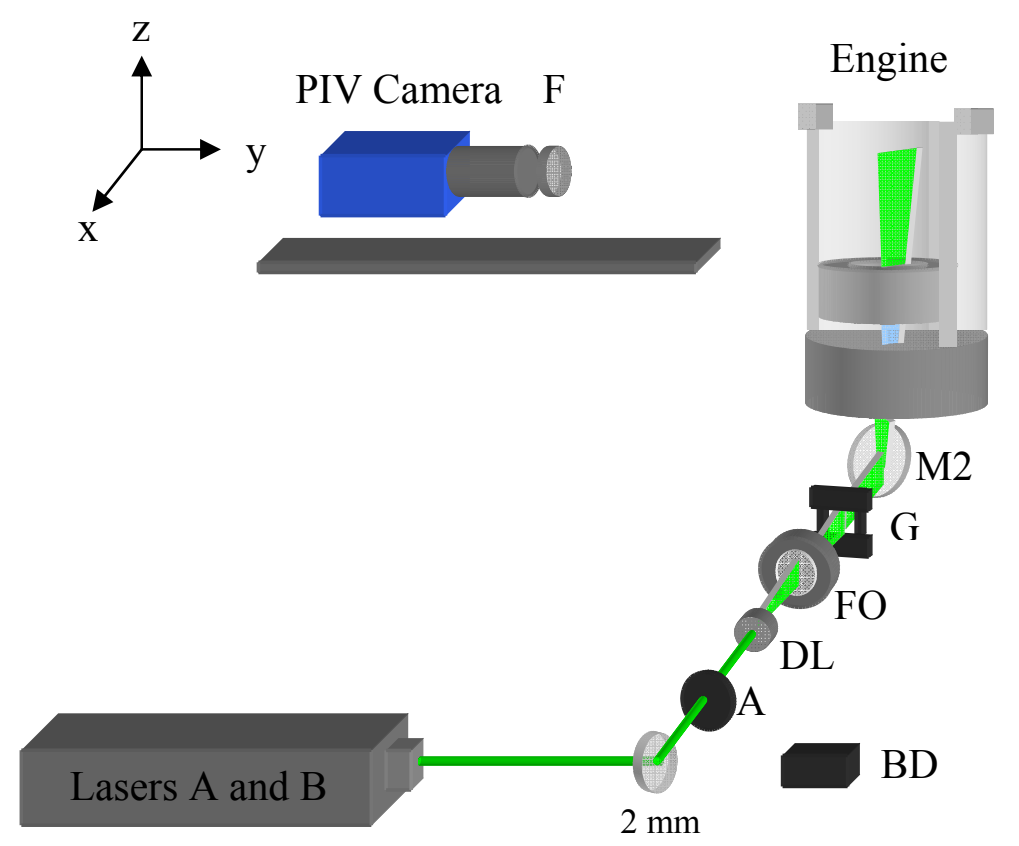

Figure 3-2 Experimental setup for preliminary PIV measurements in the optical engine using $532 \mathrm{~nm}$ wavelength excitation. Legend:-M1: HR 355 HT 532 mirrors, BD: beam dump, A: aperture, DL: diverging lens, FO: focusing optics, G: gate, M2: $45^{\circ}$ turning mirror, F: $532 \mathrm{~nm}$ narrow-band filter.

The tracer particles $(1 \mu \mathrm{m}$ nominal diameter oil droplets) entered the combustion chamber after mixing with the air in the intake plenum. The Mie scattered signals were recorded by a 1376 x 1040 CCD camera (La Vision Imager 3LS-QE) mounted on micrometer-controlled translation stages. The $4 \mathrm{~Hz}$ maximum camera frequency limited the acquisition rate to one image every four cycles at 2000 RPM for the motored runs. A $105 \mathrm{~mm}$ Nikon Micro-Nikkor lens attached to the camera provided adequate focus of the tracer particles. Based on the field of view and 0.126 magnification, approximately 1.5 $\mathrm{mm}$ spatial resolution was achieved. A $532 \mathrm{~nm}$ narrow-band filter was placed in front of the lens to minimize the level of background signal recorded. La Vision Davis 6.2 software was used for equipment synchronization and data processing. A summary of the settings for relevant PIV parameters is provided in Table 3-2. 
Table 3-2 Experimental PIV parameters for the preliminary experiment.

\begin{tabular}{|l|r|}
\hline \multicolumn{1}{|c|}{ Parameter } & Value \\
\hline Magnification, $\mathrm{M}_{\mathrm{o}}$ & 0.126 \\
\hline f-number & 5.6 \\
\hline Depth of field $(\mathrm{mm})$ & 5 \\
\hline Pixel size, $\mathrm{d}_{\mathrm{r}}(\mu \mathrm{m})$ & 6.45 \\
\hline Image size $($ pixels $)$ & 3 \\
\hline Spatial resolution $(\mathrm{mm})$ & 1.5 \\
\hline Dynamic velocity range $(\mathrm{DVR})$ & $50: 1$ \\
\hline Maximum resolvable velocity $(\mathrm{m} / \mathrm{s})$ & 20 \\
\hline
\end{tabular}

Before activating the atomizer, an average of at least 30 images was computed. These background images were then subtracted from the seeded flow images to minimize noise in the raw images, introduced by surface scatter. Additional runs were required to optimize both the seeding density and the delay between laser pulses, based on cylinder volume and engine speed at the time of recording, respectively. Due to engine geometry, the cylinder volume changes more rapidly midway through the compression stroke (around $90^{\circ} \mathrm{BTDC}$ ), than during the second half of the compression stroke (Figure 3-3).

Consequently, a nearly constant seeding setting can be used for crank angles closer to TDC, but the same setting might lead to low seeding densities halfway through the compression stroke. In these experiments the seeding density was optimized separately for $90{ }^{\circ} \mathrm{BTDC}$ and $33{ }^{\circ} \mathrm{BTDC}$. It was found that the setting at $33{ }^{\circ} \mathrm{BTDC}$ worked well for the rest of the crank angles near TDC. 


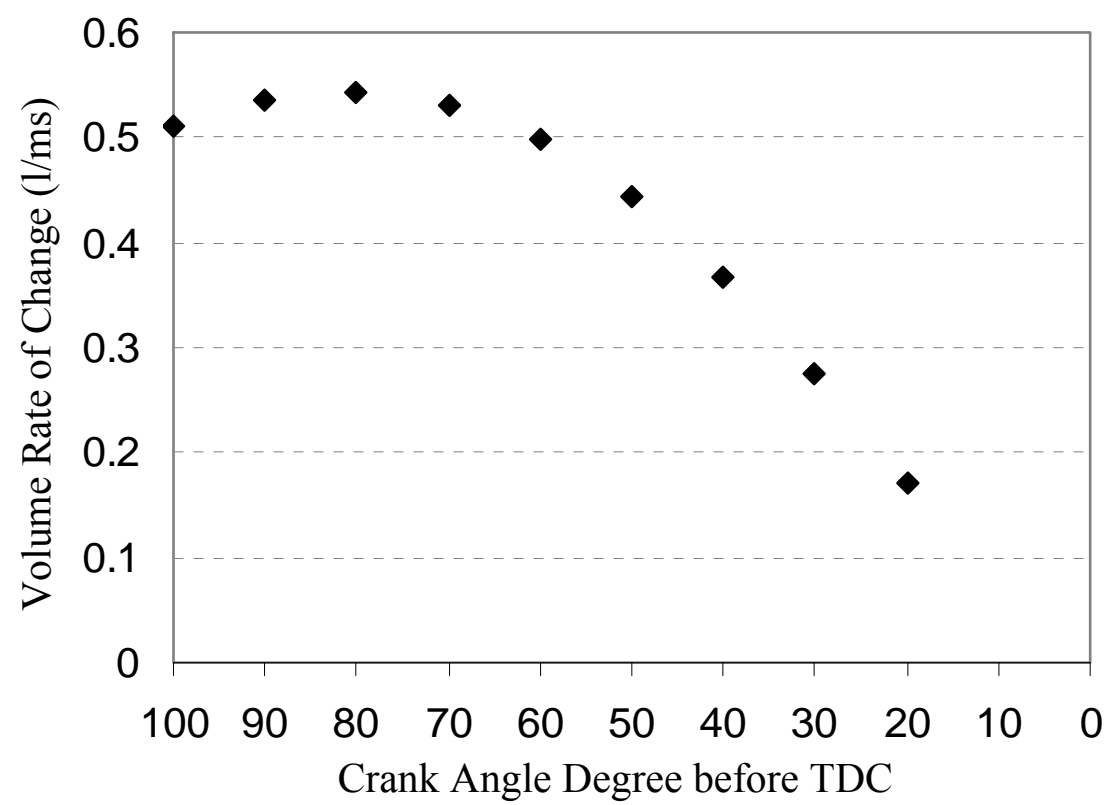

Figure 3-3 Rate of change of the cylinder volume as the piston approaches TDC

Next, a "time sweep" was performed during a single engine run to determine the optimal delay between laser pulses at 600 RPM and 2000 RPM. A set of images was recorded at various time delays. In each case, the instantaneous velocity fields were examined to determine the maximum pixel shift between frames. The last column in Table 3-3 shows that engine speed has the strongest effect on laser-pulse time delay. At 2000 RPM, a $20 \mu$ s delay between laser pulses produced adequate results for all crank angle settings.

The engine was operated at $45{ }^{\circ} \mathrm{C}$ intake temperature and $95 \mathrm{kPa}$ manifold pressure (MAP). A swirl valve located in one of the intake ports can be opened or closed to produce relatively undirected (low swirl) and highly directed (high swirl) intake flows, respectively. In these experiments, the swirl valve remained fully open. Low swirl was selected to test the feasibility of applying PIV under flow conditions that lead to the largest variability at TDC $[7,14]$. This would allow investigating problems of practical interest in stratified SIDI engines, such as misfires. As shown in Table 3-3, seven experiments were conducted in increasing level of complexity. The engine was motored 
at 600 RPM and 2000 RPM for the first two tests. For the rest of the experiments, the engine was fueled with iso-octane and skip-fired (every four cycles) at 2000 RPM. Verifying the feasibility of these experiments at 2000 RPM was of particular importance, since it constitutes a target operating point for SIDI engines. Achieving acceptable temporal resolution at 2000 RPM was a practical design criterion and also a significant challenge for the development of the high-speed UV PIV technique that will be presented in Chapter 7. At 2000 RPM, ignition is triggered at $34^{\circ} \mathrm{BTDC}, 6 \mathrm{CAD}$ after the end of injection. These timings were selected to ensure optimum engine running conditions, defined on the criteria of maximum indicated mean effective pressure (IMEP) and minimum coefficient of variation (COV). In addition, velocity images at crank angles of $33{ }^{\circ} \mathrm{BTDC}$ and beyond are of special interest to investigate the interaction of the flow with the spark plasma channel.

Table 3-3 Test matrix for preliminary PIV experiments in the optical engine.

\begin{tabular}{|c|c|c|c|c|c|}
\hline Test & $\begin{array}{c}\text { Piston Location } \\
(\text { CAD before TDC) }\end{array}$ & $\begin{array}{c}\text { Engine } \\
\text { Speed } \\
(\text { RPM })\end{array}$ & Fuel Injection & Ignition & $\begin{array}{c}\text { Laser pulse } \\
\text { delay }(\mu s)\end{array}$ \\
\hline 1 & 90 & 600 & No & No & 85 \\
\hline 2 & 90 & 2000 & No & No & 20 \\
\hline 3 & 33 & 2000 & Yes & Yes & 20 \\
\hline 4 & 31 & 2000 & Yes & Yes & 20 \\
\hline 5 & 29 & 2000 & Yes & Yes & 18 \\
\hline 6 & 25 & 2000 & Yes & Yes & 18 \\
\hline 7 & 20 & 2000 & Yes & Yes & 18 \\
\hline
\end{tabular}

\subsection{Data Processing}

Velocity fields were calculated within a mask created using the background image. The cylinder head, cylinder walls, and the grafoil linings located in the pent roofcylinder interface were eliminated with this mask to ensure that only seeded areas were 
considered during the interrogation process. In addition to background subtraction, a high-pass filter (10 pixels) was applied to minimize random low frequency fluctuations in the raw images. Velocities were computed by cross-correlating double-frame images, gradually decreasing the interrogation window size $(128 \times 128,64 \times 64$, and $32 \times 32)$ with $50 \%$ overlap. Spurious vectors present in the original vector field were removed by means of a standard deviation operator and replaced by the mean of the adjacent four vectors.

A high-pass filter (20 pixels) was applied to the spray images to minimize low frequency background fluctuations. The spray mask was created using a signal intensity threshold in the vicinity of the spark plug. Only areas with intensities below the threshold were retained for the final mask. Since lower values reduced the region available for velocity field calculations, selection of the optimum threshold was based on the vector density and quality of the resulting velocity fields. Spray and velocity masks were combined to create the final interrogation region. The application of a median filter to remove spurious vectors was more challenging in this case. If too stringent, too many vectors were removed, reducing the final vector density. At the same time, it was desired to eliminate as many spurious vectors to obtain a valid description of the velocity fields. Finally, a $3 \times 3$ pixel top-hat filter was applied to smooth the resulting vector fields.

\subsection{Results}

One of the available metrics to estimate the quality of velocity fields computed with PIV is the percentage of vectors retained after post-processing. Each set was processed a number of times using different median filter parameters. The percentages of first choice vectors shown in Table 3-4 are representative of the data set over a range of filters and provide a measure of the quality of the pre-processed fields. The detectability, 
defined as the ratio of the displacement correlation peak to the noise peak [29], lies between 2 and 2.5 in all cases.

Table 3-4 Post-processing results for preliminary PIV data

\begin{tabular}{|c|c|c|c|c|}
\hline Test & $\begin{array}{c}\text { Piston Location } \\
(\text { CA BTDC })\end{array}$ & \#Images & Motored Only & After Ignition \\
\hline & & & $\% 1^{\text {st }}$ Choice Vectors & $\% 1^{\text {st }}$ Choice Vectors \\
\hline 1 & $90(600$ RPM $)$ & 150 & 98 & NA \\
\hline 2 & 90 & 150 & 98 & NA \\
\hline 3 & 33 & 50 & 93 & 80 \\
\hline 4 & 31 & 30 & 96 & 70 \\
\hline 5 & 29 & 50 & 96 & NA \\
\hline 6 & 25 & 50 & 93 & NA \\
\hline 7 & 20 & 50 & 85 &. \\
\hline
\end{tabular}

With combustion, the optical cylinder is subject to high mechanical and thermal stresses and as a result, motoring is used whenever possible to extend engine life. Since the $90^{\circ} \mathrm{BTDC}$ recordings were obtained during motored-only runs, the highest number of images was also recorded at this crank angle. Engine run-time is less of a problem with high-speed imaging because much larger data samples can be acquired over shorter time periods. Seeding-oil deposition on the cylinder walls toward the end of the experiment required engine disassembling and cleaning after each run.

The first two experiments were mainly focused on verifying the linear scaling of fluid velocity with piston speed. Since this is a well established relation, independent of engine geometries and levels of swirl, it provided a good way to assess the reliability of the data. Instantaneous velocity fields at 600 and 2000 RPM are shown in Figure 3-4. 

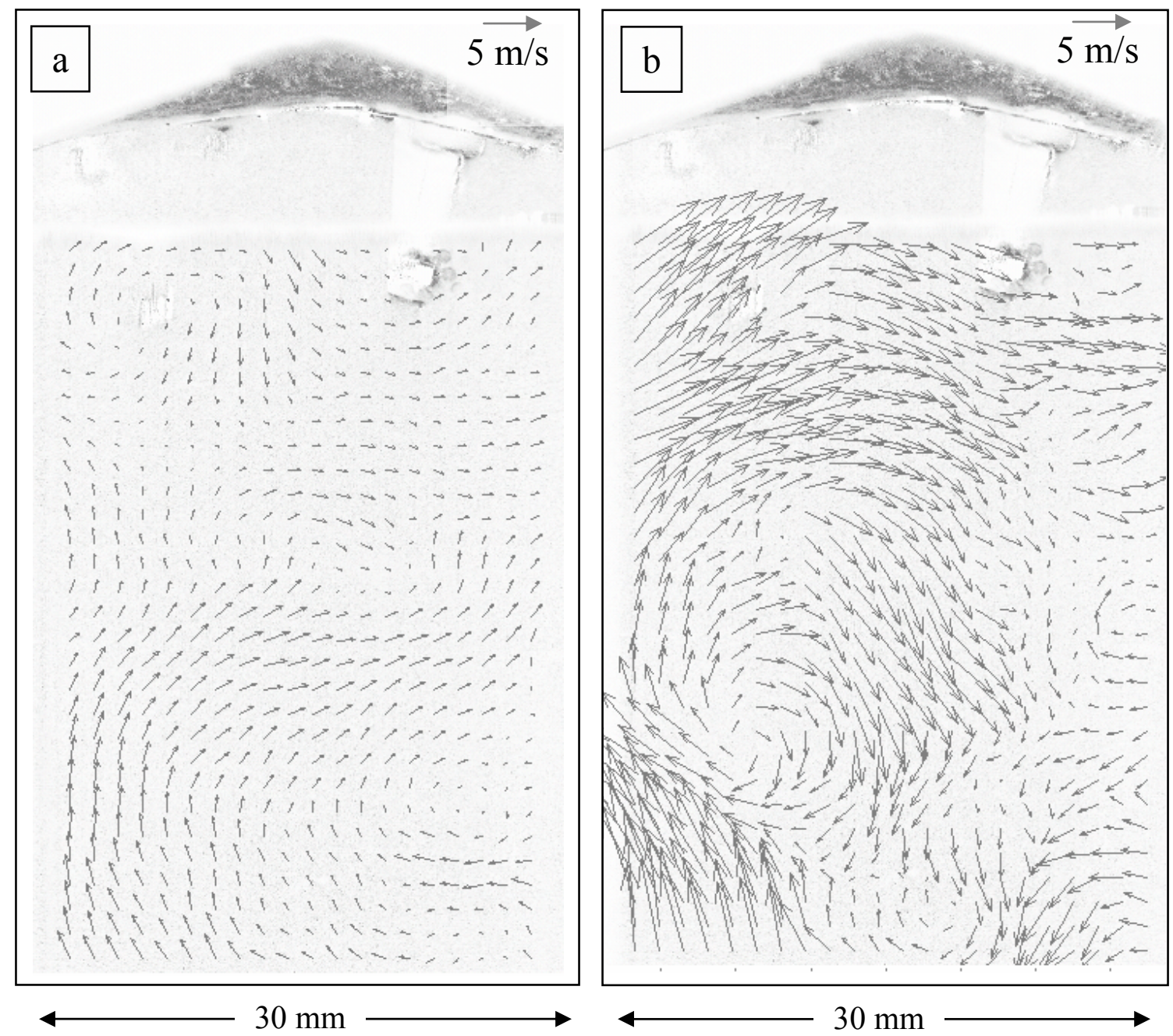

Figure 3-4 Instantaneous velocity fields at 600 RPM (a) and 2000 RPM (b). Both images were recorded at $90^{\circ} \mathrm{BTDC}$. Every other vector is shown.

These velocity fields have been superimposed onto their corresponding background images to show the location of flow structures with respect to important features in the combustion chamber such as the spark plug and the injector. The white stripe near the cylinder head in each image is due to the presence of grafoil linings between the quartz cylinder and cylinder head, which blocks a portion of the field of view. In Figure 3-5, the ensemble average velocity $(<\mathrm{U}>)$, calculated based on 150 cycles, has been normalized by the mean piston speed $\left(U_{\text {piston }}\right), 5.7 \mathrm{~m} / \mathrm{s}$ and $1.7 \mathrm{~m} / \mathrm{s}$ for 2000 RPM and 600 RPM, respectively. The vectors have nearly equal lengths, 
illustrating the linear scaling of mean flow velocity with piston speed, regardless of differences in flow structure details. The normalized values $\left(<\mathrm{U}>/ \mathrm{U}_{\text {piston }}\right)$ averaged over the field of view are $0.75 \mathrm{~m} / \mathrm{s} \pm 0.37 \mathrm{~m} / \mathrm{s}$ and $0.63 \mathrm{~m} / \mathrm{s} \pm 0.37 \mathrm{~m} / \mathrm{s}$ for $600 \mathrm{RPM}$ and 2000 RPM, respectively.
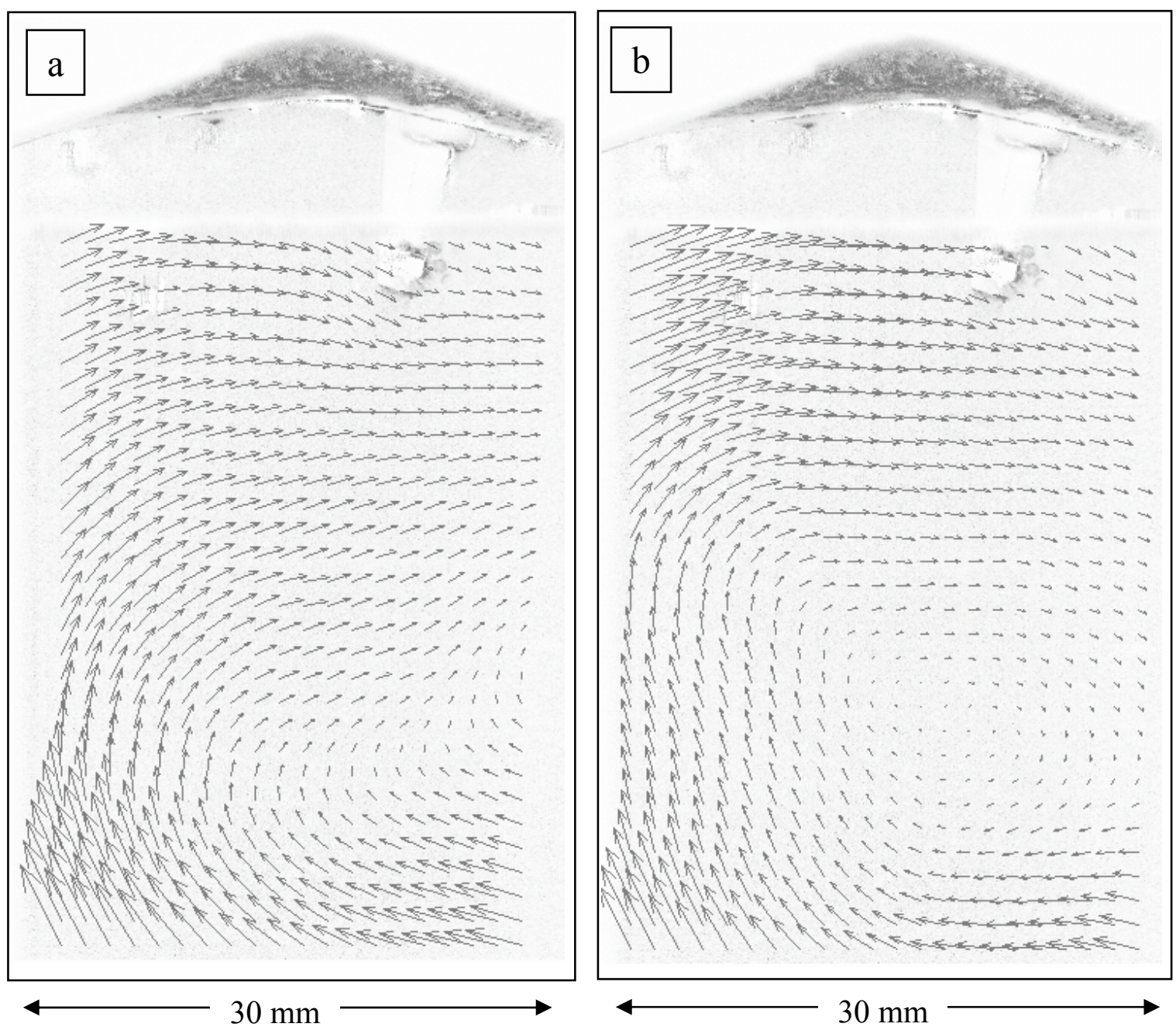

Figure 3-5 Ensemble average flow fields at 600 RPM (a) and 2000 RPM (b) normalized by the mean piston speed. Every other vector is shown.

Examples of individual velocity fields at various crank angles near TDC are shown in Figure 3-6. The left column (images a-d) shows instantaneous velocity fields under motored-only conditions, that is, in the absence of fuel spray or combustion. Flow 
fields computed from PIV images acquired during fired runs are shown on the right column (e-h). In both the motored-only and fired runs, a set of fifty images was recorded while motoring the engine to assess the seeding density and homogeneity level in the absence spray-residual effects. For the fired cases, iso-octane was then injected at 57 ${ }^{\circ}$ BTDC and the ignition driver activated at $34{ }^{\circ}$ BTDC. The first three rows shown in Figure 3-6, from top to bottom, correspond to 33, 31 and $29^{\circ} \mathrm{BTDC}$ for motored (left) and fired (right) runs. While the end-of-injection is expected at $40{ }^{\circ} \mathrm{BTDC}$, significant Mie scatter from the spray residual is present at $33{ }^{\circ} \mathrm{BTDC}$, one crank angle after triggering ignition.

Even though the specific characteristics of the instantaneous flow fields vary from cycle to cycle, all instances show clockwise rotation, consistent with the tumble motion induced at intake-valve-closing. In the motored-only runs, at least one large-scale ( 5 $\mathrm{mm}$ ) vortical structure is captured between the piston top and the cylinder head. The swirl centers however, vary from cycle-to-cycle.

By comparing images $e$ and $f$, it is apparent that the Mie-scatter intensity from residual spray droplets decreases at $31^{\circ} \mathrm{BTDC}$. This is expected, since the images are recorded $9 \mathrm{CAD}$ after the end of injection. The effects of the spray however, can be seen even after injection has stopped. The clockwise charge motion is modified by the residual spray momentum. The spray also causes a velocity increase in the vicinity of the spark plug, which is expected given that for the present SIDI configuration, stratification is achieved by aiming one of the high-pressure spray jets at the spark plug electrode.

At $25{ }^{\circ} \mathrm{BTDC}$, only a velocity field corresponding to a motored-only run is shown because combustion affected the quality of velocity fields computed at this crank angle. The temperatures rose above the boiling point of the oil particles, causing the seeding to become inhomogeneous in the vicinity of the spark plug and preventing the computation of reliable velocity data. The signal from combustion product luminosity, specifically soot luminosity, became overwhelmingly strong at $25^{\circ}$ BTDC (Figure 3-6 (h)). 

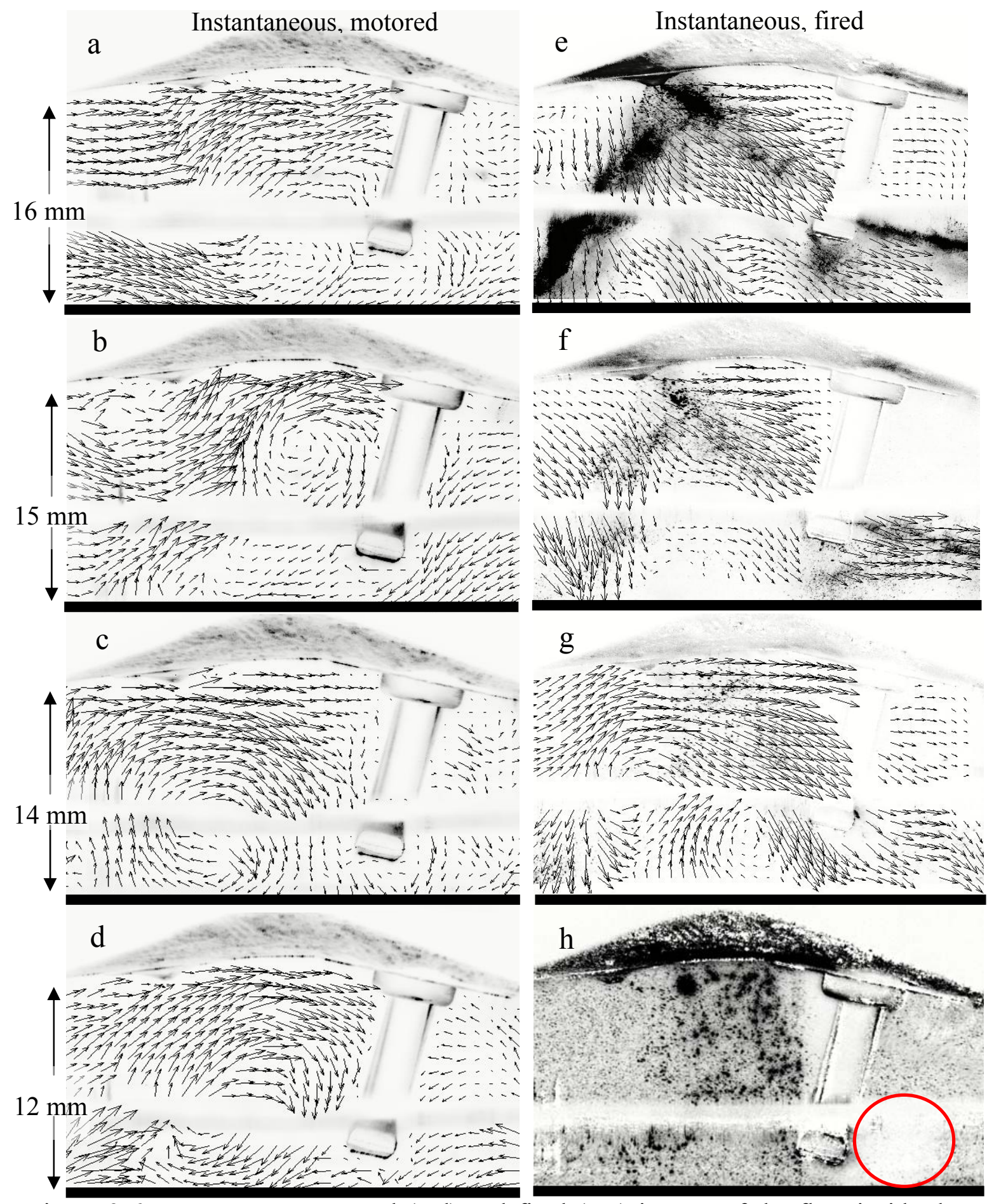

Figure 3-6 Instantaneous motored (a-d) and fired (e-g) images of the flow inside the cylinder of a SIDI engine. Substantial soot luminosity prevented the acquisition of images near the spark plug at $25^{\circ} \mathrm{BTDC}$, as shown by the red circle in (h). 
Results also showed that for each double-frame image recorded at this crank angle, the highest intensities were recorded in the second frame. During double-frame recording, the second frame was exposed for approximately $100 \mathrm{~ms}$, which is orders of magnitude larger than for the first frame. This, coupled with the strong emission of soot in the visible range of the spectrum, caused the recording of unwanted signal and prevented successful cross-correlations at $25^{\circ} \mathrm{BTDC}$.

Although not shown, images were also acquired at $20^{\circ}$ BTDC. In addition to the limitations found at $25^{\circ} \mathrm{BTDC}$, here, the location of the piston top just below the ground strap of the spark plug further reduced the field of view. This would have prevented the computation of velocity data even in the absence of combustion.

It should be emphasized that obtaining velocity data during combustion is not the goal of this work. The silicon oil droplets used as PIV tracers cannot withstand combustion temperatures even if soot luminosity was not a problem. Without the intense soot luminosity however, it was likely that some flow features in the vicinity of the spark plug could be captured. This was a desirable outcome due to the importance of the flow field in near-ignition events in SIDI engines.

It was desirable to test the effect of reducing the exposure time of the second camera frame on the strength of the detected soot-luminosity signal. The duration of the second camera frame, however, could not be reduced due to intrinsic limitations in interframe data transfer speed. Consequently, solving the soot luminosity problem would require a different approach. Since the soot luminosity occurs largely in the visible range of the spectrum, using $355 \mathrm{~nm}$ laser light had the potential to solve this problem. The tracer particles would scatter at $355 \mathrm{~nm}$ and this signal could be spectrally separated from soot luminosity. Further, signals from spark luminosity that fall on the ultraviolet region could be recorded, so that using a combination of high speed imaging and UV excitation could allow imaging the plasma channel. Admittedly, imaging on a single plane would not allow capturing the full three-dimensional motion of the plasma channel. Using UV 
excitation was however, a starting point to partially visualize the dynamics of the plasma channel and its interaction with the flow. Another reason for using UV excitation was the possibility to combine high-speed planar PIV and high-speed PLIF for simultaneous imaging of flow and equivalence ratio distribution near TDC, which is one of the main motivations for this work. The use of biacetyl as a fuel tracer for quantitative

characterization of the equivalence ratio distribution in SIDI engines has been recently documented in the literature $[37,38]$. The main advantage of using biacetyl as a fluorescence tracer is the availability of frequency-tripled Nd:YAG lasers as excitation sources. These lasers can provide pulses in the kilohertz range with acceptable energy output. Fluorescent tracers such as toluene, acetone and 3-pentanone, typically used in IC engine studies have absorption bands near $280 \mathrm{~nm}$ and require $\mathrm{KrFl}, \mathrm{XeCl}$ or frequency-quadrupled Nd:YAG lasers, which provide insufficient pulse energy and/or repetition rates for the current applications.

\subsection{Conclusions}

Preliminary PIV experiments using $532 \mathrm{~nm}$ laser light and data acquisition rates of one image every four engine cycles were conducted in a fired stratified SIDI engine. This work sought the following objectives, (1) assess the feasibility of conducting PIV near TDC in an SIDI engine under both motored-only and fired conditions and (2) identify the limitations of this approach for the future development of high-speed PIV imaging in the same engine.

The most relevant findings were as follows:

a. Vector fields can be obtained during the second half of the compression stroke, from $90{ }^{\circ} \mathrm{BTDC}$ to $25^{\circ} \mathrm{BTDC}$, except during and shortly after fuel injection $\left(57^{\circ} \mathrm{BTDC}\right.$ to $\left.34{ }^{\circ} \mathrm{BTDC}\right)$ due to the limited dynamic range of the PIV setup. 
b. Very intense soot luminosity was recorded at $25^{\circ} \mathrm{BTDC}$, preventing the acquisition of reliable velocity data in the vicinity of the spark plug at this crank angle, and possibly between $29^{\circ} \mathrm{BTDC}$ and $26^{\circ} \mathrm{BTDC}$. Nonetheless, $25^{\circ} \mathrm{BTDC}$ constitutes an upper limit to the crank angle range over which velocity fields can be acquired on the tumble plane.

c. The possibility of eliminating detection of soot luminosity while capturing the spark discharge and to combine PIV with biacetyl PLIF to simultaneously image flow velocity and the equivalence ratio distribution at high temporal resolution drove the selection of $355 \mathrm{~nm}$ (UV) excitation light for the high-speed PIV technique. 


\section{CHAPTER 4}

\section{HIGH-SPEED UV PIV USING A SINGLE LASER-CAMERA SYSTEM}

Having identified $355 \mathrm{~nm}$ light as an important design constraint for the highspeed imaging of the flow inside the SIDI engine, it was important to evaluate the feasibility of the experimental equipment for high-speed UV PIV imaging and to determine the optimum experimental settings.

\subsection{Experimental Equipment and Setup}

The experiments were conducted in the optical SIDI engine described in Chapter 3. Even though the main layout is shown in Figure 3-2, the use of $355 \mathrm{~nm}$ wavelength required important changes in terms of diagnostic equipment and optical components.

A single, frequency-tripled diode-pumped Nd-YAG laser (Quantronix Hawk) was operated at $16 \mathrm{kHz}$. This contrasts the two $10 \mathrm{~Hz}$ lasers used in previous PIV experiments. At $16 \mathrm{kHz}$, the current laser produced consecutive pulses of $100 \mathrm{~ns}$ duration and nominal pulse energy of $0.3 \mathrm{~mJ}$. The drastic decrease in pulse energy, about 40 times less than previously available, results from the frequency-tripling process and from the two orders of magnitude increase in laser frequency. The narrow $(0.7 \mathrm{~mm})$, circular, multi-mode beam was reflected using a HR 355/HT $53245^{\circ}$ turning mirror. The $355 \mathrm{~nm}$ light beam passed through light-sheet forming optics (Rodenstock Modular Focus), and the resulting $1 \mathrm{~mm}$-thick light sheet entered the combustion chamber via a UV-enhanced mirror. Silicone oil droplets of $1 \mu \mathrm{m}$ diameter, produced with a six jet atomizer (TSI Model 9306), were used as scattering particles. Seeding density and homogeneity were 
adjusted according to the procedure described in Section 2.3. The light sheet entered the engine parallel to the tumble plane through the top piston window, illuminating a plane that bisected the injector and the spark plug electrode. Mie scattering signal from the tracer particles was collected through the transparent (quartz) cylinder by a high-speed CMOS camera (Vision Research Phantom 7.1) placed perpendicular to the tumble plane. With this camera, full frame resolution ( $600 \times 800$ pixels) can be achieved up to $4.8 \mathrm{kHz}$. The camera is equipped with 8 GB of onboard memory. Consecutive images can be recorded at the selected rate until the memory is full. Alternatively, the memory-gate function can be enabled to record only during the portion of the cycle of interest and increase the number of consecutive cycles over which data are recorded.

The transmission characteristics of the camera entrance windows were measured to verify that the recorded signal suffered minimal attenuation. As shown in Figure 4-1, the built-in entrance windows allowed approximately $87 \%$ transmissivity at $355 \mathrm{~nm}$.

Since a single, fixed laser frequency was used for these experiments, it was important to select this frequency to ensure an adequate time delay between laser pulses based on engine speed. Since the laser and the camera would be triggered at the same frequency, selecting 2000 RPM as the engine speed would have required the lasers to run at $48 \mathrm{kHz}$. This frequency would have approximated the $20 \mu$ s delay needed at 2000 RPM (refer to Table 3-2). At this frequency, the camera image format would be $256 \mathrm{x}$ 128 , which would reduce the maximum number of vectors to 128 . To compute 128 vectors, the entire recording area would have to be seeded and the light sheet would have to extend throughout the entire FOV. In practice, the number of vectors in the FOV would be less due to the need to mask areas of high scatter (e.g. spark plug location) and the reduced width of the light sheet, selected to maximize signal strength. 


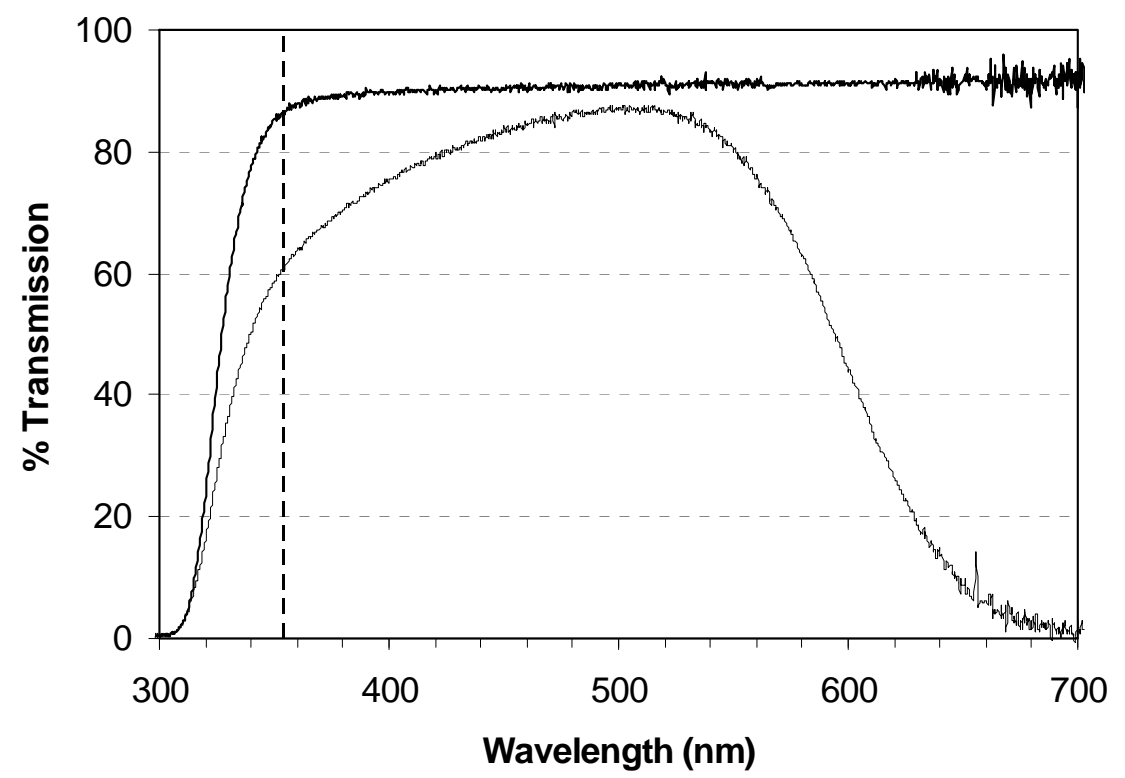

Figure 4-1 Transmissivity of the Phantom V7.1 entrance-windows. The built-in windows, used in the current experiment allow $87 \%$ transmissivity at $355 \mathrm{~nm}$.

Based on these considerations, a 600 RPM engine speed was selected. A starting value for the time delay for 600 RPM was estimated at approximately $60 \mu$ s by linearly scaling the time delay requirement near TDC at 2000 RPM. The closest integer frequency, $16 \mathrm{kHz}$, was then selected. The adequacy of the corresponding $62.5 \mu$ s time delay was then verified by ensuring that the maximum pixel shift between recordings was no more than $1 / 4$ of the final interrogation window size. The $16 \mathrm{kHz}$ temporal resolution requirement was met at the expense of reducing the recording area to $352 \times 352$ pixels. The portion of the engine cycle of interest encompasses the crank angles just prior to injection, through the early stages of flame development. Data recording was externally triggered at $45{ }^{\circ} \mathrm{BTDC}$ and paused at $25^{\circ} \mathrm{BTDC}$ until the next cycle, yielding a total of 8800 images over 100 consecutive cycles. At this rate, approximately four images per crank angle were recorded. Only a small $\left(11 \times 11 \mathrm{~mm}^{2}\right)$ area near the spark plug (Figure 4-2) was investigated for three main reasons, (1) low laser pulse energy at $16 \mathrm{kHz}$ (2) reduced sensitivity of the CMOS camera below $400 \mathrm{~nm}$ and (3) desire to maintain similar spatial resolution $(\sim 1 \mathrm{~mm})$ as for the previous, low-acquisition rate experiments. 


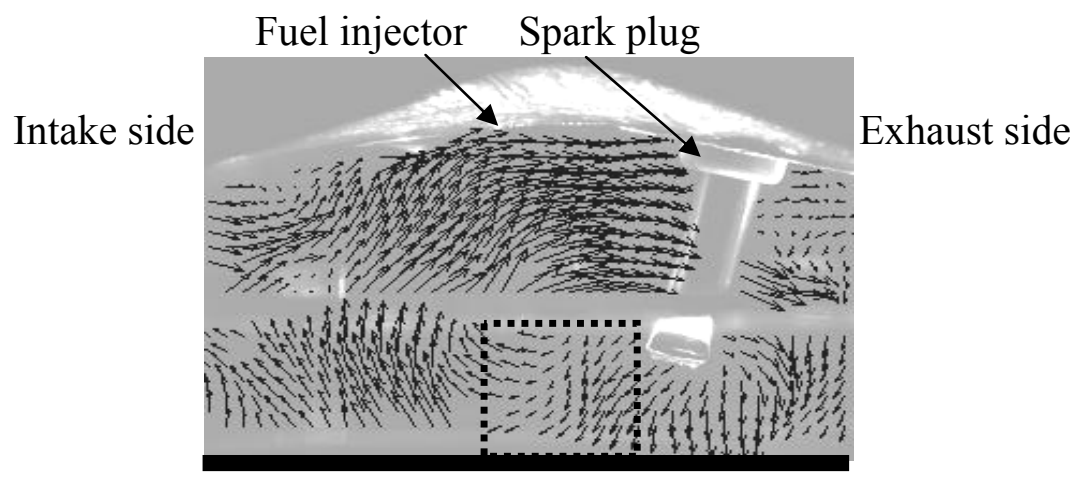

Figure 4-2 Large field-of-view flow characteristics at $34^{\circ}$ BTDC (compression), from experimental results presented in Chapter 3 . The dotted line indicates the location of the $11 \times 11 \mathrm{~mm}^{2}$ imaging area used in the present experiment with respect to the spark plug and injector.

Mie-scattered signals were imaged onto the camera using a $105 \mathrm{~mm} \mathrm{f/4.5} \mathrm{UV} \mathrm{lens}$ (Coastal Optics SLR). A $355 \mathrm{~nm}$ interference filter was placed in front of the lens to minimize background signal recording. An in-plane spatial resolution of $1.2 \mathrm{~mm}$ based on 32 x 32 pixel interrogation window size was achieved with 0.7 magnification. This spatial resolution is sufficient to provide information about the integral scales, which range from 1 to $10 \mathrm{~mm}$ based on previous measurements on the same engine [32]. The fnumber was set at 4.5 to maximize light collection efficiency while maintaining an acceptable depth-of-field. Increasing the magnification, decreasing the f-stop and decreasing the output wavelength from $532 \mathrm{~nm}$ to $355 \mathrm{~nm}$ considerably decreased the depth of field, as suggested by Equations 2-1 and 2-2. This creates additional challenges in achieving consistent and accurate light sheet alignment and overlap from shot to shot, due to engine vibrations. These challenges were essentially eliminated by using a single laser.

Equation (2-3) provides an estimate for the depth of field under the assumption of diffraction-limited optics. To obtain a practical estimate and properly adjust the focus, seeding particles were introduced in the cylinder before running the engine by manually 
opening the intake and exhaust valves. With the camera and laser synchronized, particles images were recorded on sequential frames. Due to cylinder curvature, meridional and sagittal rays follow different optical paths, causing particle-image aberrations. As a result particle images appear vertical and horizontal at the focal positions of the meridional and sagittal rays, respectively [31]. Between these lies the circle of least confusion. At this location particle images appear most round (in focus). Keeping this in mind, the camera position was adjusted using micrometer-driven translation stages while simultaneously recording particle images. The camera location at which the particle image shapes started to appear vertical was noted based on the micrometer reading. The camera was continually translated past the focal plane until the shape of the particles just appeared horizontal. The micrometer reading at this location was noted and subtracted from the starting location. Using this method, the depth of field was estimated as approximately 2 $\mathrm{mm}$. A summary of the PIV parameters just described is provided in Table 4-1. 
Table 4-1 Parameters corresponding to the single-laser, single-camera high-speed UV PIV setup

\begin{tabular}{|l|r|}
\hline \multicolumn{1}{|c|}{ Parameter } & \multicolumn{1}{c|}{ Value } \\
\hline Light sheet width & $5 \mathrm{~mm}$ \\
\hline Light sheet thickness & $1 \mathrm{~mm}$ \\
\hline Field of view & $11 \mathrm{~mm}^{2}$ \\
\hline Magnification & 0.7 \\
\hline f-number & 4.5 \\
\hline Depth of field & $2 \mathrm{~mm}$ \\
\hline In-plane spatial resolution & $1.2 \mathrm{~mm}$ \\
\hline Dynamic velocity range & $63: 1$ \\
\hline Maximum resolvable velocities & $5 \mathrm{~m} / \mathrm{s}$ \\
\hline Engine speed & $600 \mathrm{RPM}$ \\
\hline Intake temperature & $45^{\circ} \mathrm{C}$ \\
\hline Intake pressure & $95 \mathrm{kPa}$ \\
\hline
\end{tabular}

\subsection{Data Processing}

Consecutive images were cross-correlated using a commercial software package (LaVision Davis 7.1) to obtain four instantaneous velocity fields per crank angle. For some of the discussion below, results were grouped into sets that combine all data within one degree crank angle.

A geometric mask was created to exclude areas of high surface scatter. An algorithm was added to the mask to dynamically block areas of suboptimal seeding density (e.g. the highly dense fuel spray). A high-pass filter (15 pixels) was applied to minimize random low frequency background fluctuations in the raw images. The filter size was chosen at least two times the nominal particle image width to retain the signal from the particles. A final interrogation window of $32 \times 32$ pixels with $50 \%$ overlap was used in the cross-correlation algorithm. Spurious vectors present in the original vector field were removed by means of a standard deviation operator and replaced by the mean 
of the adjacent four vectors. The velocity fields were then smoothed using a 3 x 3 pixel top-hat filter to minimize error propagation when computing velocity gradients.

From the two-dimensional velocity field, it is possible to compute two normal $\left(e_{11}\right.$ and $e_{22}$ ) and one shear strain rate component $\left(e_{12}\right)$. The third normal strain rate component $\left(e_{33}\right)$ can be calculated using continuity, under the assumption of small density changes with time (i.e. $\rho^{-1} \partial \rho / \partial t \sim 0$ ). This point will be discussed further in the results section. The strain rate components were computed according to Equation (4-1). Velocity gradients were calculated by taking first order differences of the twodimensional velocity field at each point.

$$
e_{i j}=\frac{1}{2}\left(\frac{\partial u_{i}}{\partial x_{j}}+\frac{\partial u_{j}}{\partial x_{i}}\right)
$$

In addition to the instantaneous image sequences that will be presented and discussed in the next section, a spatial average of each of the four velocity fields available at every crank angle was computed.

\subsection{Results and Discussion}

\subsubsection{Velocity Fields}

Velocity fields near the spark plug were obtained over 100 consecutive engine cycles. A partial sequence from a single cycle is shown in Figure 4-3. Image recording and start of injection were triggered at $45^{\circ} \mathrm{BTDC}$ and $43^{\circ} \mathrm{BTDC}$, respectively. Images 43a and 4-3b show two instantaneous velocity fields captured before the spray arrival. The upward motion of the piston and the tumble flow that is induced during the intake stroke through the intake valves govern the overall flow pattern. 

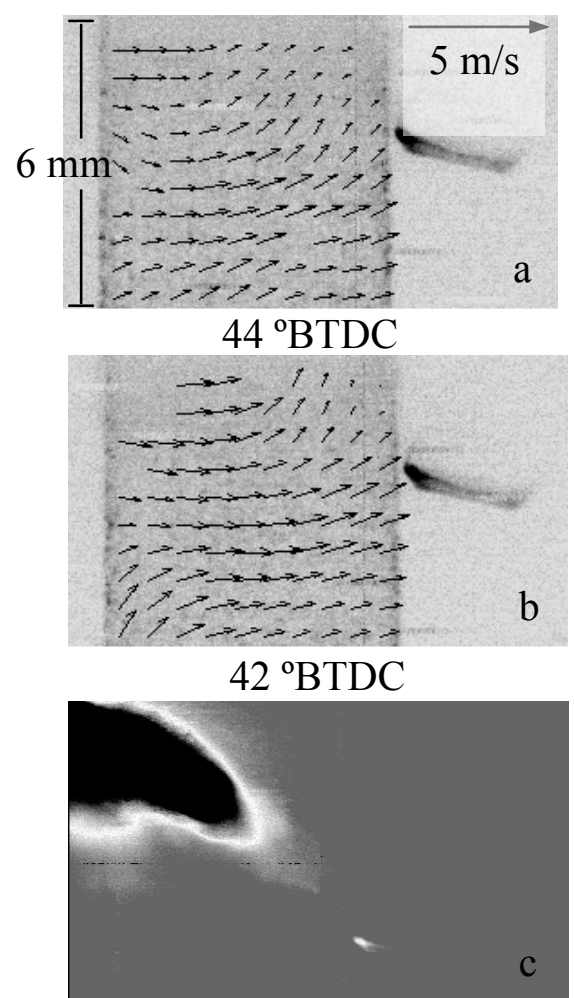

$40{ }^{\circ} \mathrm{BTDC}$

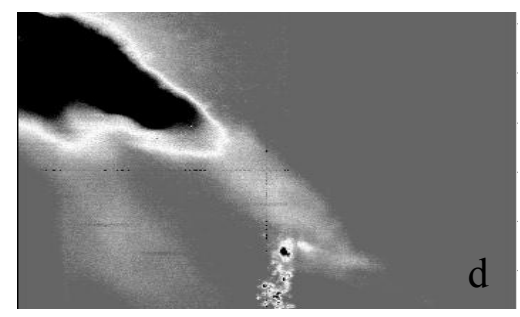

$38^{\circ} \mathrm{BTDC}$

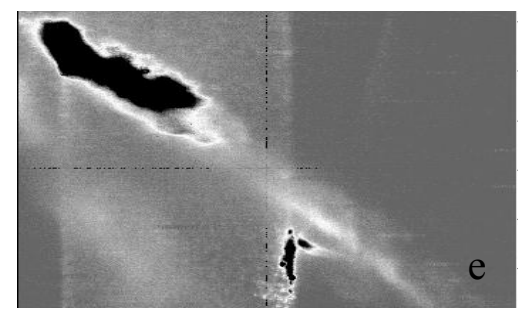

$36^{\circ} \mathrm{BTDC}$

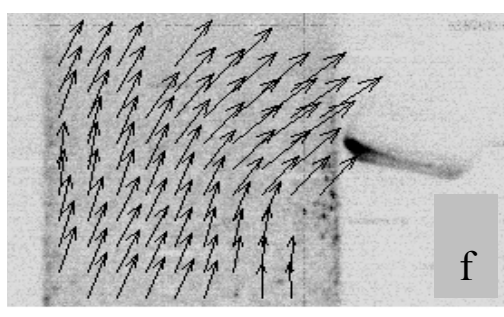

$34^{\circ} \mathrm{BTDC}$

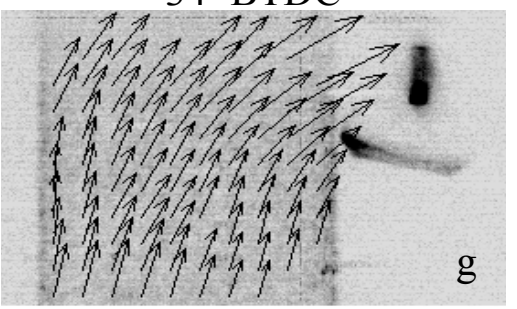

$33{ }^{\circ} \mathrm{BTDC}$

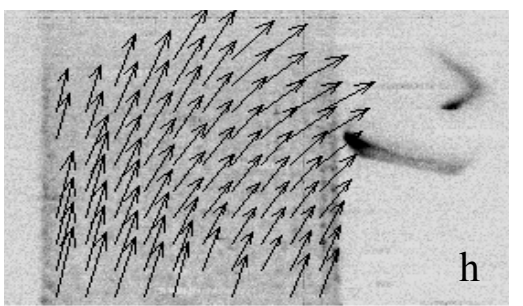

$32{ }^{\circ} \mathrm{BTDC}$

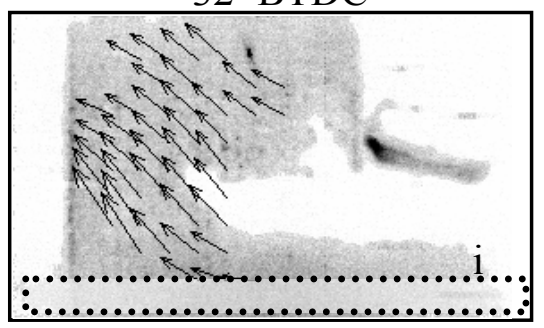

$26^{\circ} \mathrm{BTDC}$

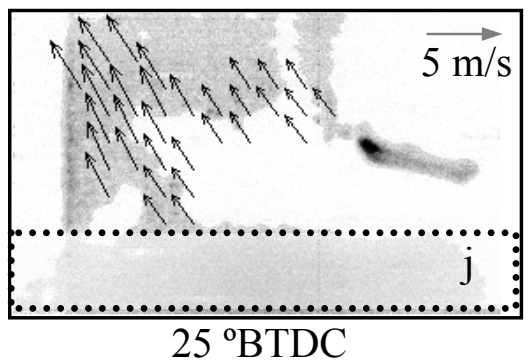

Figure 4-3 Sequence of instantaneous velocity fields from fuel injection to early flame propagation. Mie scattering signal from the spray prevents velocity field evaluation from $40{ }^{\circ} \mathrm{BTDC}$ to $35^{\circ} \mathrm{BTDC}$. The deformation of the plasma channel has been recorded. The dotted line indicates the location of the side piston window. Defocusing prevents imaging in this area. Notice the velocity scale change in (i) and (j). 
Starting at $40{ }^{\circ} \mathrm{BTDC}$, the strong Mie scattering signal from the spray prevented the evaluation of velocity data in the current field of view until the piston reached 35 ${ }^{\circ}$ BTDC. For illustration purposes, spray images are also displayed in the vector field sequence to show the effect that the spray exerts on the flow field structure (Figures 4-3c through 4-3e). Image $\mathrm{f}$, recorded after the end of injection, shows that in this engine, the flow surrounding the spark plug is strongly affected by the fuel spray. Post injection velocities in the vicinity of the spark plug substantially increase and the air motion is strongly directed towards the spark plug electrode. The flow pattern is consistent in every recorded engine cycle; it can be assumed to result from the combined effects of air entrainment by the fuel jet [39] and the strong tumble motion that persists through the late stages of compression (Figure 4-2). The spark discharge, triggered at $34{ }^{\circ} \mathrm{BTDC}$, is visible at $33^{\circ} \mathrm{BTDC}$ (Figure 4-3g) and the arc is deformed in the flow direction (Figure 4-3h). The propagating flame front is visible at $26^{\circ} \mathrm{BTDC}$ and $25^{\circ} \mathrm{BTDC}$ (Figure 4-3i and $\mathrm{j}$ ). Optical access is available through the $25 \mathrm{~mm}$-thick side piston windows, outlined in the last two images. The optical path however, increases considerably due to the window thickness, which coupled with the short depth of focus $(\sim 2 \mathrm{~mm})$ prevented focused imaging in this area. At the magnification and f-number required, the additional quartz window in the piston crown leads to defocusing and hence, blurred particle images [30]. In contrast to the PIV results presented in the previous chapter, detection of the strong soot luminosity has been suppressed by using $355 \mathrm{~nm}$ laser light and a short exposure time (i.e. $50 \mu \mathrm{s})$ for all frames, which makes the flame front easily identifiable. While the camera can be shuttered down to $2 \mu \mathrm{s}$, a $50 \mu \mathrm{s}$ exposure time was chosen to also image the emission from the spark discharge.

A quantitative display of flow velocity increase when the spray passes through the image area in the vicinity of the spark plug is shown in Figure 4-4. Square symbols show spatial velocity magnitude averages at each crank angle over 100 cycles. An increase by a factor of three in the average velocity magnitude and a noticeable increase in the RMS 
deviation of the average velocity (shown as error bars) near the spark plug after end of injection is apparent. The four spatially averaged, instantaneous velocity values per crank angle for the cycle that is shown in Figure 4-3 have also been displayed.

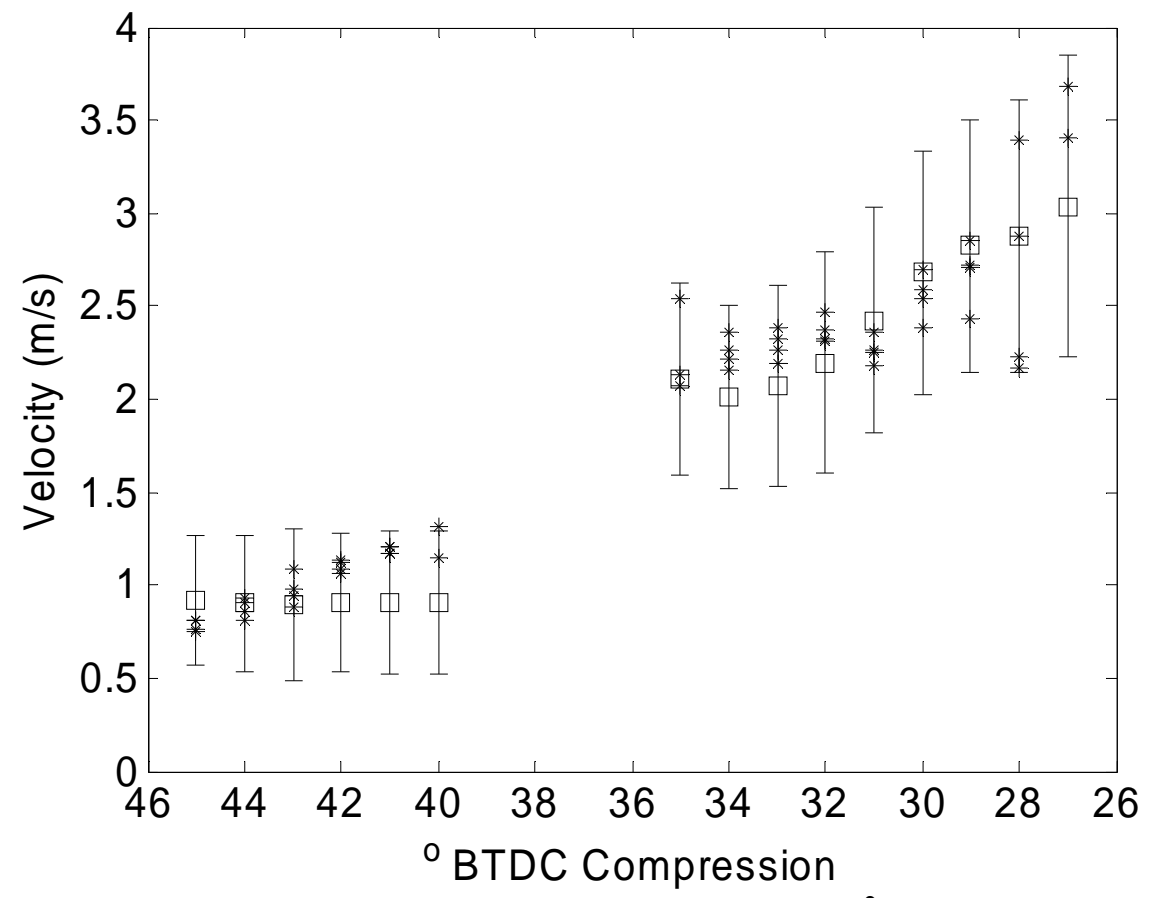

Figure 4-4 Spatially averaged velocity magnitude in a $5 \mathrm{~mm}^{2}$ area to the left of the spark plug. Legend: * four instantaneous values per crank angle for one of 100 cycles, $\square$ average over all cycles.

\subsection{2 $\quad$ Strain Rates}

The in-plane shear strain rate $\left(e_{12}\right)$ and two normal strain rate components $\left(e_{11}\right.$ and $e_{22}$ ) corresponding to each instantaneous flow field were calculated. The absolute value of the sum of $e_{11}$ and $e_{22}$ for each velocity field was also computed. Crank angle averaged results of are shown in Figure 4-5, along with the instantaneous values corresponding to the cycle displayed in Figure 4-3. 


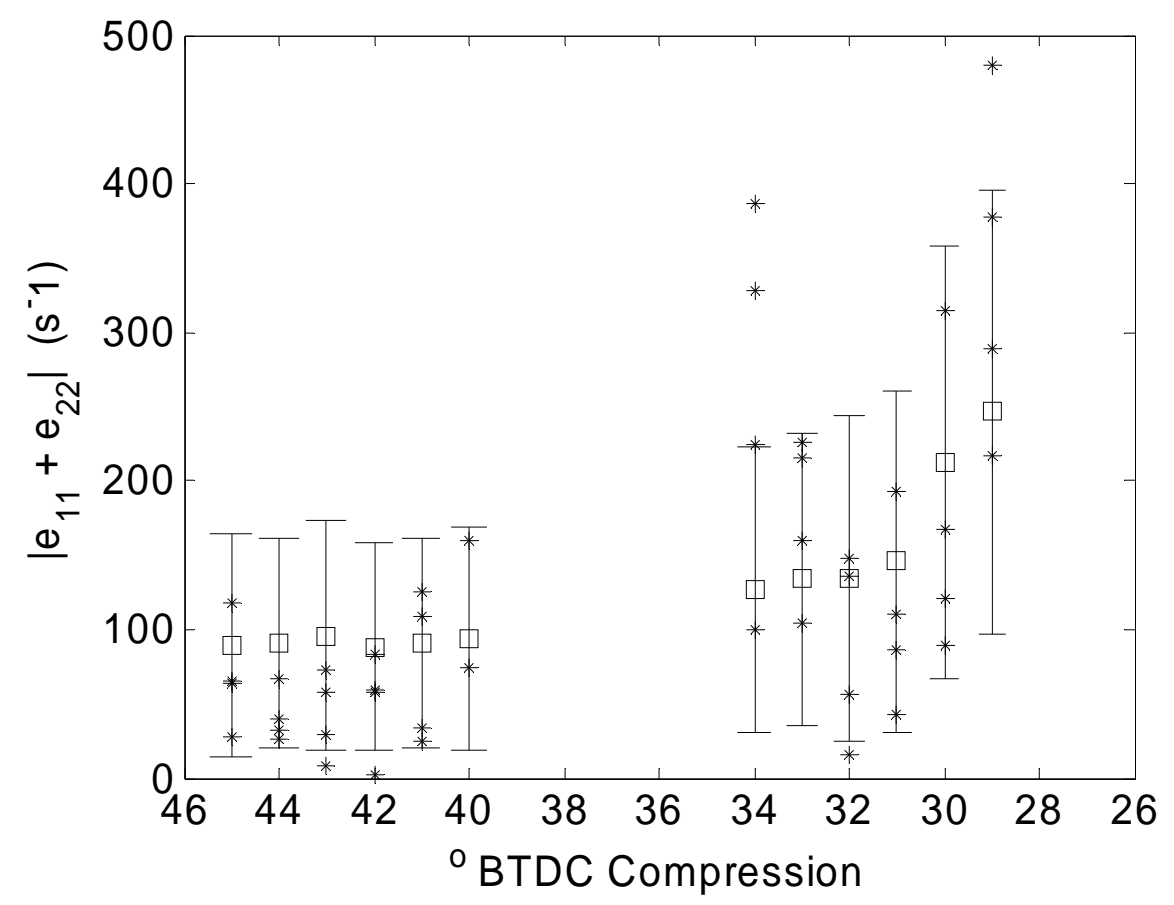

Figure 4-5 Spatially averaged sum of the normal strain rates. Legend: * four instantaneous values per crank angle for one of 100 cycles, $\square$ averaged over all cycles.

Internal combustion engine flows are largely three-dimensional. When largescale motion is induced either on the plane perpendicular to the cylinder axis (swirl) or parallel to it (tumble) the in-plane velocity components might become dominant. If under these conditions the flow can be considered quasi two-dimensional, velocity data recorded using planar measurement techniques (such as planar PIV) might not introduce significant uncertainty in the measured velocities. As shown in the previous chapter, the tumble flow induced during intake persists through the late stages of the compression (Figure 4-2) The current time-resolved data can provide insight regarding the quasi twodimensionality of the flow over the crank angle range under study. Neglecting spatial density variations, the continuity equation can be written as shown in (4-2).

$-\frac{1}{\rho} \frac{\partial \rho}{\partial t}=\frac{\partial u}{\partial x}+\frac{\partial v}{\partial y}+\frac{\partial w}{\partial z}$ 
The temporal density variation $\left(\rho^{-1} \partial \rho / \partial t\right)$ due to the upward piston motion can be approximated under the ideal-gas assumption, assuming polytropic compression $(\mathrm{k} \approx 1.25)$ and negligible blowby. The cylinder volume at each crank angle is available based on engine geometry and can be substituted into Equation (4-4). This yields an estimate of $100 \mathrm{~s}^{-1}$ for $\rho^{-1} \partial \rho / \partial \mathrm{t}$ at $45^{\circ} \mathrm{BTDC}$. Values on the same order of magnitude have been calculated in a similar engine [19].

$$
\begin{aligned}
& \frac{1}{\rho} \frac{\partial \rho}{\partial t} \sim \frac{1}{\rho} \frac{\Delta \rho}{\Delta t} \sim \frac{1}{\Delta t} \frac{\rho_{2}-\rho_{1}}{\rho} \\
& \frac{1}{\rho} \frac{\partial \rho}{\partial t} \sim \frac{1}{\Delta t} \frac{\left(\frac{V_{1}}{V_{2}}\right)^{k}-\left(\frac{V_{1}}{V_{2}}\right)^{k-1}}{\left(\frac{V_{1}}{V_{2}}\right)^{k-1}}
\end{aligned}
$$

Equations (4-1) and (4-2) can be combined to relate the normal strain rates to the temporal rate of change of the air density inside the cylinder. This yields the expression shown in Equation (4-5). If the derivative of the out-of-plane velocity component equals zero, then the $\rho^{-1} \partial \rho / \partial t$ term should equal the absolute value of the sum of the normal strain rate components. Figure 4-5 shows that the averaged experimental values of $\left|e_{11}+e_{22}\right|$ agree with this estimate prior to fuel injection, but not after the flame begins to develop. The effect of heat release, as evident from the increase in the $\left|e_{11}+e_{22}\right|$, can be seen after $32{ }^{\circ} \mathrm{BTDC}$.

$-\frac{1}{\rho} \frac{\partial \rho}{\partial t}=e_{11}+e_{22}+\frac{\partial w}{\partial z}$

In approximately $50 \%$ of the engine cycles involved in this study, an increase in the shear strain rate near the spark plug was noted. Figure 4-6 shows the progression of the shear strain rate fields corresponding to the velocity field sequence shown in Figure 4-3. Steep gradients can be seen across the field of view, and the values increase after fuel injection. 


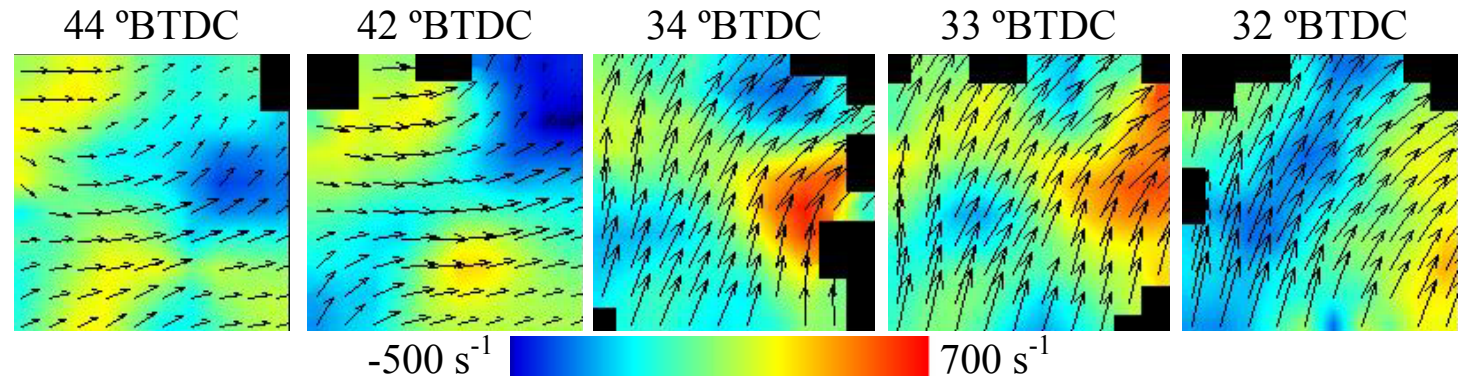

Figure 4-6 Sequence of instantaneous shear strain rate fields for the velocity fields shown in Figure 4-3. No values were computed in the black areas shown.

\subsection{Conclusions}

This chapter described the first application of sub-crank angle resolution UV PIV in a fired, spray-guided, stratified SIDI engine. The new features of this technique are (1) the use of $355 \mathrm{~nm}$ laser light and (2) acquisition of velocity data at the rate of four velocity fields per crank angle at 600 RPM engine speed.

These two features were achieved by using a single, frequency tripled Nd-YAG laser and a CMOS camera both operated at $16 \mathrm{kHz}$. Part of the motivation for using 355 $\mathrm{nm}$ laser light for PIV measurements, as summarized at the end of Chapter 3, was to suppress the detection of undesired combustion product luminosity and to capture the luminosity from the spark discharge. Results from the high-speed UV PIV experiment demonstrated that both goals can be achieved.

Velocity fields have been obtained over 100 consecutive cycles, from $45^{\circ}$ BTDC to $25^{\circ} \mathrm{BTDC}$. This crank angle range gives access to velocity data during a portion of the cycle vital to SIDI engine operation: from fuel injection through flame initiation. Data obtained over this crank angle range in our SIDI engine was used to assess the quasi twodimensionality of the flow. Experimental results also showed that post fuel injection velocity magnitudes near the spark plug increase by a factor of three and revealed 
pronounced displacement of the plasma channel in the flow direction. An increase in the shear strain rate was observed in approximately $50 \%$ of the cycles.

Due to reductions in laser energy output and camera image format at frequencies higher than $16 \mathrm{kHz}$, a single laser is not sufficient to conduct high-speed UV PIV measurements in the SIDI engine at 2000 RPM. Adding a second high-speed laser would provide flexibility to adjust the delay between laser pulses to match the flow speed at 2000 RPM. The lasers could be operated at a lower frequency for optimum performance and energy output. In addition, the field of view could be increased while maintaining the same spatial resolution. The anticipated drawbacks from adding a second laser include (1) higher complexity in synchronizing the two-laser, one-camera system with the optical engine and (2) more complex and time-consuming aligning procedure due to the addition of beam-combining optics and the short depth of field associated with the current setup.

The third main motivation for the development of the high-speed PIV technique using $355 \mathrm{~nm}$ light was the possibility to combine it with high-speed fluorescence of biacetyl for simultaneous imaging of the flow and equivalence ratio distribution. Due to the non-trivial drawbacks associated with adding a second laser, it was first decided to test the interaction of the chemicals: silicon oil and biacetyl used as tracers for PIV and PLIF experiments, respectively. This experimental investigation will be described in the next chapter. 


\section{CHAPTER 5}

\section{SIMULTANEOUS HIGH-SPEED UV PIV AND PLIF IN A COLD AIR JET TO}

\section{STUDY TRACER INTERACTION}

Recent investigations have demonstrated the feasibility of measuring gas-phase equivalence ratio distributions in engines at sustained frame rates of $12 \mathrm{kHz}$ using biacetyl as the fuel tracer [37]. In the previous chapter it was also shown that PIV can be applied to measure velocity fields in engines at frame rates of $16 \mathrm{kHz}$. These experiments, conducted separately, but under similar engine operating conditions, have provided data spanning the time period of injection, ignition and early flame propagation. Before combining high-speed UV PIV and biacetyl-PLIF for SIDI engine studies, it was important to verify that the tracers (i.e. silicon oil and biacetyl) could be used simultaneously. This chapter describes a study that combines high-speed PLIF and UV PIV using a single-laser, dual camera setup to obtain two-dimensional, simultaneous velocity-scalar data in a cold air-jet. The study also includes an assessment of the following three objectives:

1. Evaluate whether physical processes (e.g. adsorption, diffusion) that would cause biacetyl (PLIF tracer) and silicon oil droplets (PIV seeding) to interact are negligible. This can be determined by evaluating the strength and degree of spatial intermittency of the PLIF signal intensity for the seeding density required in PIV.

1. Determine the level of signal cross-talk (if any) between PLIF and PIV images. 
2. Evaluate whether the signal-to-noise ratio (SNR) is sufficient to obtain reliable velocity and scalar information in measurements in optical engines or similar devices.

By using the jet-flow arrangement, setup and synchronization of the diagnostic equipment are greatly simplified. Engine run time is reduced, thereby minimizing the wear of engine components. In addition, the selection of a straightforward and well characterized flow allows the isolation of tracer interaction in the absence of extraneous variables.

\subsection{Experimental Equipment and Setup}

The experimental is setup, schematically shown in Figure 5-1 includes a single, frequency-tripled diode-pumped Nd:YAG laser (Quantronix Hawk) operated at $12 \mathrm{kHz}$ and two high-speed CMOS cameras (Vision Research Phantom 7.1). At $12 \mathrm{kHz}$, the laser provides consecutive pulses of $100 \mathrm{~ns}$ duration and approximately $0.5 \mathrm{~mJ}$ of energy.

The laser pulse frequency was selected to meet the requirements for crank angle resolution at $2000 \mathrm{RPM}$ engine speed. Since the laser energy varies with pulse frequency, selecting $12 \mathrm{kHz}$ allows one to evaluate whether the intensity levels of the scatter and fluorescence signals are enough at this temporal resolution. Since the camera full frame resolution ( $800 \times 600$ pixels) is achieved at $4.8 \mathrm{kHz}$, the recording area was reduced to $416 \times 416$ pixels at $12 \mathrm{kHz}$, corresponding to a $20 \times 20 \mathrm{~mm}^{2}$ in the object plane. Approximately 32,500 consecutive frames could be recorded.

The $355 \mathrm{~nm}$ laser beam was reflected using a HR 355/HT $53245^{\circ}$ turning mirror. Using light-sheet forming optics (Rodenstock Modular Focus), the beam was focused and expanded into a $1 \mathrm{~mm}$ thick light sheet that illuminated the centerline of the jet. For PIV, air was mixed with silicon oil droplets (Dow Corning 510R) produced by a six-jet atomizer (TSI Model 9306). This is the same tracer and apparatus used in the PIV 
experiments described in Chapters 3 and 4. To introduce the biacetyl, line air was routed through a bubbling apparatus. As the air passed through liquid biacetyl a bypass valve was used to adjust its flow rate and in turn regulate the recorded biacetyl signal. Both the biacetyl-air and oil-air streams merged into a single line and mixed over a length of 20 $\mathrm{cm}$ before reaching a circular, $7 \mathrm{~mm}$ inner diameter stainless steel pipe oriented with its longitudinal axis along the $z$ direction (see Figure 5-1).

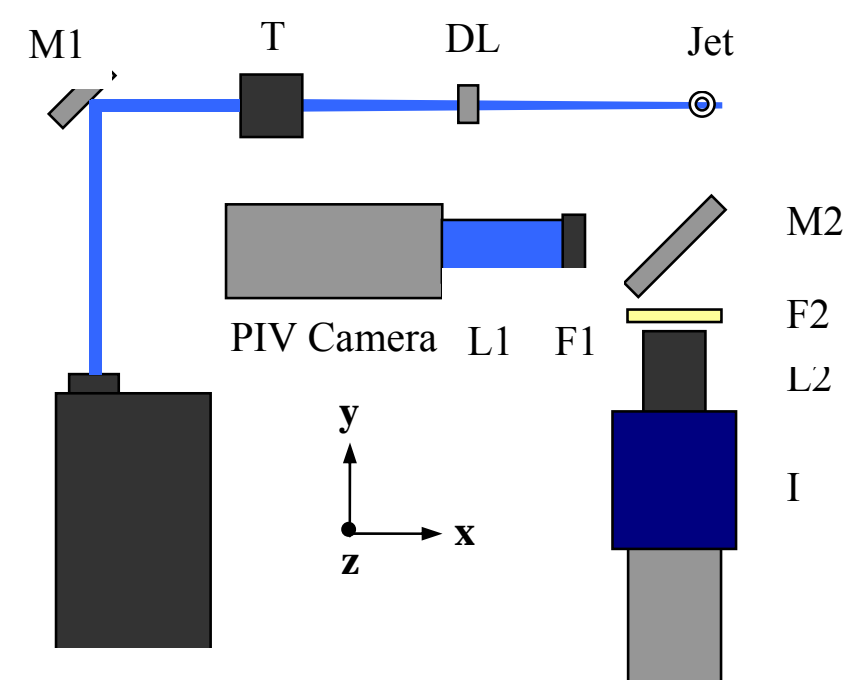

Nd:YAG laser

\section{PLIF Camera}

Figure 5-1 Setup for the velocity-scalar measurements in a cold jet using $355 \mathrm{~nm}$ excitation light. Legend: M1-HR355 HT 532 mirror, M2-HR 355 mirror, T-focusing optics, DL-diverging lens, L1-105 mm UV lens, L2-105 mm Nikon Micro-Nikkor lens, F1-355 nm interference filter, F2-GG 420 filter, I-image intensifier.

The oil-air and biacetyl-air flow rates were adjusted to provide enough signal for the PLIF measurement and the required seeding density for PIV. As for the previous PIV setups, the seeding density was optimized by adjusting the atomizer pressure until 10 to 15 particle images were contained in $32 \times 32$ pixel areas. It was also important to ensure that the exit flow speed could be resolved with the $83.3 \mu$ s (i.e. $1 / 12 \mathrm{kHz}$ ) laser pulse delay. This was accomplished by adjusting the flow rate until the maximum particle shift 
between laser pulses did not exceed one quarter of the interrogation window size $[26,29,40]$.

A $101 \mathrm{~mm}$ HR 355/45 degree mirror was placed in front of the CMOS cameras. The elastically scattered light from the $1 \mu \mathrm{m}$ diameter oil droplets used for the PIV measurements was reflected via this mirror and focused by a $105 \mathrm{~mm} \mathrm{f} / 4.5 \mathrm{UV}$ lens (Coastal Optics SLR) onto the first (PIV) camera. A $355 \mathrm{~nm}$ interference filter was placed in front of the PIV camera to minimize the level of background signal recorded. Biacetyl fluorescence was collected with a $105 \mathrm{~mm}$ f/1.4 lens (Nikon Micro-Nikkor) and recorded by a second camera that was coupled with a high-speed image intensifier (LaVision HS-IRO). An $80 \mathrm{~mm}$ x $80 \mathrm{~mm} \mathrm{GG} 420$ long-pass filter was placed in front of the fluorescence imaging setup to suppress both scattered laser light and a portion of the spark discharge signal, which can damage the image intensifier. The biacetyl fluorescence band extends between 420 and $550 \mathrm{~nm}$. Consequently, the GG 420 filter does not significantly reduce PLIF signal levels.

A pulse generator (Stanford Research Systems DG 535) was used to trigger the cameras and the laser at $12 \mathrm{kHz}$ (Figure 5-2). Previously, it was shown that setting the camera exposure time to $50 \mu \mathrm{s}$ suppresses the detection of combustion product luminosity while capturing the spark signal. The exposure time for the combined PIVPLIF experiments was set to $40 \mu \mathrm{s}$. To record the biacetyl fluorescence, the image intensifier was triggered with the PLIF camera during the $40 \mu$ s exposure time. The delay between the intensifier trigger pulse and gate is negligible. As a result, to ensure that the laser pulse arrived during the short intensifier gate time $(1.35 \mu \mathrm{s})$ the delay between the clock trigger and laser pulse was adjusted while observing the strength of the PLIF signal. The optimum setting for this delay was $14 \mu \mathrm{s}$. The PIV camera trigger was synchronized with the PLIF trigger to ensure that the scatter and fluorescence signals were recorded simultaneously. 


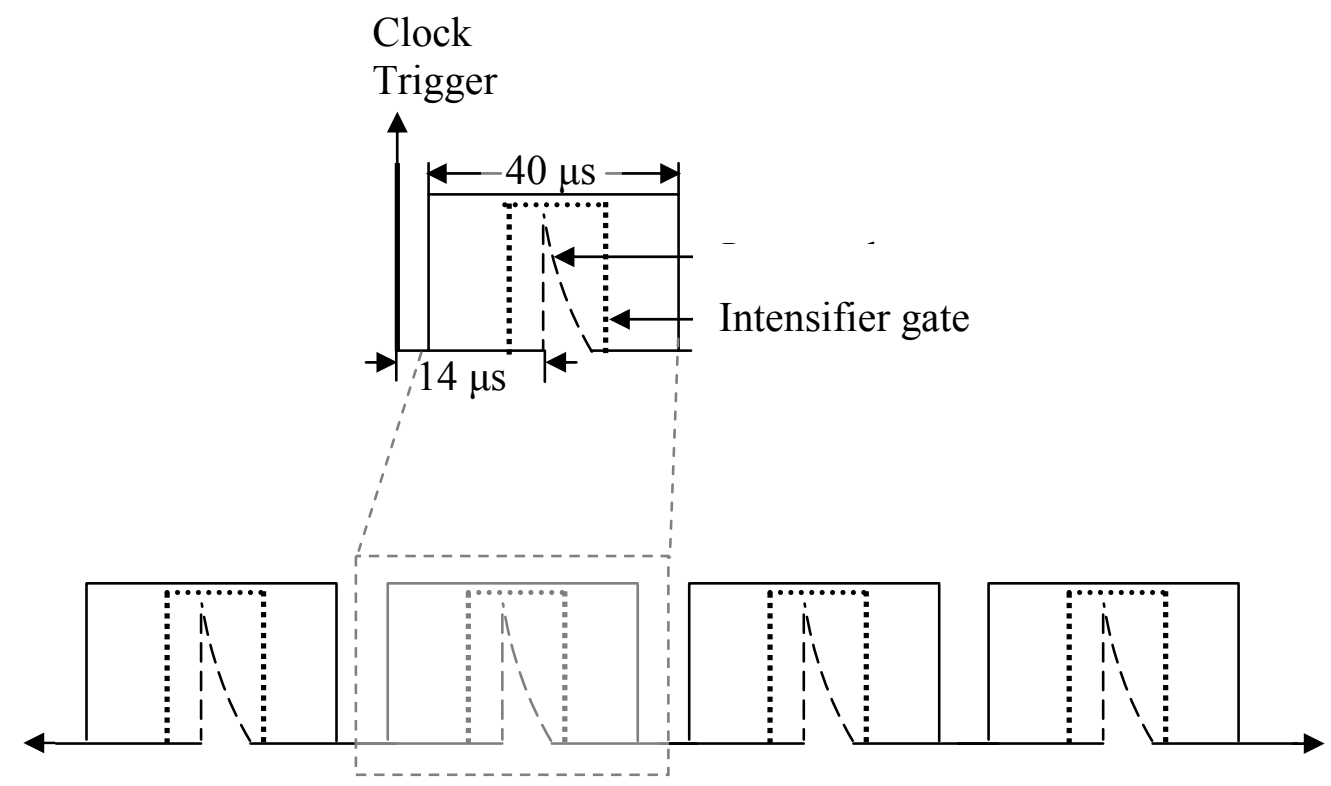

Figure 5-2 Triggering scheme for the simultaneous application of high-speed PLIF and PIV to a jet flow.

\subsection{Data Processing}

Consecutive, instantaneous images were cross-correlated using a commercial software package (LaVision $\mathrm{DaVis}$ 7.1). A mask was applied to the raw images to exclude unseeded areas in the periphery of the jet during processing. Velocity vectors were computed inside the mask using FFT-based cross-correlation algorithm. The interrogation process was performed in three steps using decreasing window sizes from $128 \times 128$ pixels to $32 \times 32$ pixels with $50 \%$ overlap. The in-plane spatial resolution based on the $32 \times 32$ pixel interrogation spot was $1.6 \mathrm{~mm}$. A standard deviation operator was applied to replace spurious vectors with the mean of the four adjacent vectors. As a final step during post processing, a $3 \times 3$ top-hat filter was applied to the vector fields to minimize error propagation when computing velocity gradients. These gradients were calculated by taking first-order differences of the smoothed velocity field at each point. 


\subsection{Results and Discussion}

For demonstration of the simultaneous concentration and velocity imaging technique, data at a single axial location are discussed below. The jet Reynolds number was estimated based on velocity data extracted from PIV recordings $2 \mathrm{~mm}$ above the nozzle exit. Using the ensemble average velocity at this location $(\sim 4 \mathrm{~m} / \mathrm{s})$, the pipe inner

diameter $(\sim 7 \mathrm{~mm})$, and the kinematic viscosity of air $\left(\sim 1.4 \times 10^{-5} \mathrm{~m}^{2} / \mathrm{s}\right)$, the Reynolds number $(\mathrm{Re})$ is calculated to be 2000 .

The left column in Figure 5-3 shows a sequence of velocity-scalar images that were recorded simultaneously $27 \mathrm{~mm}$ downstream of the nozzle. The time separation between successive images is $83.3 \mu$ s, matched to the requirements for best PIV evaluation. However, to visually highlight the changes in the flow, images are skipped in the display of Figure 5-3. Note the timestamp on the images as well as the value indicating the frame number in the sequence of the original recording. In all images, the absolute velocity field has been superimposed onto the scalar field. The middle column shows the same sequence, but in this case, the velocity has been displayed relative to a fixed seven pixel displacement to reveal large-scale vortices arising from flow instabilities. The corresponding in-plane shear strain rate is shown on the right column of Figure 5-3. As expected, the largest strain values are localized along the jet shear layer, where the velocity gradients are largest. 

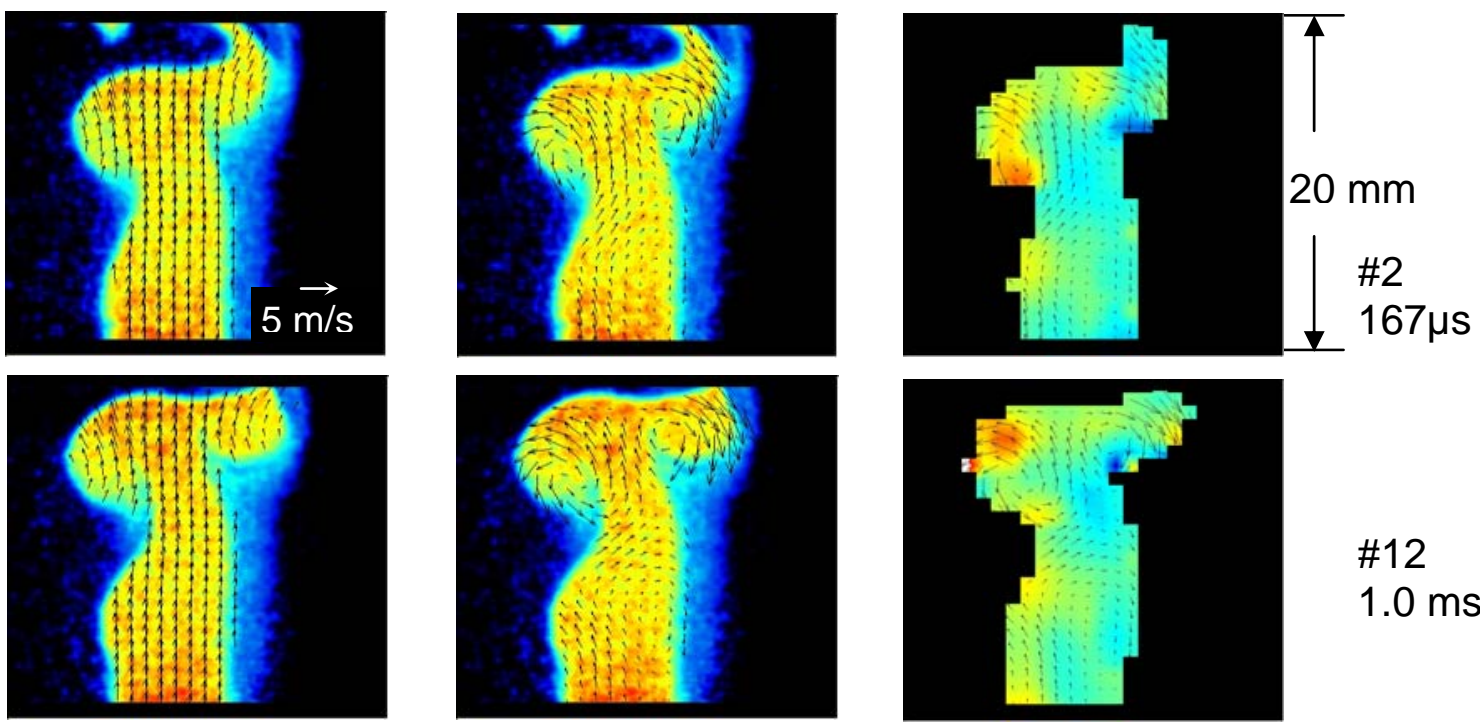

\#12

$1.0 \mathrm{~ms}$
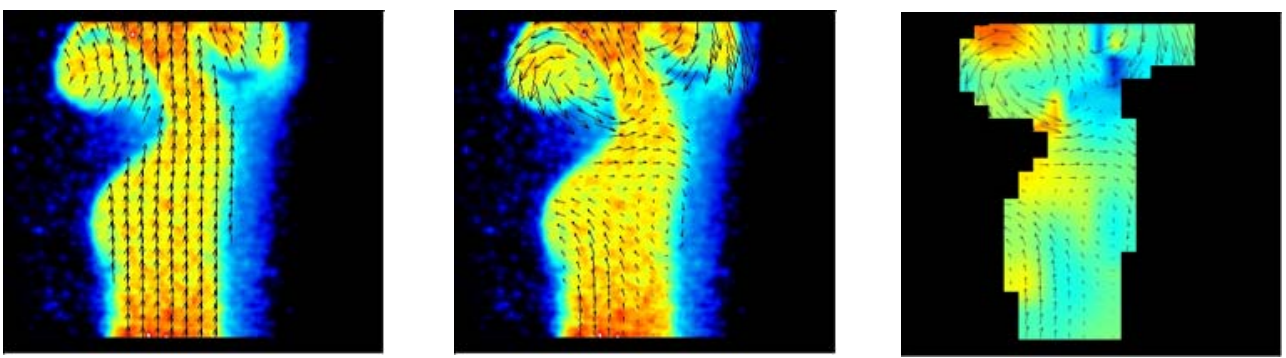

\#22

$1.8 \mathrm{~ms}$
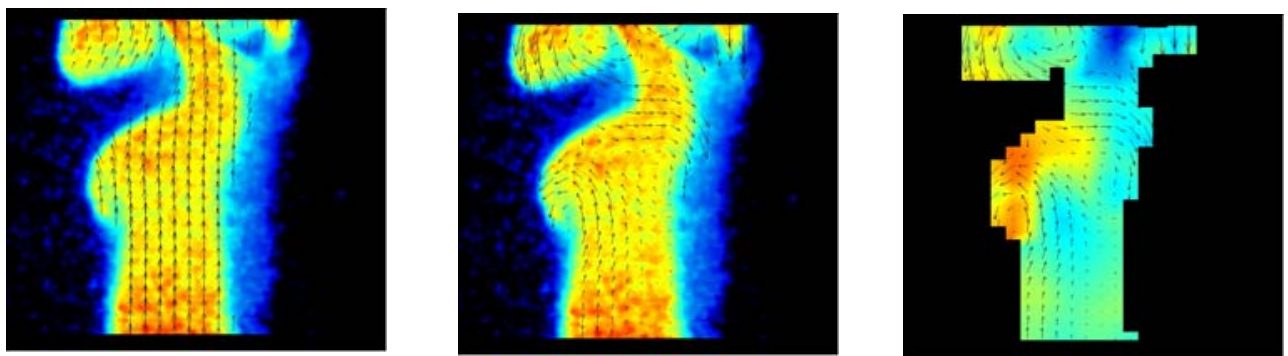

\#33

$2.7 \mathrm{~ms}$

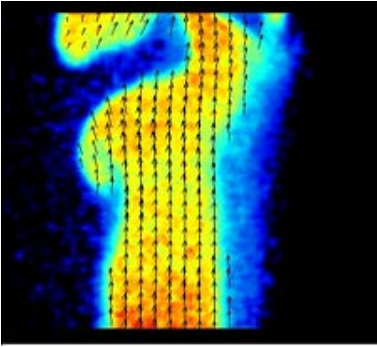

0

Absolute velocities and concentration

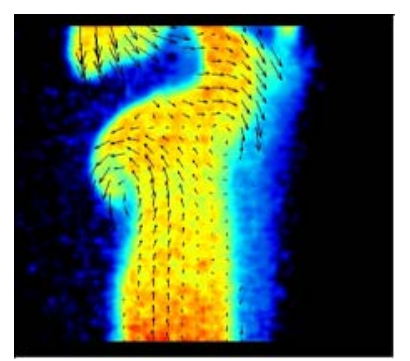

2

Relative velocities and concentration

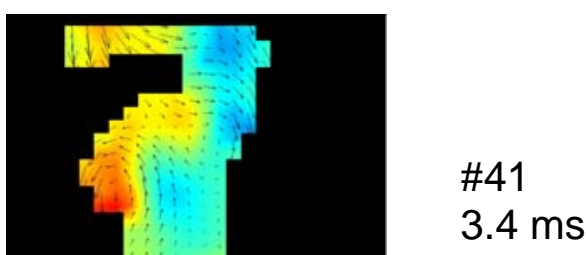

\section{$-700 s^{-1}$}

Relative velocities and strain rate

Figure 5-3 Velocity-scalar fields obtained with high-speed PIV and biacetyl PLIF 


\subsection{Evaluation of Tracer Interaction}

Based on the liquid oil to gas volumetric ratio, physical processes such as adsorption and diffusion of biacetyl into the silicon oil are not expected to contribute significantly to PLIF signal degradation. The interrogation volume, containing a wellmixed biacetyl-air blend and the oil droplets used as the PIV tracer, can be estimated as $2.56 \mathrm{~mm}^{3}$ when using the interrogation window size of $32 \times 32$ pixels $(1.6 \mathrm{~mm} \times 1.6 \mathrm{~mm})$ and light sheet thickness $(1.0 \mathrm{~mm})$ for the PIV measurement. This volume encloses 15 (approximately spherical) oil droplets $1 \mu \mathrm{m}$ in diameter. The oil volume is then $\sim 8 \mu \mathrm{m}^{3}$, yielding an oil-to-biacetyl-air volume ratio of $\sim 3 \mathrm{ppb}$. This value suggests that the tracer interaction should be negligible. Influence of the PIV seeding on the biacetyl would be noticed as more intense points scattered about the field of view in the PLIF images. No qualitative difference in the PLIF images was observed with or without the presence of PIV oil droplets.

To evaluate whether there was signal cross-talk, the biacetyl-air stream was temporarily blocked, allowing only the oil-seeded air to exit the pipe. In the absence of biacetyl, the 12-bit PLIF camera recorded a maximum signal of 175 counts (i.e. less than $15 \%$ of the maximum PLIF signal of 1200 counts). It is thought that the recorded signal results from a small fraction of $532 \mathrm{~nm}$ light being scattered by the oil droplets. Despite the HT $532 \mathrm{~nm}$ coating of the turning mirror (M1 in Figure 5-1) a small amount of the residual green light in the laser output gets reflected. To test this hypothesis, a polarizing beam-splitting cube was placed in the laser beam path after M1 to separate the $355 \mathrm{~nm}$ from the $532 \mathrm{~nm}$ components, which are vertically and horizontally polarized, respectively. The separated beams clearly showed the presence of the $532 \mathrm{~nm}$ component. Future improvements of the setup therefore could include a Pellin-Broca prism to replace M1. While this can be effectively used to eliminate the unwanted 532 $\mathrm{nm}$ light in the light sheet, it is worth noting for the images presented here that the Mie 
scattering signal recorded by the PLIF camera is on the order of the noise level of PLIF measurements.

A measure of the signal-to-noise (SNR) ratio for the PIV measurements is provided in Figure 5-4. The abscissa shows the average quality (Q) factor, which represents the ratio of signal-to-noise peaks in the correlation map. The fraction of vectors retained after post processing is quantified in the y-axis.

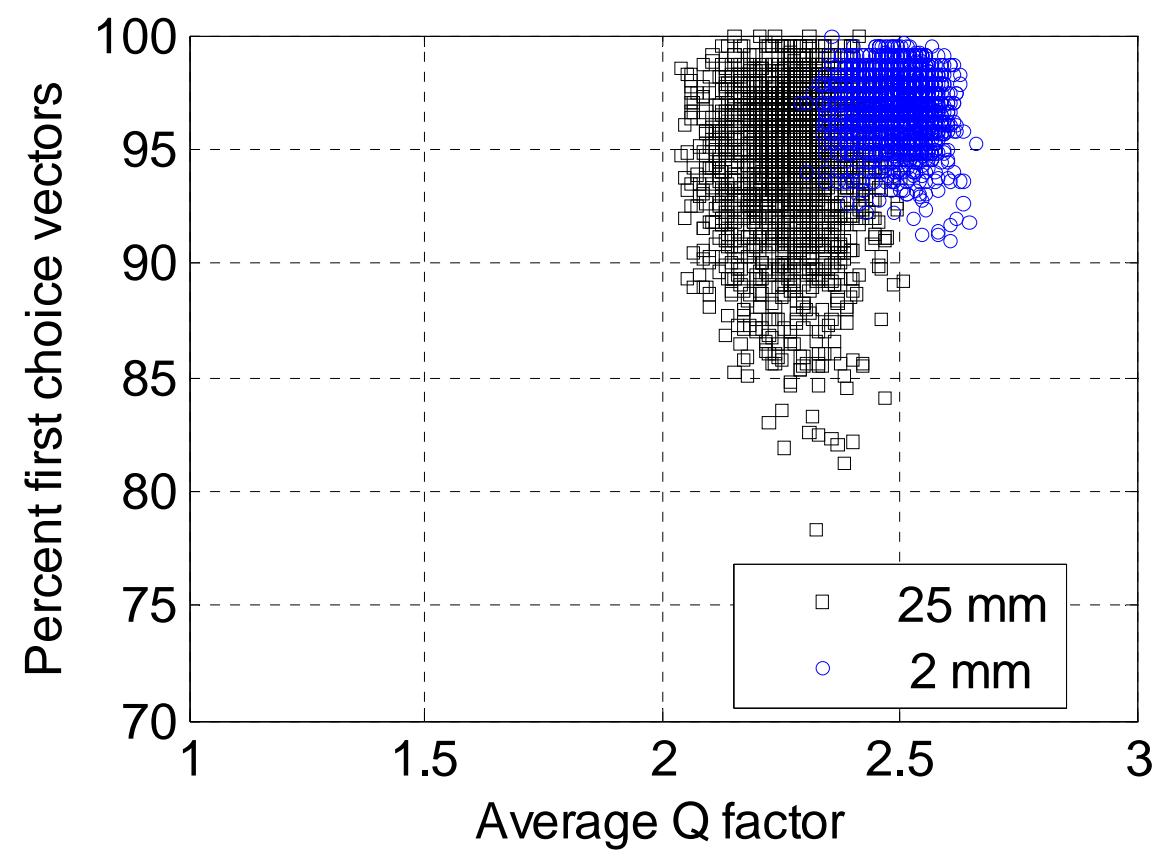

Figure 5-4 Average quality (Q) factor and percentage of vectors retained after postprocessing, corresponding to the velocity fields obtained with the single-laser, singlecamera, high-speed UV PIV technique. The Q factor, which represents the ratio of the signal-to-noise peaks in the correlation map, demonstrates the high quality of the PIV measurements. A decrease in $\mathrm{Q}$ is noted for areas with rapid changes in spatial extent of the jet. This is due to the adaptive masking scheme that was employed, leading to small areas where no overlapping seeded areas are found.

The data corresponds to 2000 vectors fields calculated at two axial locations. The data for location 1, shown with circles, belong to the pipe exit positioned $2 \mathrm{~mm}$ below the FOV. The data for location 2, shown with squares, correspond to the pipe exit positioned $27 \mathrm{~mm}$ below the FOV. In $90 \%$ of the images, the retained vectors after post processing exceed $95 \%$ and $90 \%$ for locations 1 and 2, respectively. The discrepancy in percentage 
of vectors retained between the two locations can be attributed to the continuous roll-up and shedding of vortices at location 2. This phenomenon alters the effective (masked) area where vectors are computed between consecutive images and thus results in weaker correlations. The average $\mathrm{Q}$ factor lies above two in all cases, indicating that strong correlations were found.

The SNR of the PLIF experiment was calculated as the ratio of the spatially averaged signal strength in the core of the jet to the average outside of the jet area. Instantaneous SNR values are on the order of 9:1. In contrast, SNR corresponding to biacetyl PLIF experiments conducted in the optical engine have generally been on the order of 5:1 [37]. Potential differences in the signal-to-noise ratio between PIV and PLIF experiments conducted in open-air and in the optical engine may be attributed to the presence of additional optical components in the excitation beam path (e.g. beamcombining optics) and also between the object and image planes (quartz engine cylinder).

\subsection{Conclusions}

This chapter demonstrated the first simultaneous application of particle image velocimetry and planar laser induced fluorescence of biacetyl using $355 \mathrm{~nm}$ as the excitation wavelength and at sustained frame rates of $12 \mathrm{kHz}$. Experimental results showed the feasibility of using silicon oil droplets and biacetyl as the PIV and PLIF tracers, respectively. No spatial intermittency was found in the PLIF signal for the oil concentration (seeding density) required for PIV. Adequate signal-to-noise ratio was achieved for both the PLIF and PIV measurements. Some signal cross-talk was detected by the PLIF camera due to Mie scatter of residual $532 \mathrm{~nm}$ light by the silicon oil droplets. While an improvement strategy for the current experimental setup has been provided, the intensity of the unwanted signal is on the noise level of the PLIF measurements. Both techniques have been demonstrated separately in the optical engine. Results from this 
tracer experiment suggest that high-speed UV-PIV and biacetyl PLIF can be successfully combined to obtain time-resolved instantaneous velocity and scalar fields in the SIDI engine. 


\section{CHAPTER 6}

\section{EFFECT OF PIV MEASUREMENT NOISE ON KINETIC ENERGY AND}

\section{DISSIPATION SPECTRA OF ENGINE FLOWS}

\subsection{Introduction}

Due to the complex nature of IC engine flows, assumptions and/or compromises must be made in many cases to investigate the flow either through computational or experimental investigations. In any case, it is important to know the limitations of each approach in order to draw meaningful interpretations from the results.

Kinetic energy and dissipation rate spectra provide valuable insight on the spectrum of turbulence and flow dynamics throughout the engine cycle, which is highly desirable to support the development of advanced combustion strategies and validate turbulence models. Spectral separation of in-cylinder flow structures has been achieved through spatial band-pass filtering [43] of the instantaneous, two-dimensional velocity fields. In [44], spectra of the spatially averaged turbulent kinetic energy and enstrophy were presented and compared with K-41 theory. Both spectra showed a roll-off near the $(1 \mathrm{~mm})$ resolution region. While it was suggested that data obtained with this resolution encompassed most of the inertial range, the authors emphasized the need for conducting PIV experiments with higher spatial resolution to determine the cause of the observed roll off. Such velocity data, obtained via PIV measurements at two spatial resolutions (i.e. 2 $\mathrm{mm}$ and $0.350 \mathrm{~mm}$ ) are presented and analyzed in this chapter to demonstrate the effect 
of measurement noise on the (two-dimensional) kinetic energy and dissipation rate spectra.

\subsection{Experimental Equipment and Setup}

The PIV data used in this analysis were measured at $60{ }^{\circ} \mathrm{BTDC}$ on plane perpendicular to the cylinder axis and $4 \mathrm{~mm}$ above the piston. The engine was motored in all cases. High and low swirl flows were induced by adjusting a swirl valve located in one of the intake ports. The intake temperature and pressure remained at $35{ }^{\circ} \mathrm{C}$ and 50 $\mathrm{kPa}$, respectively.

Two PIV setups were used to gather velocity data over different flow scale ranges. While the same equipment was used in both, the magnification, f-stop and light sheet thickness varied to achieve spatial resolutions of $2 \mathrm{~mm}$ and $350 \mu \mathrm{m}$, as shown in Table 6-1. Instantaneous images were acquired during the compression stroke at the fixed piston location.

Table 6-1 Experimental PIV Parameters

\begin{tabular}{|c|l|c|c|}
\hline $\begin{array}{c}\text { Engine Speed } \\
(\mathrm{RPM})\end{array}$ & Swirl Level & $\begin{array}{c}\text { Spatial Resolution } \\
(\mathrm{mm})\end{array}$ & Magnification \\
\hline 2000 & High/Low & 0.35 & 0.62 \\
\hline & High/Low & 2.00 & 0.11 \\
\hline
\end{tabular}

The experimental setup and beam alignment procedure are thoroughly described in [32] and briefly summarized here. A schematic representation is provided in Figure 6-1. 


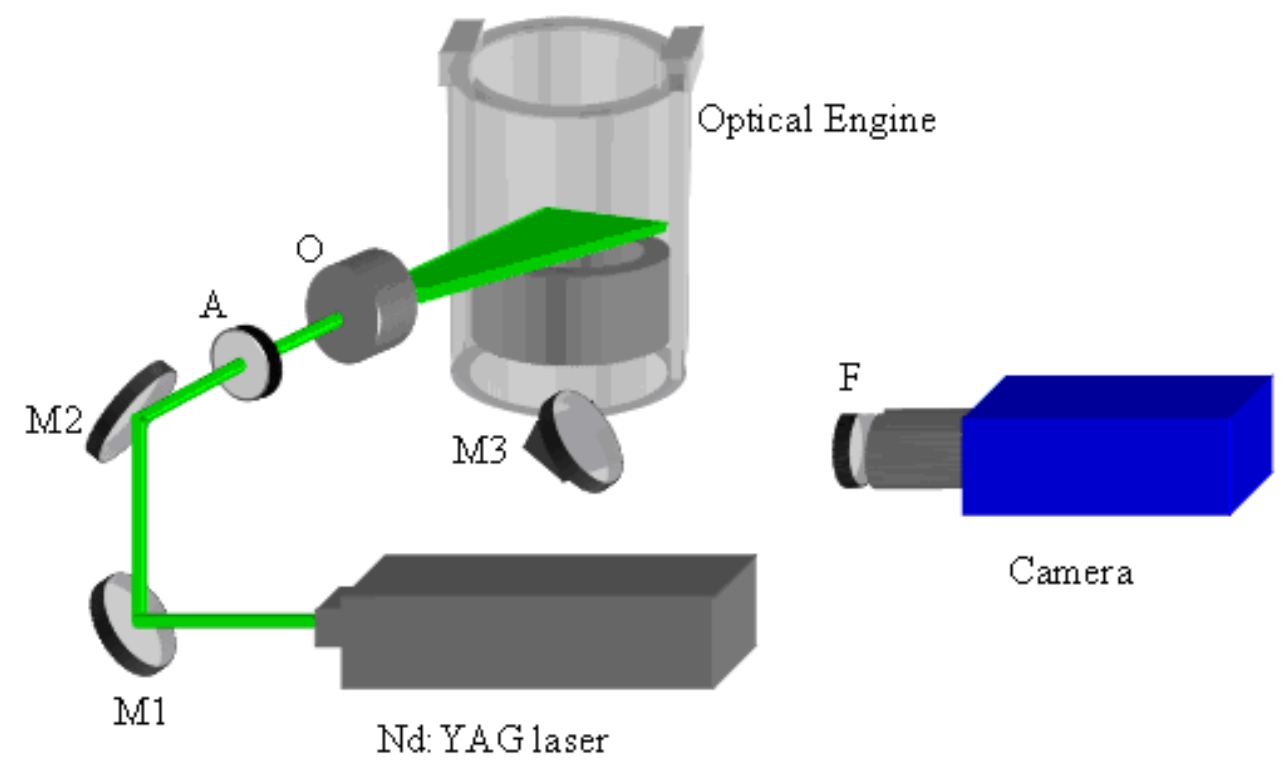

Figure 6-1 Experimental setup for the engine PIV measurements. F: narrow-band filter, O: lightsheet optics, M1,M2: dichroic mirrors, M3: turning mirror, A: aperture

A dual-resonator, frequency-doubled Nd:YAG laser (NewWave Minilase 15) produced $532 \mathrm{~nm}$ beam, which were focused as they passed through an objective with variable focal length containing two spherical lenses (Rodenstock Modular Focus). A $-10 \mathrm{~mm}$ cylindrical plano-concave lens was positioned at the exit of the objective to diverge the beams. For the low $(2 \mathrm{~mm})$ spatial resolution experiments, the Mie scattered light was imaged using a $105 \mathrm{~mm}$ Nikon MicroNikkor ED lens (f/5.6). The corresponding $80 \times 60 \mathrm{~mm}^{2}$ field of view (FOV) covered the entire imaging area accessible through the piston window with a diameter of $44 \mathrm{~mm}$. To increase the spatial resolution to $350 \mu \mathrm{m}$, a $200 \mathrm{~mm}$ Nikon ED AF MicroNikkon macro lens was used, equipped with $97 \mathrm{~mm}$ extension tubes. The magnification was fixed and translation stages were used to adjust the focus and to center the resulting $14 \times 11 \mathrm{~mm}^{2}$ FOV with respect to the imaging area used for the low resolution PIV experiments (Figure 6-2). In both setups, a $532 \mathrm{~nm}$ narrow-band filter was placed in front of the camera lens to isolate the $532 \mathrm{~nm}$ signal. 


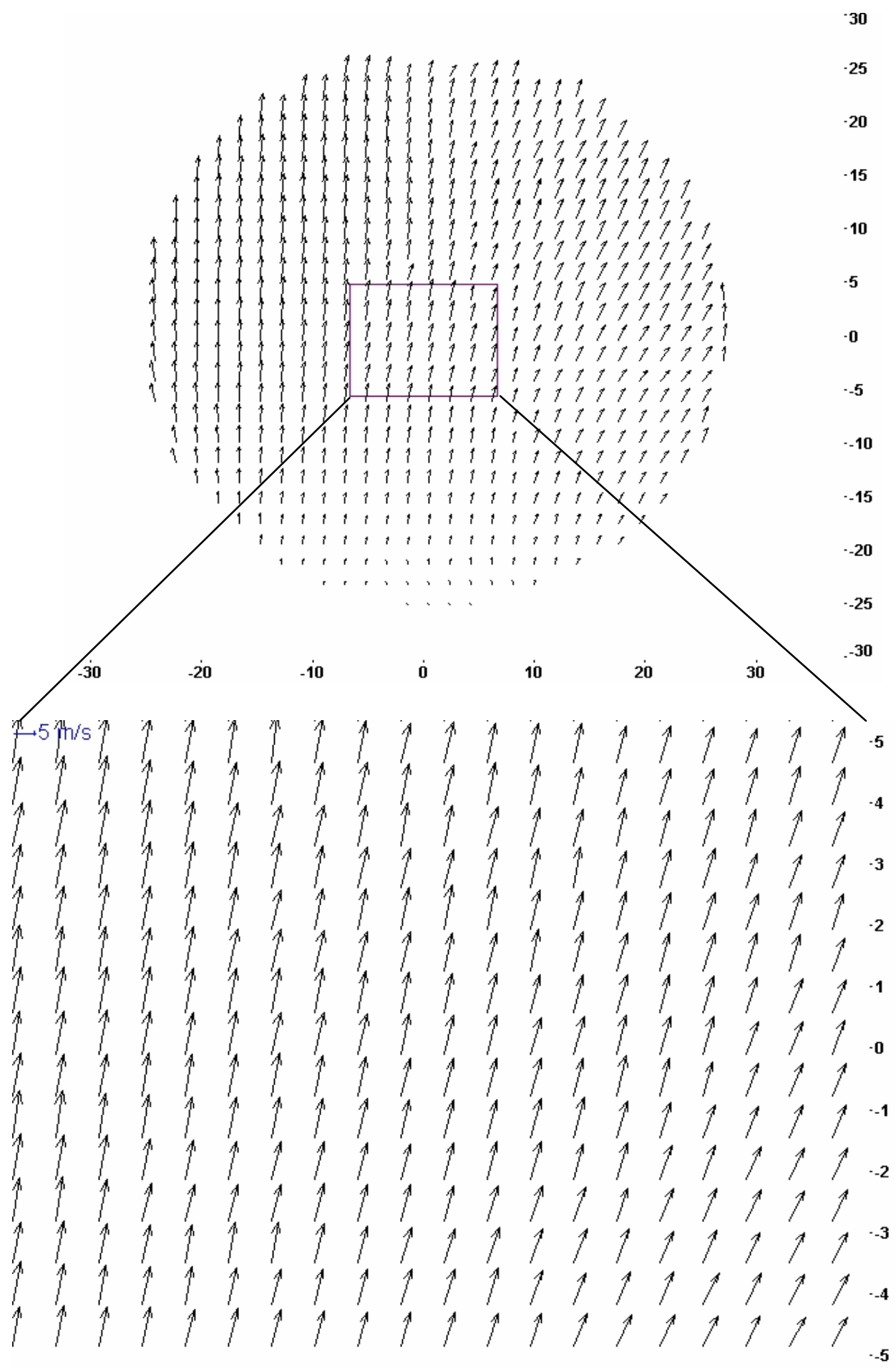

Figure 6-2 Location of the field of view used in the high resolution $(350 \mu \mathrm{m})$ PIV measurements (b) with respect to the field of view corresponding to the low resolution (2 $\mathrm{mm}$ ) experiments (a). Every second vector shown in (a) and every fourth vector is shown in (b). 


\subsection{Image Acquisition and Data Processing}

Instantaneous velocity fields were obtained from 300 double-frame images, acquired at the rate of two images every four engine cycles. A high pass filter was applied to eliminate low-frequency background fluctuations that arise from internal reflections in the cylinder. The filter size (10 pixels) was chosen to provide a nearly constant background without compromising the signal scattered by the tracer particles. The pre-processed images were then cross-correlated using a FFT-based, multiple-pass routine using a three-point Gaussian peak-fitting algorithm for sub-pixel estimation of particle location. A three-step cross-correlation method with decreasing window size was used, from $128 \times 128$ to $32 \times 32$ pixels with $50 \%$ overlap, yielding 300 instantaneous velocity fields with 16 pixel grid spacing. This corresponds to $0.95 \mathrm{~mm}$ for the low-resolution measurements and $0.17 \mathrm{~mm}$ for the high-resolution measurements. The quality of the resulting vector fields was validated by (1) the percentage of first choice vectors, (>95\% in $99 \%$ of the images) and (2) the ratio of signal to noise peaks in the correlation map $(\mathrm{Q}$-factor $=2)$. As the final optional step, a $3 \times 3$ top-hat smoothing filter was applied to the velocity fields. This filter computes the velocity at a point $\left(\mathrm{x}_{0}, \mathrm{y}_{\mathrm{o}}\right)$ as the average over the $3 \times 3$ point matrix centered at $\left(\mathrm{x}_{0}, \mathrm{y}_{\mathrm{o}}\right)$, applying equal weight to all the points. The effect of smoothing the velocity fields on the two-dimensional kinetic energy and dissipation rate spectra will be discussed with the results.

\subsubsection{Kinetic Energy and Dissipation Rate Spectra Calculations}

The flow structure at various spatial scales was isolated by band-pass filtering the instantaneous velocity fields using a Gaussian filter [43]. By using this filter, the velocity at a point $\left(\mathrm{x}_{0}, \mathrm{y}_{\mathrm{o}}\right)$ in the two dimensional flow map is computed as the Gaussian weighted average of the velocity at all points under the normal curve. The Gaussian weight for each location $\left(\mathrm{x}_{\mathrm{i}}, \mathrm{y}_{\mathrm{i}}\right)$ and a filter width $f$ at $1 / \mathrm{e}^{2}$ of the maximum is given as: 


$$
G\left(x_{i}, y_{i}\right)=\left\{\frac{1}{2 \pi f^{2}} \sqrt{\frac{2}{\pi^{3}}} \exp \left(\frac{-8}{f^{2}}\left[\left(x_{i}-x_{o}\right)^{2}+\left(y_{i}-y_{o}\right)^{2}\right]\right)\right\}
$$

The result of the Gaussian filtering process is a low-pass filtered velocity field (i.e. spatial frequencies below the filter size are smoothed out). By calculating the difference of velocity fields obtained from Gaussian filtering with different filter widths, flow structures within a given bandwidth can be isolated. Energy and dissipation rate spectra are usually computed as a function of spatial or temporal frequency. In this study a constant bandwidth $d k$ in wavenumber space equal to $0.001 \mathrm{~mm}^{-1}$ was used in the band pass calculations. The width of the band-pass is significantly smaller than changes of spectral energy or dissipation rate, which prevents any blurring of the spectra due to convolution with a wide band-pass. Filters sizes for the high-resolution measurements ranged from $0.2 \mathrm{~mm}$ to $12 \mathrm{~mm}$, corresponding to wavenumbers $(k=2 \pi / L)$ from $31.4 \mathrm{~mm}^{-1}$ to $0.52 \mathrm{~mm}^{-1}$, respectively. The quantity $L$ was defined as shown in Equation (6-2), where $f_{1}$ and $f_{2}$ are the filter sizes that bound the bandpass.

$$
L=\frac{1}{2}\left(\frac{1}{f_{1}}+\frac{1}{f_{2}}\right)
$$

The lowest filter size value was extended below $350 \mu \mathrm{m}$ to capture spectral features near the resolution limit. Filters for the low-resolution measurements covered the $1 \mathrm{~mm}$ to $200 \mathrm{~mm}$ spatial domain, corresponding to wavenumbers from $6.3 \mathrm{~mm}^{-1}$ to $0.028 \mathrm{~mm}^{-1}$, respectively. The Gaussian filter was applied to the instantaneous velocity fields for each filter size $k$ and $k+d k$. The filtered velocity fields at $k+d k$ were subtracted from the corresponding fields at $k$ to obtain the band-pass filtered data.

Planar PIV measurements yield two instantaneous velocity components and four components of the velocity gradient tensor. The kinetic energy and dissipation rate can therefore only be quantified in two dimensions. An algorithm was developed and applied to the band-pass filtered velocity fields to calculate the kinetic energy, ke and kinetic 
energy dissipation rate, $\varepsilon$. These quantities have been defined as in Equations (6-3) and $(6-4)$

$k e=\frac{1}{2}\left(u^{2}+v^{2}\right)$

$\varepsilon=\eta\left\{2 u_{x}^{2}+2 v_{y}^{2}+u_{y}^{2}+v_{x}^{2}+u_{y} v_{x}+v_{x} u_{y}\right\}$

In these expressions, $\eta$ represents the kinematic viscosity, $u$ and $v$ the in-plane velocity components, and subscripts $x$ and $y$ denote spatial derivatives. A kinematic viscosity value equal to $2.7 \mathrm{E}-05 \mathrm{~m}^{2} / \mathrm{s}$ was used based on a calculated temperature of 425 $\mathrm{K}$ at $60^{\circ} \mathrm{BTDC}$.

Ensemble-average distributions of the kinetic energy and dissipation rate $\langle k e\rangle$ and $\langle\varepsilon\rangle$, respectively, were computed and then spatially averaged. For the highresolution measurements, a spatial average over the entire FOV was calculated, whereas for the low resolution measurements the spatial average was computed only in an $11 \times 14$ $\mathrm{mm}^{2}$ region at the center of the image (Figure 6-2). This area overlaps with the region investigated with the high-resolution measurements.

The procedure described above was followed twice for each condition listed Table 6-1. It was first applied to the fully post-processed velocity fields, according to the method described in the previous section. In the second case, the velocity fields were post-processed following the same method, but also applying the $3 \times 3$ top-hat filter. Using this filter, the velocity at each $\mathrm{x}_{0}, \mathrm{y}_{\mathrm{o}}$ is calculated as the average of the adjacent points, applying equal weight to all points. Due to the $50 \%$ window overlap used during the final step in the multi-pass processing algorithm, the grid spacing is one half the interrogation window size. The average from the $3 \times 3$ top-hat filter is then computed over a $32 \times 32$ pixel area, which is the size of the final interrogation window. 


\subsection{Kinetic Energy and Dissipation Rate Spectra from Engine Experiments}

Kinetic energy and dissipation rate spectra for high and low swirl engine flows are shown in Figures 6-3 and 6-4. In all graphs, open and filled symbols correspond to values calculated from high- and low-resolution experiments, respectively. Two spectra are shown at each swirl level, obtained from the velocity fields post-processed with and without the $3 \times 3$ top-hat filter. RMS deviations from the spatially averaged kinetic energy and dissipation rate values are shown (as error bars) in all cases. To aid in further explanations, velocity fields processed with the $3 \times 3$ top-hat filter will be referred to as "smoothed velocity fields." The spectra shown in Figure 6-3 a through 6-4 b have not been normalized. However, a variable substitution was needed to make the transformation from space to frequency domain. The steps followed to achieve this transformation are shown in Equations (6-5) through (6-7), where $\Phi_{\mathrm{ij}}$ is the energy spectrum tensor.

$k e \sim \int-\Phi_{i i}(k) d k$

$k \sim \frac{1}{f}$ so $d k \sim-\frac{1}{f^{2}} d f$

$k e \sim \int-\Phi_{i i}\left(\frac{1}{f}\right) \frac{1}{f^{2}} d f$

Based on Equation (6-7), kinetic energy and dissipation rate values should be multiplied by a $1 / \mathrm{f}^{2}$ factor when plotted as a function of the reciprocal of the filter size. In the graphs shown below, these values were divided by $\left\{0.5 *\left(\mathrm{f}_{1}+\mathrm{f}_{2}\right)\right\}^{2}$.

As shown in Figures 6-3 a and b, the kinetic energy spectra calculated from the smoothed velocity fields show a roll off near the spatial resolution limit ( $2 \mathrm{~mm}$ and 350 $\mu \mathrm{m}$ for 32 pixel grid spacing). Kinetic energy values obtained from the non-smoothed velocity fields, on the contrary, peak at the resolution limit for both levels of swirl and spatial resolution. In the low-resolution measurements, the difference in kinetic energy 
between smoothed and non-smoothed velocity fields reaches a maximum at $2 \mathrm{~mm}$, whereas in the high-resolution measurements, this difference peaks between $300 \mu \mathrm{m}$ and $400 \mu \mathrm{m}$. These values correspond to the final interrogation window size $(32 \times 32$ pixels $)$ of the PIV algorithm. These trends are also present for both swirl levels in the dissipation spectra, Figures 6-4a and b. Differences of an order of magnitude in the location of the kinetic energy dissipation rate peak for smoothed and non-smoothed velocity fields can be seen.

Comparison of the non-smoothed, low and high swirl, kinetic energy and dissipation spectra reveals that the spectral peaks do not overlap in the wavenumber range common to both high and low resolution experiments. Rather, the peaks shift towards higher wavenumbers as the resolution of the measurement increases. Regardless of swirl level, the peaks occur at the spatial resolution limit of the measurements. These results lead to question whether the increases in kinetic energy and dissipation rates shown in the spectra are introduced by the PIV evaluation algorithm or are, in fact, intrinsic to the flow. The fixed-displacements experiments conducted by Funk [32] were revisited in this study to answer this question. 

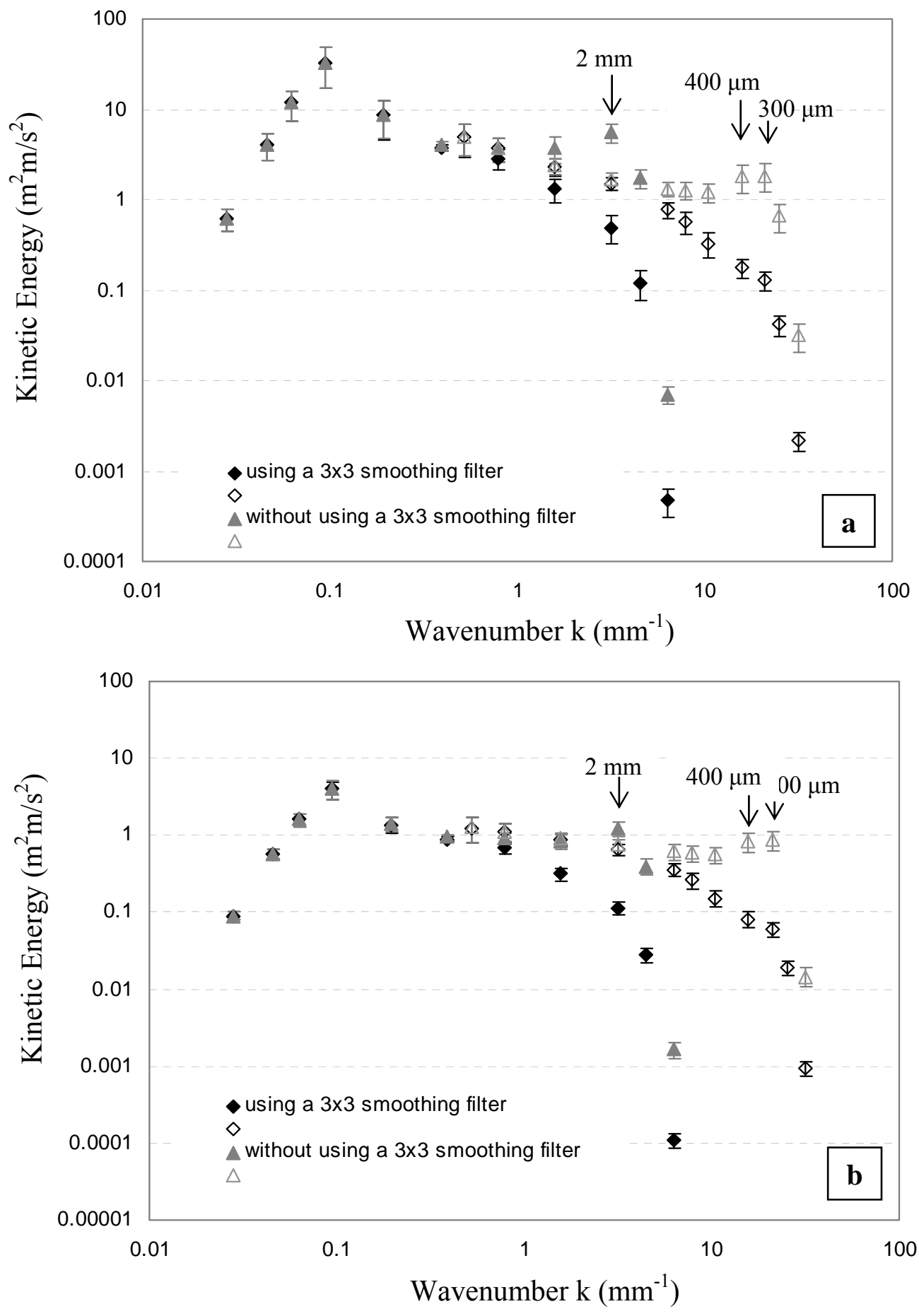

Figure 6-3 Kinetic energy spectra for the high (a) and low (b) swirl flow conditions. The kinetic energy increases near the resolution limit when the $3 \times 3$ smoothing filter is not applied to the velocity fields. 

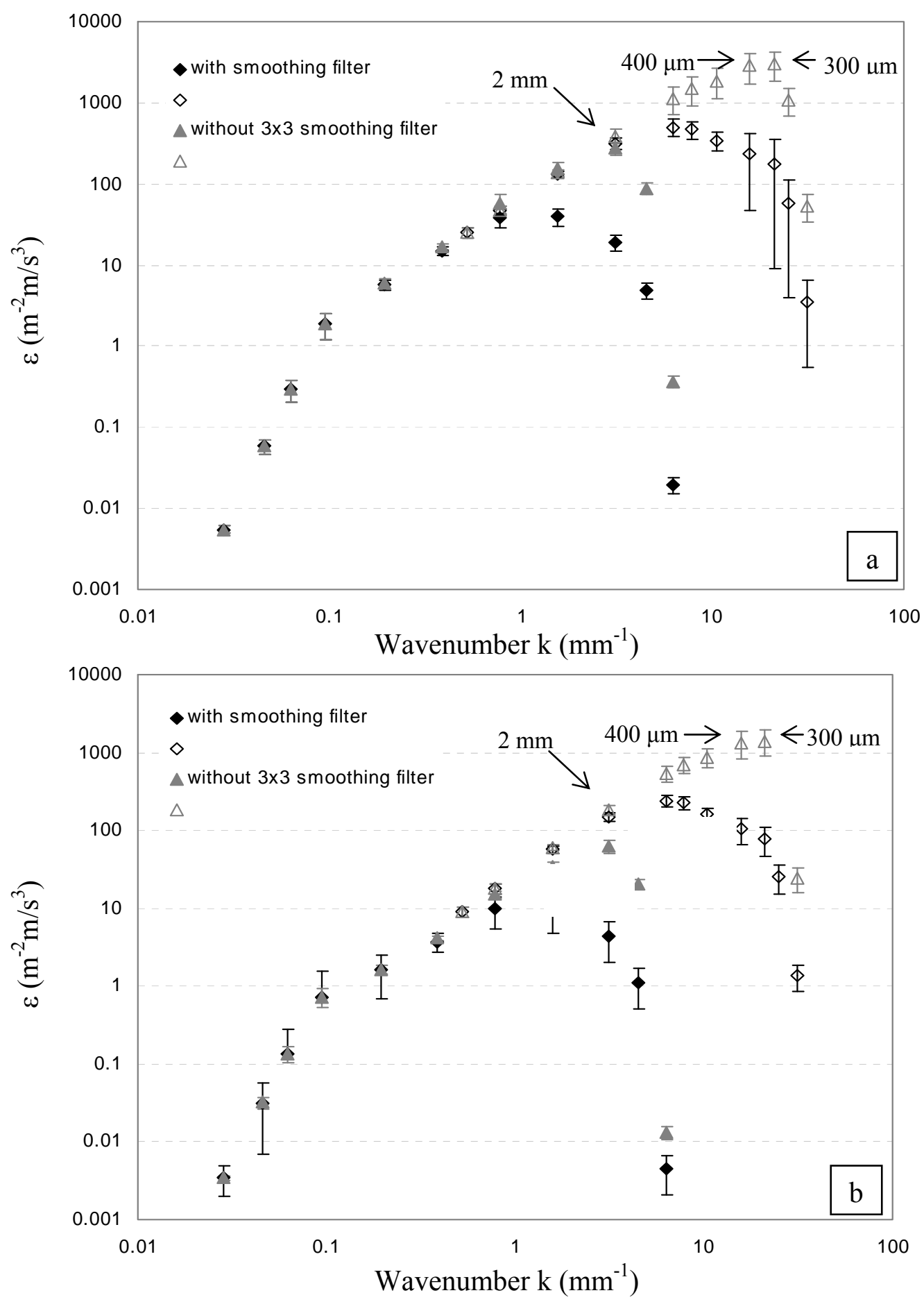

Figure 6-4 Dissipation rate spectra corresponding to the high (a) and low (b) swirl flow conditions. Dissipation rate values increase near the resolution limit when a $3 \times 3$ smoothing filter is not applied to the velocity fields. 


\subsection{Fixed-displacement PIV experiments}

The fixed-displacement control experiment, described in detail in [32], used electro-optical image shifting to reproduce the imaging conditions used in the highresolution PIV engine experiments, while eliminating in-cylinder flow effects on the measurement [30]. A schematic representation is shown in Figure 6-5.

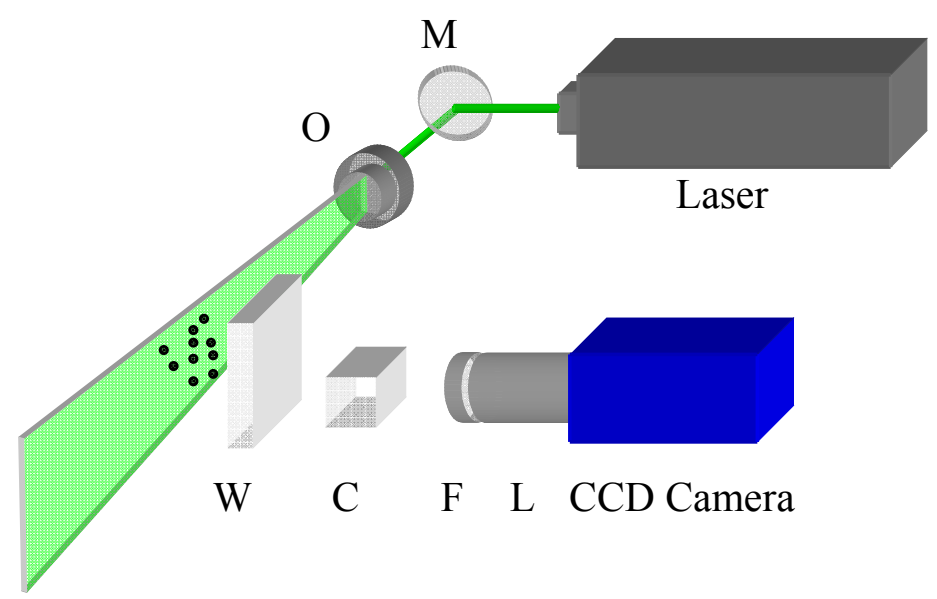

Figure 6-5 Setup for the fixed-displacement experiments. Legend: M: HR 532 mirror, O: light sheet optics, W: piston window, C: calcite crystal, F: narrow-band filter, L:camera lens

The flow was seeded with $1.5 \mu \mathrm{m}$ silicon oil droplets identical to those used in the engine experiments. Simultaneously triggered, cross-polarized laser beams from a dualresonator, frequency-doubled Nd:YAG laser (Continuum Surelite III) illuminated the measurement plane. A quartz window with the same dimensions as the engine flat piston was placed between the object and imaging planes, $4 \mathrm{~mm}$ from the object plane. A $3 \mathrm{~mm}$ calcite crystal, located between the flat window and the recording system was used to create a constant, repeatable shift of the Mie scattered signal. This signal was recorded with the same camera/lens/filter arrangement used for the high-resolution, PIV, engine experiments. The interrogations windows therefore contain images of the same types of particles and at the same number density. 
In the fixed-displacement experiments, the average particle shift exceeded $25 \%$ of the final interrogation window size. This had no detrimental consequences because the light sheets arrive to the FOV simultaneously. With zero delay between laser pulses, the particle shift is solely created by the properties and orientation of the calcite crystal and not by flow motion. To account for the higher than usual shift, the images were autocorrelated in two steps. First, a multi-pass interrogation algorithm with decreasing window size, from $128 \times 128$ to $32 \times 32$ pixels with $50 \%$ overlap, was used. This procedure yielded an initial displacement estimate, which was used as input for the final processing step.

Since the displacement was artificially created by using birefringence and simultaneous laser illumination, uncertainties due to out-of-plane particle losses that may occur in a true three-dimensional flow were eliminated. By replicating the details of the engine experimental setup (e.g. f-stop and magnification), errors introduced by optical aberrations and particle focus were also reproduced. As noted in [30], the calcite crystal causes additional aberrations. Nonetheless, this fixed-particle-displacement experiment isolated uncertainties introduced by the PIV processing algorithm.

\subsubsection{Spectra from fixed-displacement experiments}

The two-dimensional energy spectra for the artificially created fixed-displacement fields are presented in Figure 6-6. Triangle and diamond symbols have been used to represent data corresponding to non-smoothed and smoothed displacement fields, respectively, at each filter size. These data have been processed using the same Gaussian filtering technique (Equation 6-1) and kinetic energy algorithm that were applied to the engine velocity data. However, in the absence of a laser-pulse time delay, the particle displacement fields are caused by the birefringence alone. For this reason, the spectra are shown in arbitrary units. 


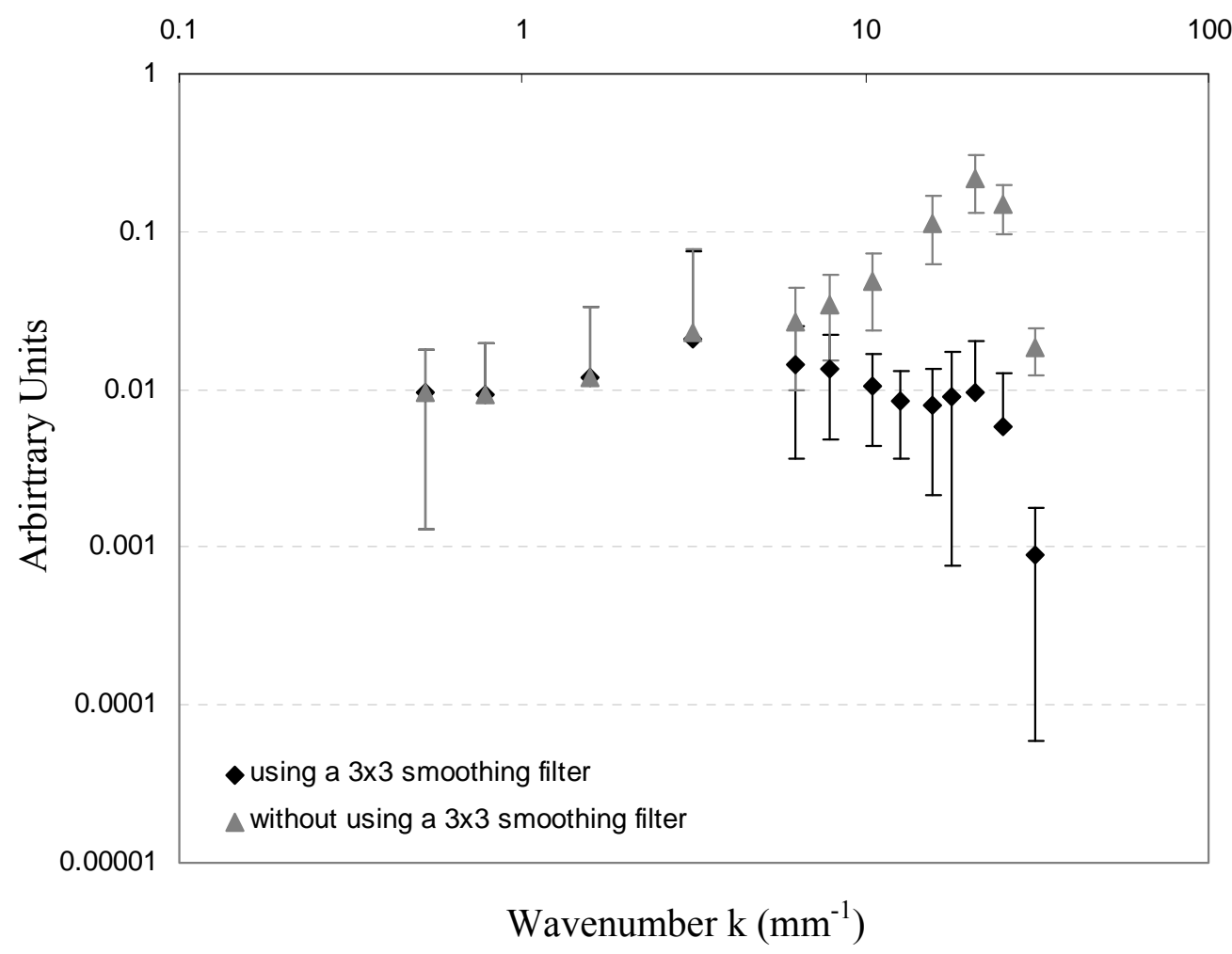

Figure 6-6 Spectra obtained from band-pass filtering the artificially generated flow field. The values were calculated with the same routine used to obtain the kinetic energy spectrum of the engine flows. The peak occurs at $300 \mu \mathrm{m}$.

Spatial-RMS variations in the ensemble mean displacement field, shown as error bars in Figure 6-6, arise from (1) expected systematic variations introduced by the birefringent crystal, (2) the sub-pixel correlation-peak detection of real images, which have both systematic and random aberrations, and (3) possibly noise introduced by random sources (e.g. shot noise). Due to the absence of flow structure, the resulting spectra are expected to be flat (i.e. invariant with filter size) if only white noise is present. Within experimental uncertainty, this is the case for the spectra calculated from smoothed displacement fields (see Figure 6-6). The spectrum calculated from the non-smoothed displacement fields, however, diverges from the expected flat profile and peaks at 300 $\mu \mathrm{m}$, the spatial resolution limit of the measurements. 


\subsection{Discussion}

Results from the fixed-displacement and engine experiments are consistent in that in both cases, a peak in the spectra exists at small scales, on the order of the interrogation window size. Since no flow motion is present for the fixed-displacement experiments, it can be concluded these peaks arise as a consequence of noise introduced during the PIV interrogation process. This follows from two main observations. First, in both cases the noise enters the spectra at large wavenumbers (smallest length scales), where uncertainties in detecting the correlation peak are on the same order as the particle displacements. Second as shown by the engine flow spectra, the peaks follow the resolution limit of the PIV experiments. The presence of noise peaks at the resolution limit of the measurement implies that the smallest spatial scales (largest wavenumbers) that can be resolved are larger than the size of the final interrogation window. It also suggests that to accurately quantify the kinetic energy and dissipation rate at the inner scales of IC engine flows, the spatial resolution of the PIV measurement should be higher than required to resolve the smallest flow scales.

Nonetheless, it is important to realize that this noise causes an overestimation of the kinetic energy and dissipation rate. Furthermore, the theory of homogeneous, isotropic turbulence predicts a peak in the dissipation spectra at eight times the Kolmogorov scale. Ambiguity in the location of the dissipation spectral peak increases the uncertainty in calculating the Kolmogorov scale. Granted, kinetic energy and dissipation rate values calculated from planar PIV measurements underestimate the true values because not all components of the velocity and its spatial derivatives can be measured. Noise however, could also corrupt the spectra at the resolution limit for threedimensional measurements. Being aware of the extent to which the spectra is corrupted by noise at the smallest scales is important to draw meaningful conclusions from 
experimental data and also when using data for comparison with RANS- and LES-based models.

Comparison of spectra obtained from smoothed and non-smoothed velocity fields, reveals that a $3 \times 3$ top-hat filter effectively removes the enhanced values at large wavenumbers. Since the particle displacements are artificially created for the fixeddisplacement experiments, the averaging process in this case filters the high-frequency, random noise at the resolution limit of the measurements. In real flows however, the filter does not separate noise from flow contributions. Thus the kinetic energy and dissipation rate at scales approaching the resolution limit of the measurements is also misrepresented in this case.

The three-point Gaussian function has been argued to yield more accurate estimates of particle-peaks than linear or parabolic interpolation [45,46], although differences in the performance of peak estimators is less pronounced if the particles are well sampled (i.e. for particle sizes greater than 2 pixels). The cross-correlation algorithm used in this work to calculate particle displacements implements the Gaussian peak estimators. Furthermore, the mean particle image diameter is approximately 4 pixels. Real particle images however, are not ideal. As discussed in Chapter 2, both systematic and random errors are introduced due to non-ideal optical elements, noise in the light field and imaging system. Uncertainties can be introduced by the Gaussian peak-fitting approach, which approximates the ideal Airy function description of a particle image by a Gaussian. If the noise is then due to uncertainties in peak detection, noise reduction would require improvements in optical components and higher resolution of the imaging system. 


\subsection{Conclusions}

Results from planar digital PIV measurements performed in an optical engine in a horizontal plane $4 \mathrm{~mm}$ above the piston surface were used for this investigation. These experiments achieved a spatial dynamic range from $55 \mathrm{~mm}$ down to $350 \mu \mathrm{m}$ using two experimental setups. These low- and high-resolution setups employed magnifications of 0.11 and 0.62 , respectively, and appropriately scaled light sheet thicknesses. Vector fields from 300 engine cycles for two different swirl levels were determined for each magnification. Kinetic energy and dissipation rate spectra of the total velocity fields were calculated by spatially filtering the data with a Gaussian filter approach that ensured constant wavenumber.

Comparisons between low- and high-resolution measurements show an abrupt increase in both energy and dissipation rate spectra at the spatial resolution limit of the measurements. This increase can be attributed to noise introduced by the PIV interrogation algorithm. Three pieces of evidence support this conclusion: (1) the peaks are not coincident at the same scales for high- and low- resolution measurements, (2) they occur at the spatial resolution limit of each setting, (3) they can be found in spectra calculated from fixed-displacement experiments performed with electro-optical image shifting.

Results have shown that a $3 \times 3$ spatial averaging filter applied to the individual vector fields removes the peaks in the spectra. However, the use of the traditional $3 \times 3$ filter will also attenuate kinetic energy and dissipation rate values at the scales of the PIV resolution. The presence of the spectral noise at the resolution limit of the PIV measurements creates difficulties in quantifying the kinetic energy and dissipation rate of IC engine flows at large wavenumbers near the interrogation window size. Reducing the noise at these scales is necessary even at the expense of overall spatial resolution. 


\section{CHAPTER 7}

\section{HIGH-SPEED UV PIV USING A TWO LASER-ONE CAMERA SYSTEM}

The experimental setup and results from a high-speed PIV technique using a single-laser, single-camera arrangement and $355 \mathrm{~nm}$ excitation wavelength have been described in Chapter 4. This technique was limited by the single, fixed laser pulse frequency, which did not allow any flexibility in adjusting the delay between laser pulses. It is important however, that the laser pulse delay be optimized based on the flow velocity, which in turn varies with engine speed. In PIV experiments, laser power is always a valuable resource. The output laser power decreases with increasing pulse frequency and decreasing output wavelength. Consequently, in the single-laser-camera experiment, the maximum frequency at which the laser could be operated $(16 \mathrm{kHz})$ was selected as a compromise to achieve adequate laser pulse delay and output laser power. To meet these requirements, the engine was run at 600 RPM. Extending the application of this technique to 2000 RPM was one of the original goals. This chapter describes the necessary modifications to the original technique and the application of high-speed UV PIV to measure two-dimensional velocity fields in a stratified SIDI engine at 2000 RPM.

\subsection{Experimental Equipment and Setup}

A second frequency-tripled high-speed laser (Quantronix Hawk II) was added to gain flexibility in adjusting the delay between laser pulses. The laser power output, rated as $5 \mathrm{~W}$ at $6 \mathrm{kHz}$, is equivalent to approximately $0.8 \mathrm{~mJ}$ per laser pulse. The presence of a second laser required the addition of beam-combining optics and a method to monitor 
light sheet alignment and overlap. The modified experimental setup is illustrated in Figure 7-1. A $1 / 2$ waveplate was placed near the output of one of the lasers (laser A) to rotate the polarization of the original, vertically-polarized beam. A HR 355/HT 532 mirror (M1) reflected the beam from the second laser (laser B). The beams were recombined using a UV-coated polarizing beam-splitting cube (PBS) and then reflected off a second HR 355/HT 532 mirror (M2) along the path to the engine.

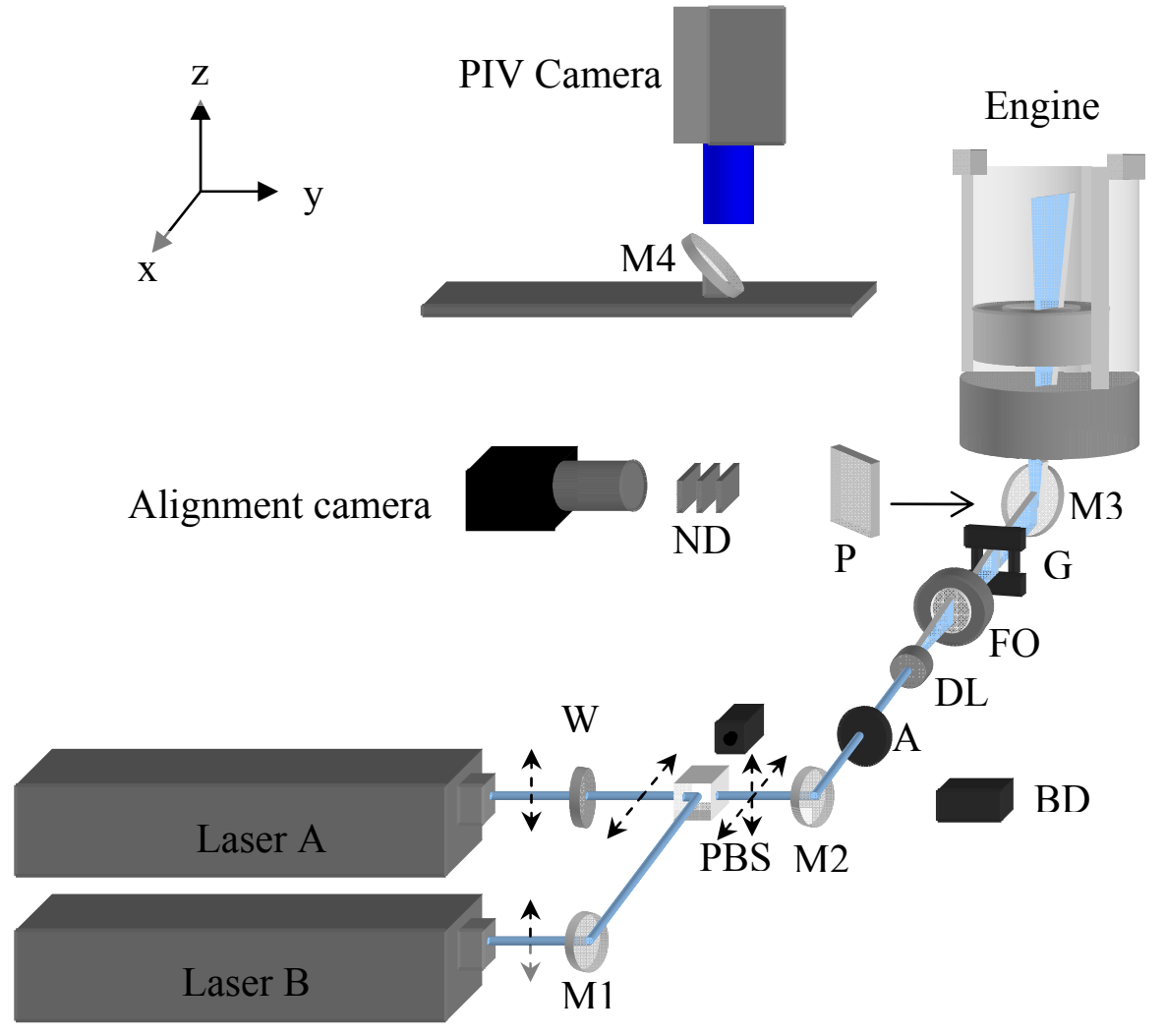

Figure 7-1 Experimental setup for the dual-laser single-camera high speed UV PIV setup. Legend:-M1, M2: HR 355 HT 532 mirrors, W: 1/2 waveplate, PBS: polarizing beamsplitting cube, BD: beam dump, A: aperture, DL: diverging lens, FO: focusing optics, G: gate, M3: $45^{\circ} \mathrm{UV}$-enhanced mirror, ND: neutral density filters, P: glass plate, M4: HR 355 mirror. Dotted arrows indicate polarization of the output beams.

Using a $-20 \mathrm{~mm}$ cylindrical plano-concave lens (DL), the beams were diverged into two coincident light sheets, which then passed through a variable focal lens objective containing two spherical lenses (FO). The focus was set to reduce the thickness of the light sheets to $1 \mathrm{~mm}$ at the measurement plane. The distance between DL and FO was 
adjusted to achieve $15 \mathrm{~mm}$ widths. The light sheets reflected off a 45-degree UVenhanced mirror positioned at the entrance to the engine and entered the cylinder through the transparent (quartz) piston top. As in the previous PIV experiments, silicon oildroplets ( $1 \mu \mathrm{m}$ nominal diameter) were used as seeding. The seeding particles entered the engine after mixing with air in the intake plenum. The scattered signal was reflected off a 4-inch diameter, $45^{\circ} \mathrm{UV}$-coated mirror. A high-speed CMOS camera (Phantom 7.1) was positioned parallel to the cylinder axis (refer to Figure 7-1) and fitted with a 105 $\mathrm{mm} \mathrm{f/4.5} \mathrm{UV} \mathrm{lens} \mathrm{(Coastal} \mathrm{Optics} \mathrm{SLR).} \mathrm{The} \mathrm{minimum} \mathrm{f-stop} \mathrm{setting} \mathrm{(f/4.5)} \mathrm{was}$ selected to maximize light collection. The camera orientation with respect to the imaging plane was chosen based on two considerations. First, the reflectivity of the $45^{\circ} \mathrm{UV}$ mirror is superior to the transmissivity of the $355 \mathrm{~nm}$ narrow-band filter previously used in the single-laser, single-camera arrangement (compare Figure 7-2 with Figure 7-3). Second, this arrangement facilitates the location of the high-speed biacetyl-PLIF equipment for future simultaneous measurements of the flow and equivalence ratio distribution in the SIDI engine (Figure 7-1). Since the PLIF hardware includes a second camera, a GG-420 filter and an image intensifier, setup and alignment becomes less cumbersome if the multi-component PLIF system is located on the horizontal (x-y) plane. 


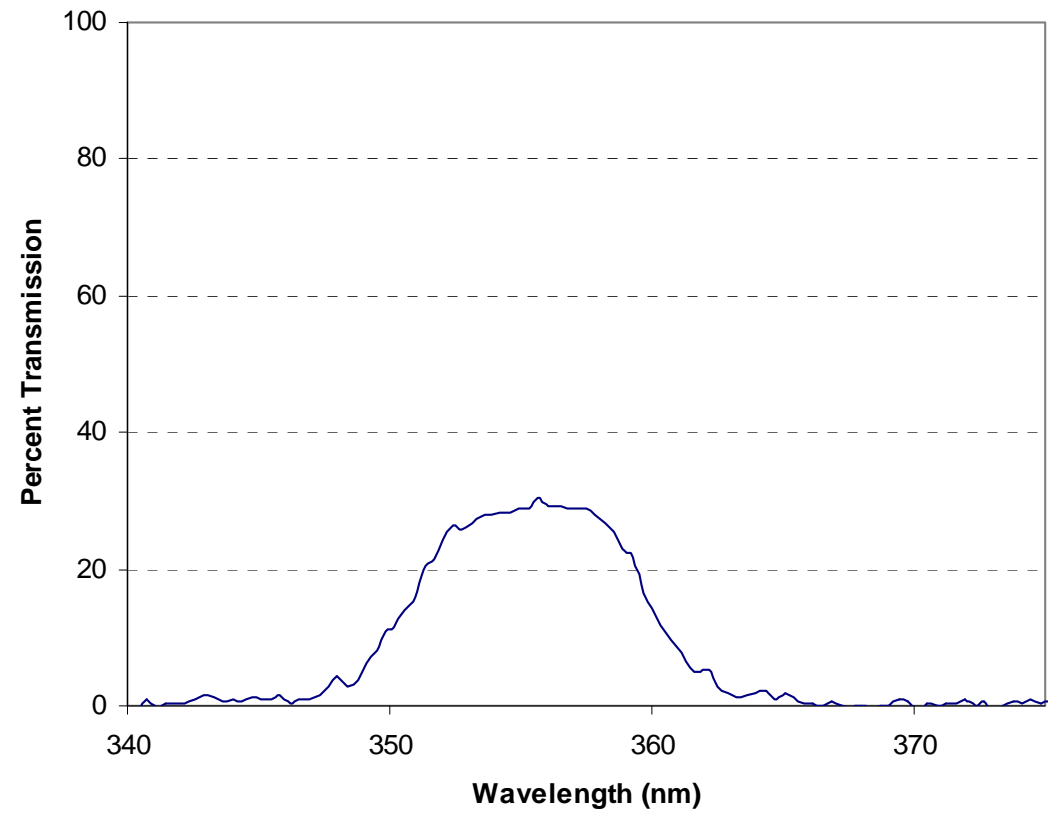

Figure 7-2 Transmissivity of the 355 narrow-band filter used in the single-laser, singlecamera high-speed PIV setup, showing that the incoming signal is attenuated by approximately $70 \%$ at $355 \mathrm{~nm}$.

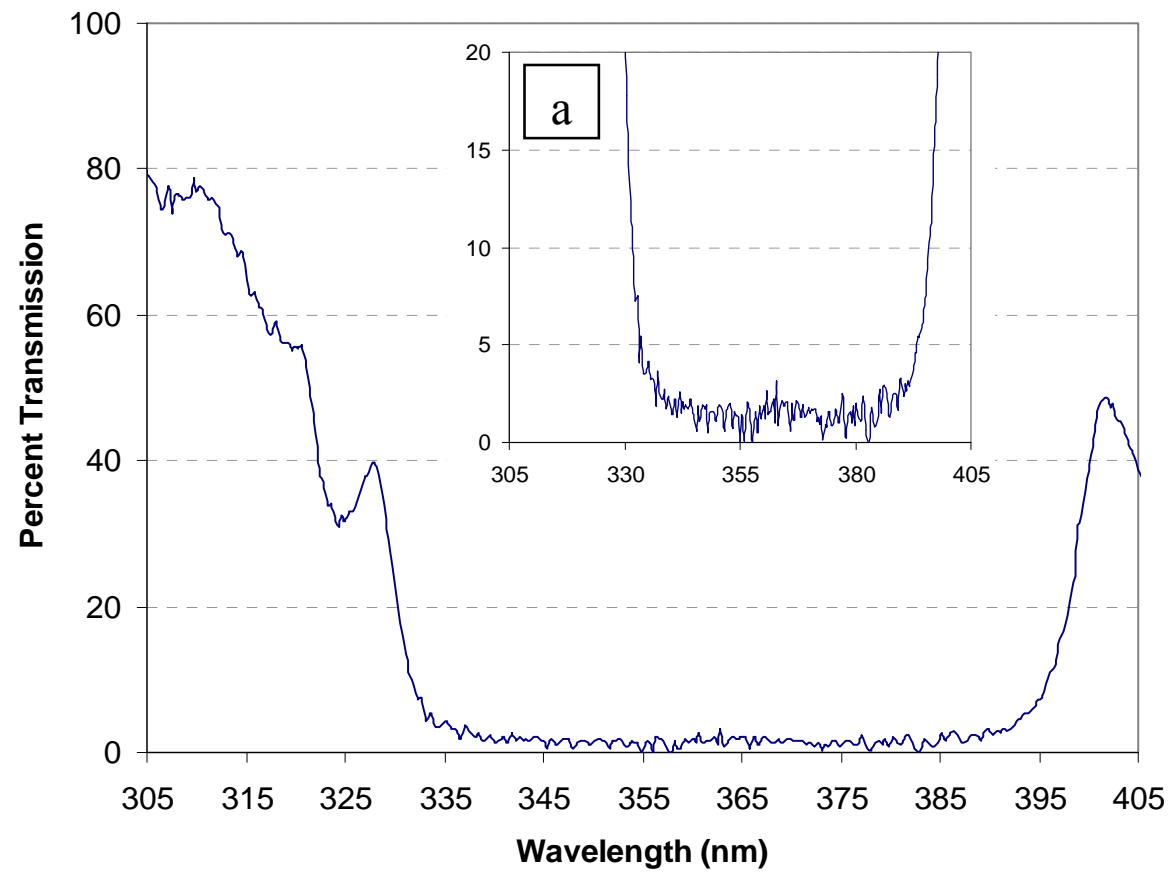

Figure 7-3 Transmission curve for to the $45^{\circ} \mathrm{UV}$-coated mirror, showing (see also inset with expanded ordinate) between $98 \%$ and $99 \%$ reflectivity at $355 \mathrm{~nm}$. 
The PIV imaging system was located as close to the engine cylinder as hardware and space constraints allowed, while maintaining comparable spatial resolution to previous PIV experiments. The selected location yielded a 0.53 magnification and 1.4 $\mathrm{mm}$ in-plane spatial resolution, based on a $32 \times 32$ final interrogation window size. In Figure 7-5, the outline of the dish piston has been superimposed on the top view of the cylinder head to illustrate the dimensions of the field of view (FOV) in the $x-z$ plane and its orientation relative to the spark plug. As part of the experimental procedure, images of a calibration target (one millimeter resolution) were recorded at the measurement plane. These images would subsequently be used to determine the pixel-to-millimeter scaling parameters for the velocity calculations.

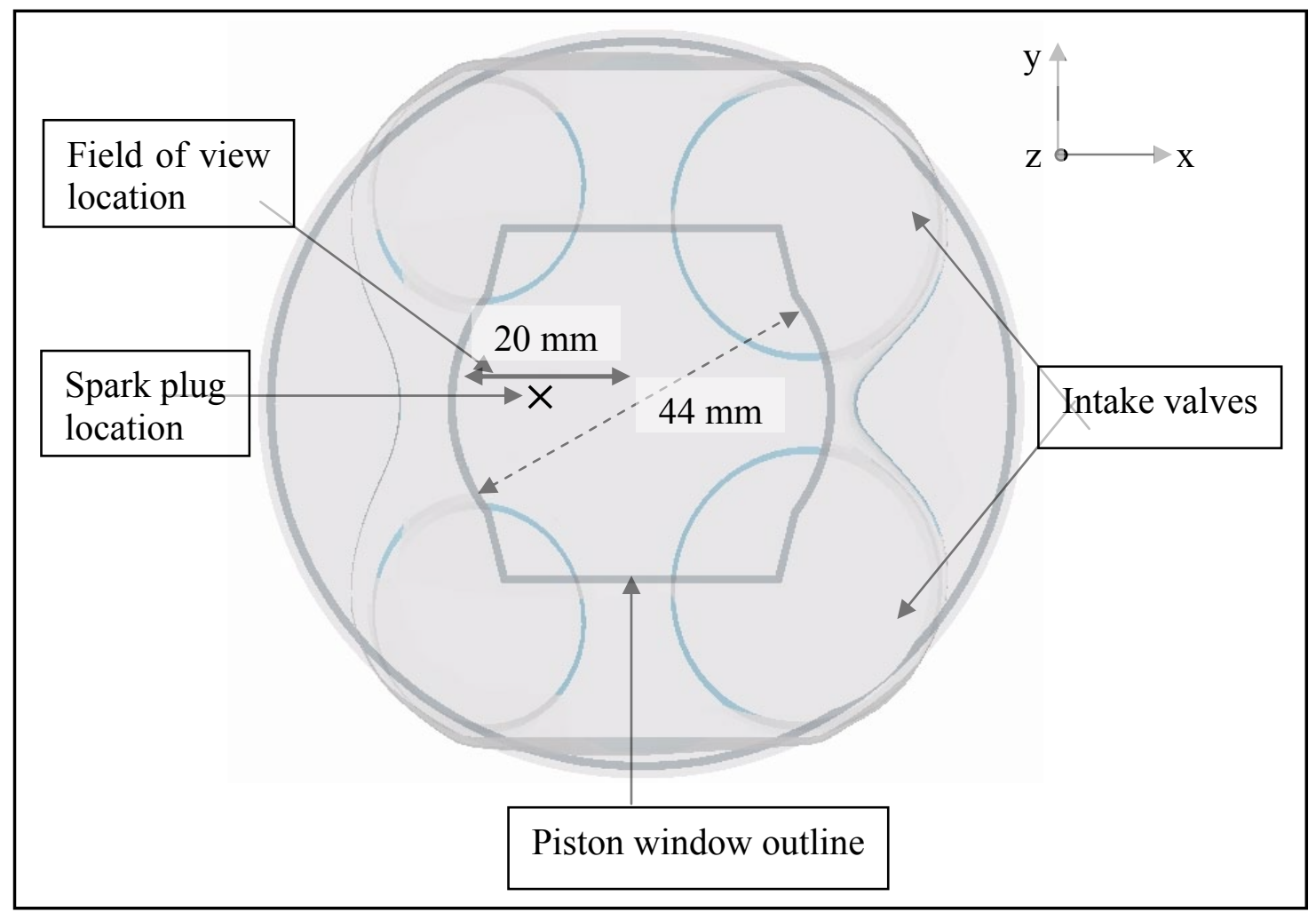

Figure 7-4 Top view of the engine head and piston bowl showing the location of the field of view used in the dual-laser, single-camera high-speed UV PIV measurements 
As discussed in Section 2.2, experimental investigations on the accuracy and precision of PIV measurements when imaging through a curved surface suggest that the FOV should be restricted to the center $66 \mathrm{~mm}$ to minimize the uncertainty in detecting particle displacements. A second issue related to cylinder curvature arises from the sensitivity of light transmission to polarization. The angle of incidence of the scattered light rays increases with cylinder curvature. At large angles of incidence, s-polarized (TM polarization) rays are transmitted more than p-polarized (TE polarization) rays. In the current setup, laser A and B are p-polarized and s-polarized, respectively. Based on this, the transmitted scattered signal corresponding to laser B would be of higher intensity at large angles of incidence. This could cause pairing losses and affect particle detectability during the cross-correlation process. The decrease in the percentage of valid vectors near the cylinder wall shown by Reuss et al. [see Figure 8 in 31] can be partially attributed to this phenomenon. Specifically, the detectability remained above $95 \%$ at $\mathrm{x}$ locations at or below $25 \mathrm{~mm}$ from the cylinder axis. In the current setup, the FOV is constrained within $20 \mathrm{~mm}$ from the cylinder axis to avoid the unwanted effects associated with pronounced cylinder curvature just described.

Since the maximum aperture setting was selected, the correspondingly short depth of field created a challenge in achieving adequate focus of the tracer particles. To properly focus the tracer particles, the intake and exhaust valves were manually opened after disconnecting the timing belt. With the engine cylinder in place, seeding particles were introduced in the combustion chamber. Next, the camera lens was adjusted to achieve a rough image focus and was then fixed in place. A micrometer-controlled translation stage was used to vary the camera location in the z-direction (refer to Figure 7-1) until the focal plane was found.

Achieving and maintaining adequate light sheet alignment and overlap from shotto-shot became another relevant challenge, due specifically to the short depth of field associated with the f-stop selected and due to vibrations incurred during engine operation. 
Beam alignment was verified, first by ensuring that beam A burned at the same location of a target placed in front of and behind the entrance to the engine. Next, M1 was used to adjust the $\mathrm{y}$ and $\mathrm{z}$ locations of beam B and ensure overlap with respect to the reference beam (beam A). Light sheet alignment and overlap at the imaging plane was verified by adding a second (CCD) camera to the setup, also schematically shown in Figure 7-1. A glass plate (P) was placed just before the $45^{\circ}$ engine mirror to reflect a portion of the light sheets. The reflections were imaged by a CCD camera positioned to match the length of the beam path from the glass plate to the imaging plane. The light sheet images were displayed on a television monitor. Neutral-density filters (ND) were placed before the alignment camera to attenuate the intensity of the reflected light. This helped discern light intensity gradients in the displayed light sheet images to verify the overlap.

Achieving and maintaining proper light sheet alignment and particle focus is especially important for PIV measurements in engines due to vibrations incurred during equipment operation. Consequently, the robustness of the setup (e.g. secured mounts, translation stages, and cleanliness of optical components), light sheet alignment and overlap was verified before each engine run.

\subsection{Triggering Scheme}

A schematic of the main components of the triggering system is shown in Figure 7-5. Some devices have been omitted for clarity. Triggering and synchronization of the two-laser, single-camera system with the engine and main cycle events (i.e. fuelinjection, ignition, etc) were accomplished using a signal generator (Stanford Research Systems DG 535). The signal generator received a one-per-crank angle (12 kHz) signal from the engine crank shaft encoder and produced four $12 \mathrm{kHz}$ outputs synchronized with the crankshaft. 


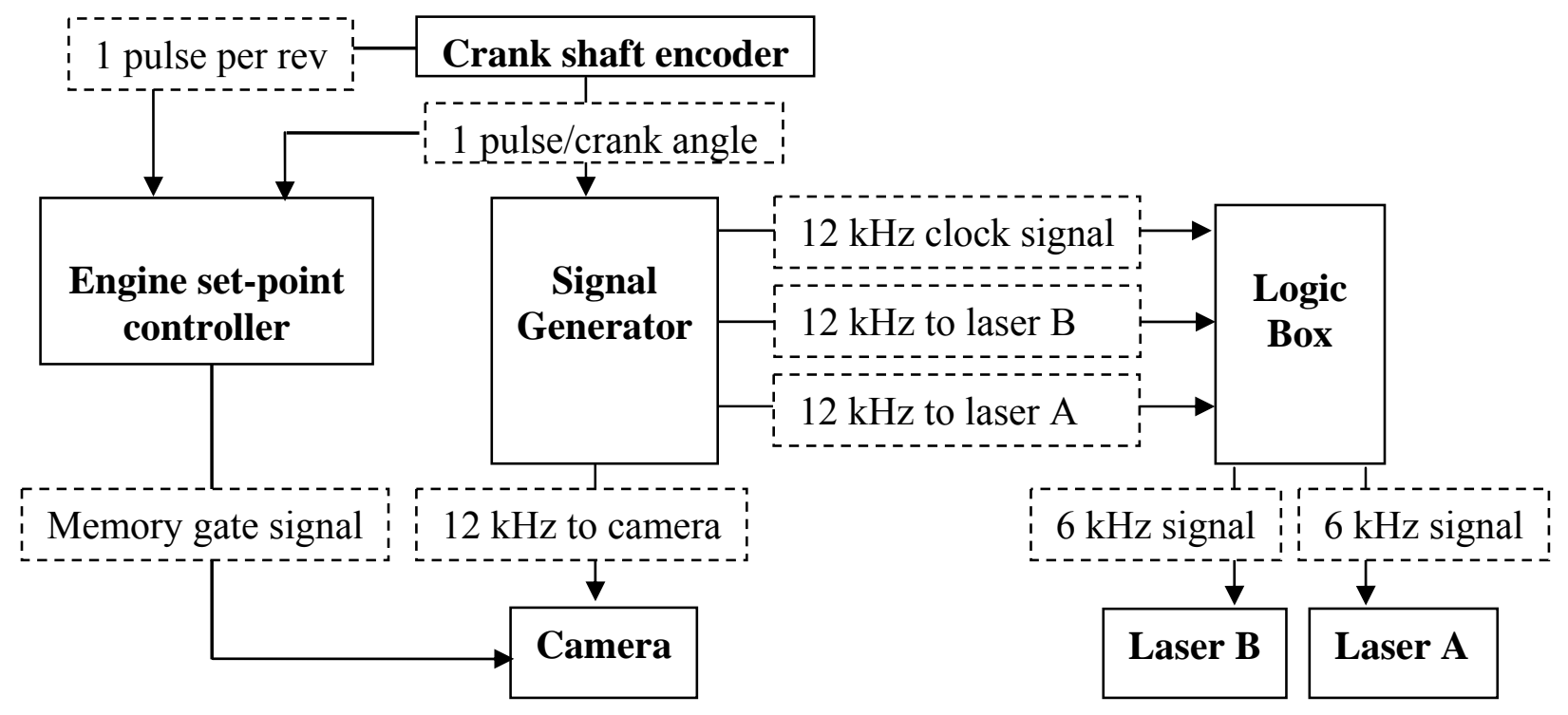

Figure 7-5 Main equipment and signals used to synchronize the high-speed lasers and cameras used in the UV PIV experiment. Signals are shown in dotted-outline text boxes.

One of these signals directly triggered the camera. The remaining three were routed to a logic box that halved the original frequency to $6 \mathrm{kHz}$. Each laser response to the input trigger signals was verified with a photodiode, placed in front of the light sheetalignment camera shown in Figure 7-1. Triggering delays between the camera and lasers were adjusted using the signal generator and constantly monitored with an oscilloscope.

To ensure synchronization of the acquisition system with the main cycle events and maximize the available camera memory space, the camera memory-gate function was employed. This option prevents recording images continuously, which at the current acquisition rate would fill the camera memory in approximately 50 cycles. Instead, image acquisition was triggered only for the portion of the engine cycle for which the PIV parameters (e.g. time delay, seeding) had been optimized. This also permitted increasing the number of cycles over which images are recorded by an order of magnitude.

Signals from the crankshaft and camshaft encoders are routed to the engine setpoint controller, which outputs fuel injection, ignition signals to trigger the main engine 
cycle events. To implement the camera memory-gate function, an additional output signal was used to initiate camera recording at specific crank angles. The recording length can be specified in either "real time" (ms) or crank angles. Since the crankshaft encoder does not produce a constant $12 \mathrm{kHz}$ signal due to cycle-to-cycle fluctuations in engine speed, the recording duration was specified in crank angles for all experiments. This approach ensured correct synchronization of the camera and lasers (within $\pm 5 \mu \mathrm{s}$ ) with the piston location over the course of the measurements.

The CMOS camera was triggered at $12 \mathrm{kHz}$ in frame straddling mode with its frequency-dependent, variable exposure time set at its maximum $(81.3 \mu \mathrm{s})$. At $12 \mathrm{kHz}$, the effective image format is $480 \times 480$ pixels. Each laser was triggered at $6 \mathrm{kHz}$, as illustrated in Figure (Figure 7-6). With this triggering scheme, two images at every other crank angle can be recorded at 2000 RPM engine speed. The image pair was then crosscorrelated to obtain one velocity field at every other crank angle.

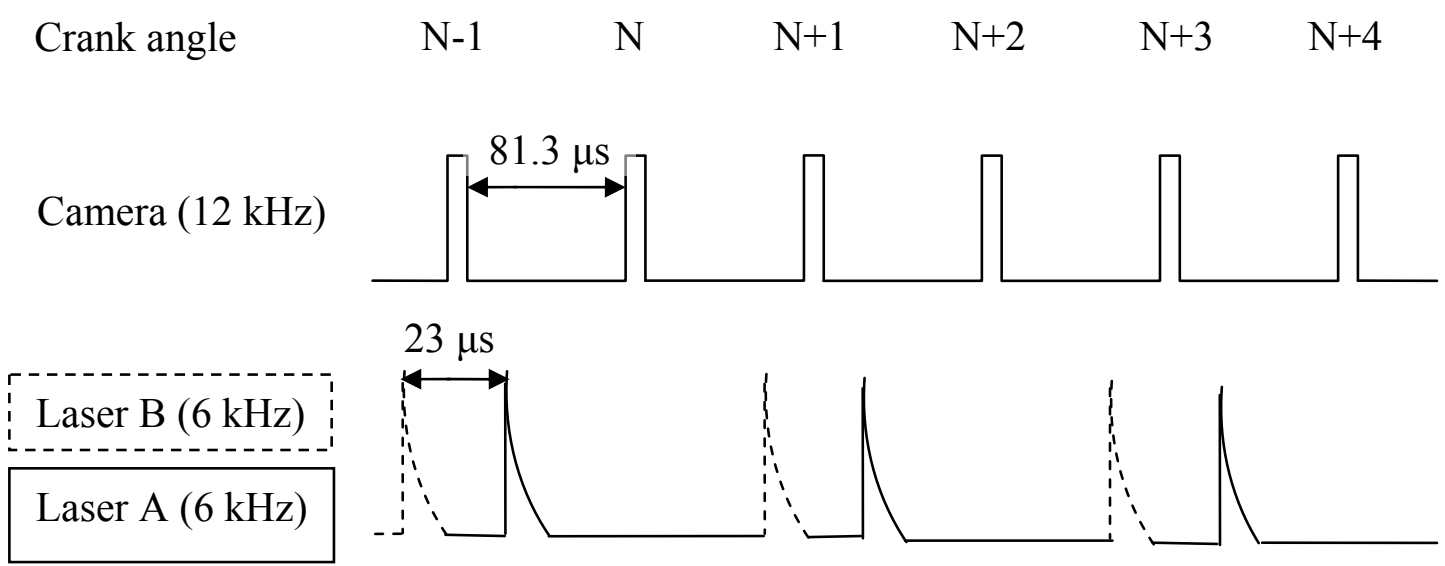

Figure 7-6 Triggering schematic for the dual-laser, single-camera UV PIV experiment

Achieving crank angle resolution would have required doubling the laser and camera frequencies. Doubling the laser frequency to $12 \mathrm{kHz}$ would further reduce the output power and hence the Mie-scattered signal. Doubling the camera frequency would reduce the camera image format and consequently the spatial resolution at the same magnification. To maintain the same spatial resolution, the magnification would have to 
be increased, further reducing the depth of field. This would detrimentally affect particle focus, which has been experimentally shown to introduce up to $4 \%$ RMS error in particle detectability [30]. These considerations led to selecting the triggering scheme shown in Figure 7-6.

Results from two experiments will be presented. In both, the recording was initiated at $83{ }^{\circ} \mathrm{BTDC}$ in the compression stroke and lasted until $27^{\circ} \mathrm{BTDC}$ at the rate of two images every other crank angle (Figure 7-6). The recording was paused and repeated for 500 consecutive cycles. Also in both cases the engine was run at 2000 RPM and the intake pressure and temperature were regulated to $95 \mathrm{kPa}$ and $45^{\circ} \mathrm{C}$, respectively. A summary of test parameters is shown in Table 7-1.

Table 7-1 Experimental parameters for the high-speed dual-laser UV PIV setup

\begin{tabular}{|l|r|}
\hline \multicolumn{1}{|c|}{ Parameter } & \multicolumn{1}{c|}{ Value } \\
\hline Light sheet width & $15 \mathrm{~mm}$ \\
\hline Light sheet thickness & $1 \mathrm{~mm}$ \\
\hline Field of view & $20 \mathrm{~mm}^{2}$ \\
\hline Magnification & 0.53 \\
\hline f-number & 4.5 \\
\hline Depth of field & $2 \mathrm{~mm}$ \\
\hline In-plane spatial resolution & $1.4 \mathrm{~mm}$ \\
\hline Dynamic velocity range & $66: 1$ \\
\hline Maximum resolvable velocities & $15 \mathrm{~m} / \mathrm{s}$ \\
\hline Engine speed & $2000 \mathrm{RPM}$ \\
\hline Intake temperature & $45^{\circ} \mathrm{C}$ \\
\hline Intake pressure & $95 \mathrm{kPa}$ \\
\hline
\end{tabular}

For the first experiment, the engine was run under motored conditions. The fuelinjection and ignition drivers remained deactivated. For the second experiment the engine speed was first increased to 2000 RPM by regulating the dynamometer speed. Once the speed became stable, the injection and ignition drivers were activated and data 
acquisition started. In subsequent discussions, the first and second experiments will be referred to as motored-only and fired experiments, respectively.

\subsection{Data Processing}

The recorded movies were converted to image files, imported into DaVis 7.1 and reorganized in double-frame images for further processing. Scaling parameters determined using a millimeter-resolution calibration target were applied to a scale the images. The resulting files were then reorganized by crank angle. This was done in anticipation of the need to employ different masks during vector field computations due to variations in the piston location and spray penetration near top-dead-center.

In both the motored-only and fired runs, unseeded and non-illuminated areas in the field of view were excluded from processing. These areas (e.g. the spark plug electrode location) remain constant. Consequently a fixed, geometric mask was used at each crank angle. For the fired experiment, an additional dynamic algorithmic mask was created to exclude the areas covered by the fuel spray jets. This was a challenging task due to variability in the compactness of the spray, especially after $41^{\circ} \mathrm{BTDC}$.

A high-pass filter (15 pixels) was used to reduce low-frequency background noise recorded in the images. The double-frame images were then cross-correlated using an FFT-based algorithm and decreasing interrogation widow sizes $(128$ x 128, 64 x 64, 32 x 32 pixels) with $50 \%$ overlap. A standard deviation operator was used to detect and replace spurious vectors by the mean of the adjacent four vectors. The resulting velocity fields were then smoothed using a 3 x 3 top-hat filter.

\subsection{Data Validation}

Two metrics often used to evaluate the quality of PIV data are (1) the ratio of signal to noise peaks in the correlation map, also referred to as the quality (Q) factor and 
(2) the percentage of vectors retained after post-processing. These quantities are shown in Figure 7-7 for approximately 500 instantaneous velocity fields at the crank angles selected for these experiments. Results between $83{ }^{\circ} \mathrm{BTDC}$ and $47{ }^{\circ} \mathrm{BTDC}$ corresponding to the fired experiment are shown in Figures 7-7 a and b. Figures 7-7 c and d show motored-only experiment results from $83{ }^{\circ} \mathrm{BTDC}$ to $41{ }^{\circ} \mathrm{BTDC}$, whereas Figure 7-7 e encompasses the crank angles up to $31^{\circ} \mathrm{BTDC}$.

The fuel injection event, triggered at $57{ }^{\circ} \mathrm{BTDC}$, is expected to last at least until $40{ }^{\circ}$ BTDC. As will be shown in the next section, residual spray droplets are found even after ignition has been triggered at $34{ }^{\circ}$ BTDC. During most of the fuel injection event the spray jets extend across the FOV, notably reducing the area available for velocity computations. This accounts for the difference in the lower crank angle limit $\left(31^{\circ} \mathrm{BTDC}\right.$ vs. $47^{\circ}$ BTDC) between motored and fired cases for the results presented in Figure 7-7.

Specific crank angles ranges have been plotted separately to illustrate differences in the quality of the data. When comparing Figure 7-7 a through d, it can be seen that for both the motored and fired experiments, the average Q-factor remained between 1.8 and 2. The percentage of first-choice vectors, however, is $10 \%$ to $15 \%$ lower between 83 ${ }^{\circ} \mathrm{BTDC}$ and $71^{\circ} \mathrm{BTDC}$ than between $69{ }^{\circ} \mathrm{BTDC}$ and $47^{\circ} \mathrm{BTDC}$ (or $41^{\circ} \mathrm{BTDC}$ in the motored case). These differences might be partially attributed to seeding density changes as the cylinder volume changes during the compression stroke. As noted earlier, a single setting for the seeding generator will not provide optimum seeding density over a broad range of crank angles. Consequently, if the seeder setting is optimized for crank angles say, near $60{ }^{\circ} \mathrm{BTDC}$, the volume will be under-seeded near $90{ }^{\circ} \mathrm{BTDC}$ and over-seeded near TDC.

This observation is supported by the upwards shift in the data as the crank angles approach TDC, shown in Figure 7-7 b and $\mathrm{d}$ for the fired and motored experiments, respectively. The percentage of first-choice vectors is higher for the motored case than for the fired experiment (compare Figures 7-7 $\mathrm{b}$ and $\mathrm{d}$ ). These differences were also 
found in the preliminary experiments presented in Chapter 3 (see Table 3-4). A likely cause is the more rapid fouling of the quartz cylinder during fired-engine runs. Based on the results just presented, data obtained between $83{ }^{\circ} \mathrm{BTDC}$ and $71{ }^{\circ} \mathrm{BTDC}$ were not used in subsequent calculations. Between $69^{\circ} \mathrm{BTDC}$ and $47^{\circ} \mathrm{BTDC}$ (or $31^{\circ} \mathrm{BTDC}$ for the motored case), only velocity data with validation rates above $80 \%$ and Q-factors at or above 1.8 were considered. 

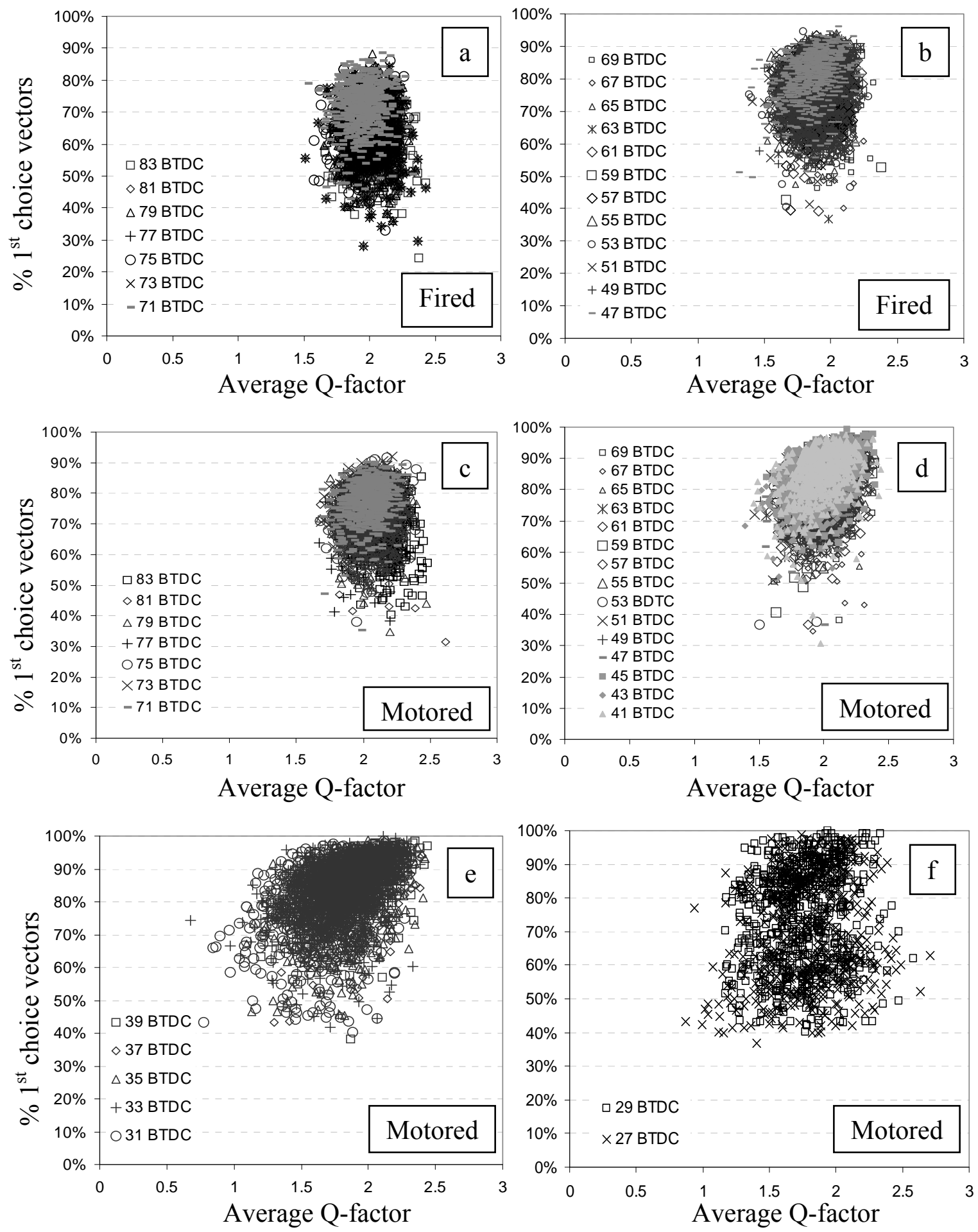

Figure 7-7 Average Q-factor and percentage of first choice vectors for the motored and fired engine experiments 
As shown in Figure 7-7 f, there is considerable scatter in the percentage of vectors retained after post-processing at $29^{\circ} \mathrm{BTDC}$ and $27^{\circ} \mathrm{BTDC}$. The minimum Q-factor is approximately 1.6 , also lower than for the $69^{\circ} \mathrm{BTDC}$ to $41{ }^{\circ} \mathrm{BTDC}$ range. This trend is representative of both motored and fired experiments. At these crank angles, the area between the spark plug and the piston surface decreases considerably and with it, the number of vectors that can be calculated. If the number of spurious vectors is similar to what is found at larger FOVs, the impact at these crank angles would be more significant. Near TDC, increases in the light scattered off the engine head can decrease the signal-tonoise ratio, which might contribute to the lower Q-factors shown in Figures 7-7 e and f. At $29^{\circ}$ BTDC and $27^{\circ}$ BTDC, only data with Q-factors above 1.5 and validation rates at or above $80 \%$ were used in subsequent calculations.

\subsection{Results and Discussion}

\subsubsection{Velocity Fields}

Velocity fields representative of the in-cylinder flow during the compression stroke of two (motored and fired) SIDI engine cycles will be shown in this section. In all cases the velocity fields have been superimposed onto their corresponding background images to show important features in the field of view such as the spark plug. A black horizontal line has been drawn at the bottom of each image to indicate the piston location.

These velocity data are available at every other crank angle from $69{ }^{\circ}$ BTDC to 27 ${ }^{\circ} \mathrm{BTDC}$ for the motored experiment. As stated earlier, the $45^{\circ} \mathrm{BTDC}$ to $33^{\circ} \mathrm{BTDC}$ range was excluded for the fired experiment because during this time, the spray covers most of the field of view. Only a subset of the data is presented. The selected crank angles

illustrate the nature of the flow during the compression stroke of an SIDI engine and highlight differences between flow characteristics under motored and fired conditions. 


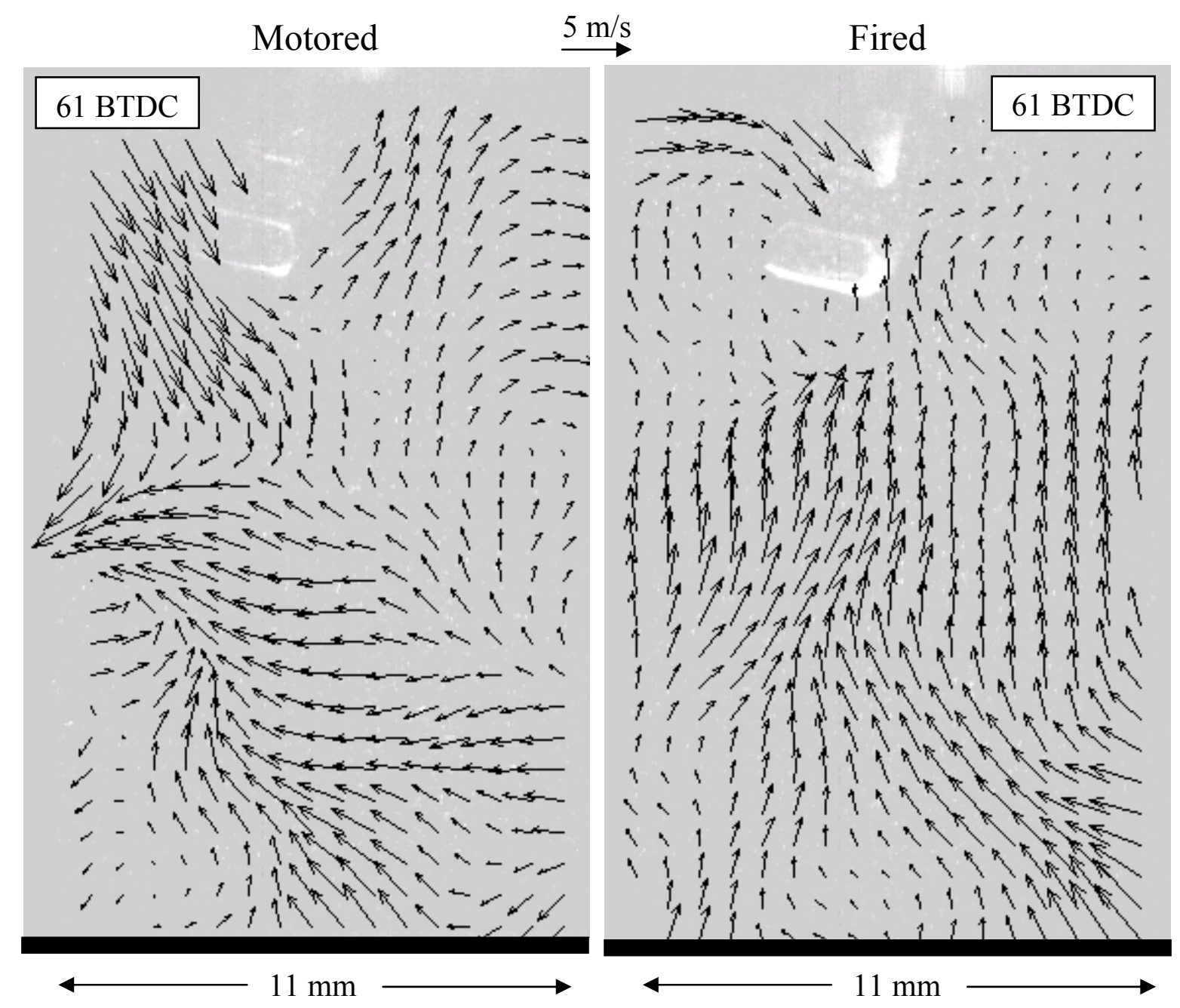

Figure 7-8 Instantaneous flow fields at $61^{\circ} \mathrm{BTDC}$ for motored and fired experiments

Instantaneous velocity fields corresponding to $61{ }^{\circ} \mathrm{BTDC}$ and $57{ }^{\circ} \mathrm{BTDC}$ are shown in Figure 7-8 and Figure 7-9, respectively. In both cases, velocity gradients are present in the $11 \times 15 \mathrm{~mm}^{2}$ area retained for the computations. Vortical structures have been captured at various locations, including areas near the spark plug (Figure 7-9). 


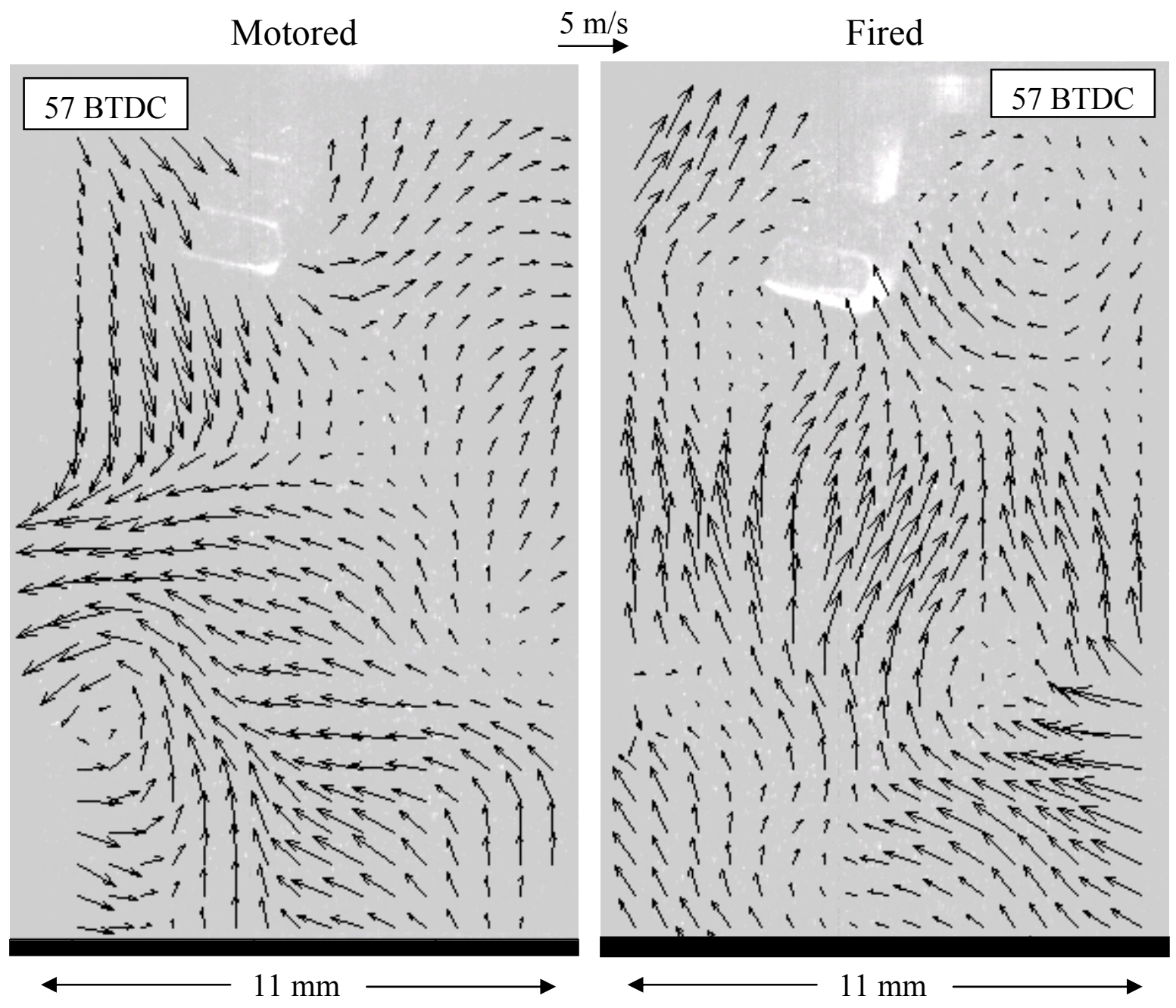

Figure 7-9 Instantaneous flow fields at $57^{\circ} \mathrm{BTDC}$ for motored and fired experiments

As expected, the instantaneous flow fields are not well represented by their ensemble averages (Figure 7-10), since flow structure detail is lost to a large extent. In both (motored and fired cases) the ensemble average indicates that the flow to the left of the spark plug is directed towards the gap. Since the selected measurement plane is located approximately $2 \mathrm{~mm}$ in front of the ground strap (Figure 7-4), it is unclear whether this trend is representative of the average flow motion at the spark plug gap, which has significant influence on the early stages of combustion. As noted earlier (see Chapter 4), the out of plane motion in this tumble-like flow is not expected to be 
significant before the spray arrival, but measurements at multiple planes would be required to fully characterize the flow motion near the spark plug.

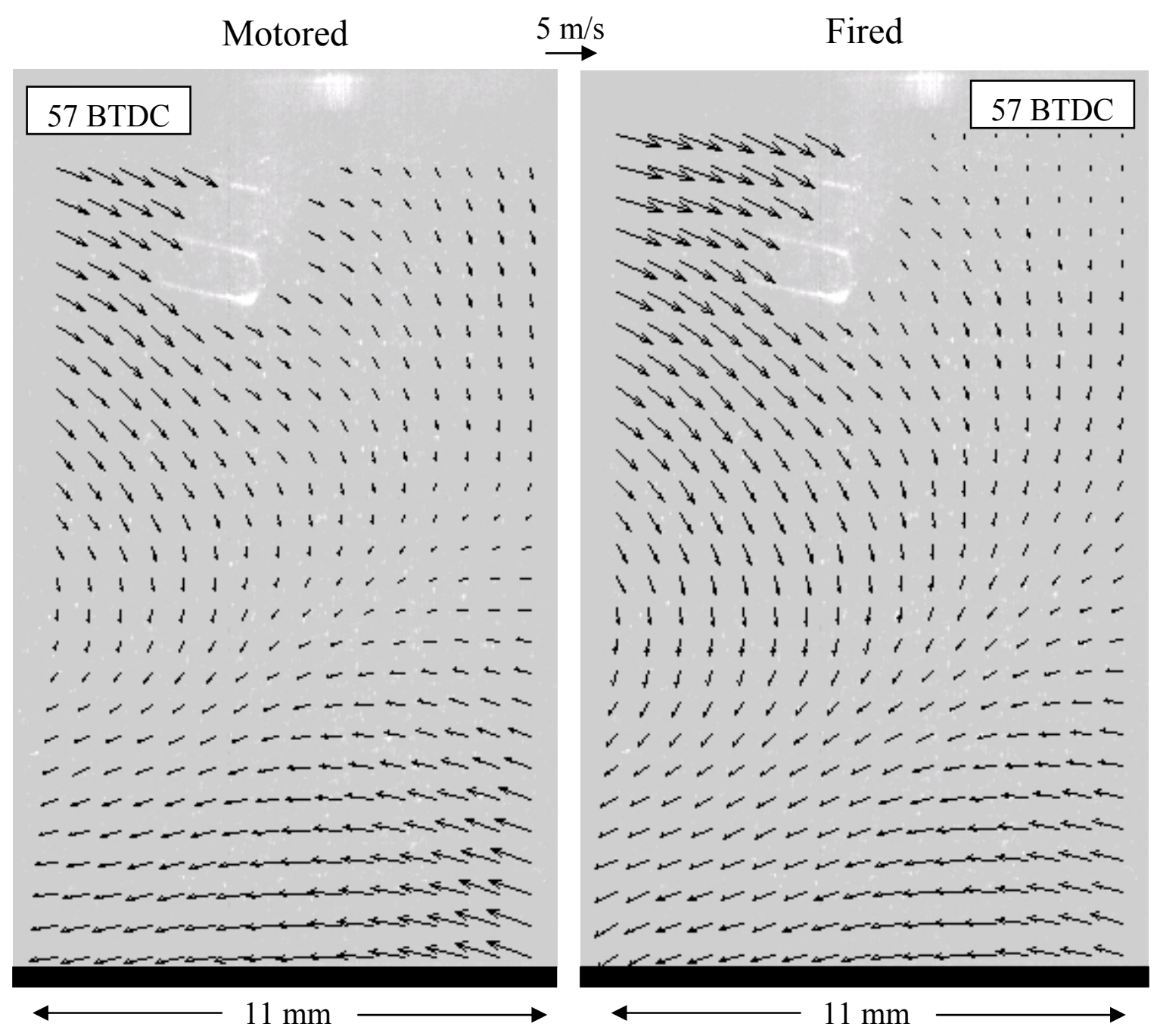

Figure 7-10 Ensemble average flow fields at $57^{\circ} \mathrm{BTDC}$ for motored and fired experiments

The fuel injection event, triggered at $57^{\circ} \mathrm{BTDC}$, is expected to last $17 \mathrm{crank}$ angles $(1.41 \mathrm{~ms})$. The first image of Figure 7-11 shows some of the events taking place less than a millisecond after the fuel-injection triggering event. Unseeded areas to the left of the light sheet, which are excluded during vector computation, have been included in this figure to show the arrival of the spray jets to the FOV. 


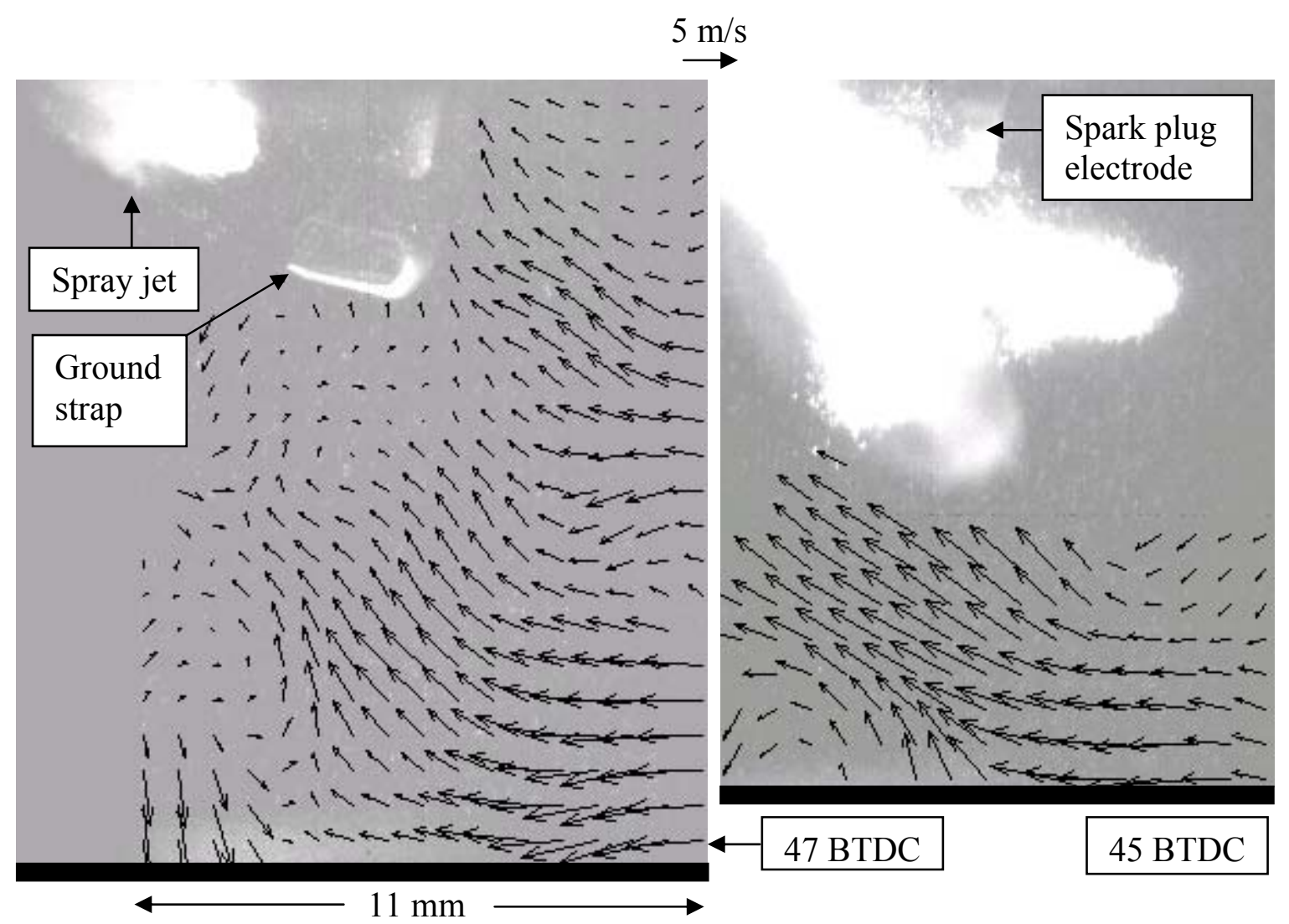

Figure 7-11 Flow fields at $47^{\circ} \mathrm{BTDC}$ and $45^{\circ} \mathrm{BTDC}$, during the arrival of the spray jets to the field of view.

As shown in the first image of Figure 7-11 one of the spray jets is targeted at the spark plug gap and contacts the ground strap between $47^{\circ} \mathrm{BTDC}$ and $45^{\circ} \mathrm{BTDC}$. At 45 ${ }^{\circ} \mathrm{BTDC}$, due to spray penetration, the velocities of the surrounding air can be only obtained on the bottom half of the field of view. From $41{ }^{\circ}$ BTDC to approximately 33 ${ }^{\circ} \mathrm{BTDC}$, the spray jets disperse. During this time, a considerable amount of liquid fuel is still present near the spark plug. The irregular spray shape and dispersion of fuel droplets across the FOV prevents effectively cross-correlating the double-frame images over this crank angle range. 


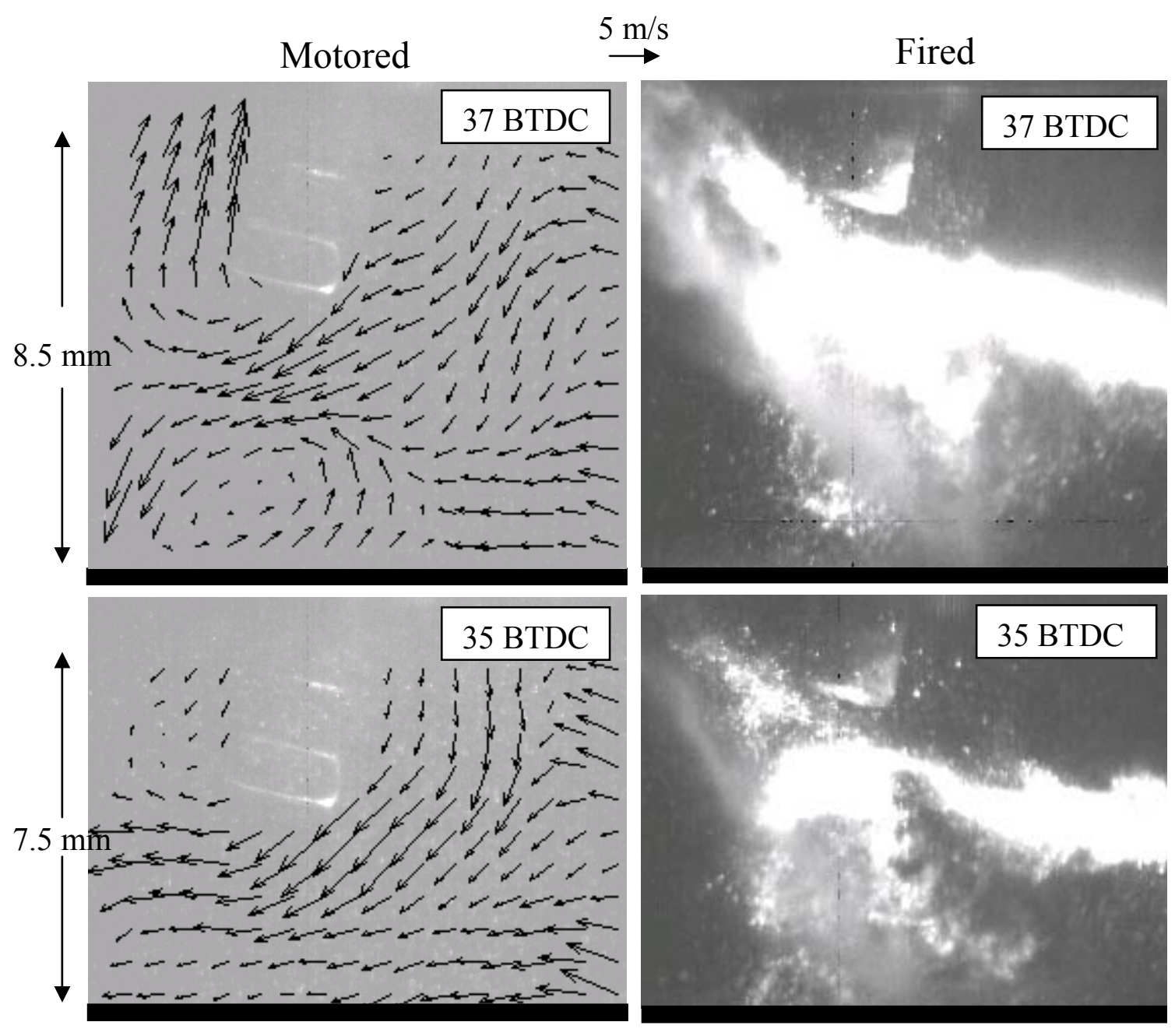

Figure 7-12 Conditions at $37^{\circ} \mathrm{BTDC}$ and $35^{\circ} \mathrm{BTDC}$ for motored (left column) and fired (right column) experiments.

Instantaneous flow fields for the motored engine experiment during this time are also shown in Figure 7-12. Flow structures on the order of $2 \mathrm{~mm}$ persist at $37{ }^{\circ}$ BTDC for this engine cycle.

The flow-field sequences for both the motored and fired experiments are continued on Figure 7-13. In the motored case (left column), the clockwise flow motion persists up to $27^{\circ} \mathrm{BTDC}$, whereas for the fired experiment, the flow velocity near the spark plug region is strongly influenced by the spray (compare Figures 7-14 c and g). The ignition event is triggered at $34{ }^{\circ}$ BTDC. While the luminosity from the spark 
discharge is captured at $33{ }^{\circ} \mathrm{BTDC}$, it can be more clearly seen between $31^{\circ} \mathrm{BTDC}$ and $27^{\circ}$ BTDC (Figure 7-13 f, g and h).

In approximately $84 \%$ of the cycles the plasma channel deformed as seen in Figure 7-13 f, with the distance from the curve peak to the spark plug electrode and ground strap reaching up to $3 \mathrm{~mm}$. In $12 \%$ of the cycles the plasma channel also displaced to the right, but deformed into an S-like shape, as shown Figure 7-14 a. Finally, left motion of the plasma channel was observed only in $4 \%$ of the cycles as the piston traversed from $31^{\circ} \mathrm{BTDC}$ to $27{ }^{\circ} \mathrm{BTDC}$. In these cases, however, the plasma channel remained mostly within the spark plug gap.

Two important issues must be emphasized along with these observations (1) the measurement plane is not centered at the spark plug gap and (2) the out-of-plane motion of the plasma channel cannot be captured using the current two-dimensional measurement technique. The first point is especially relevant since at this stage in the compression stroke, velocity gradients in the direction perpendicular to the measurements plane are expected to increase under the influence of the fuel spray. From the data presented here, it cannot be concluded that there is a direct correlation between the motion of the plasma channel and the velocity field. However, the current measurement demonstrates that the developed technique can be used for investigating the interaction of the plasma channel with the surrounding flow field. 

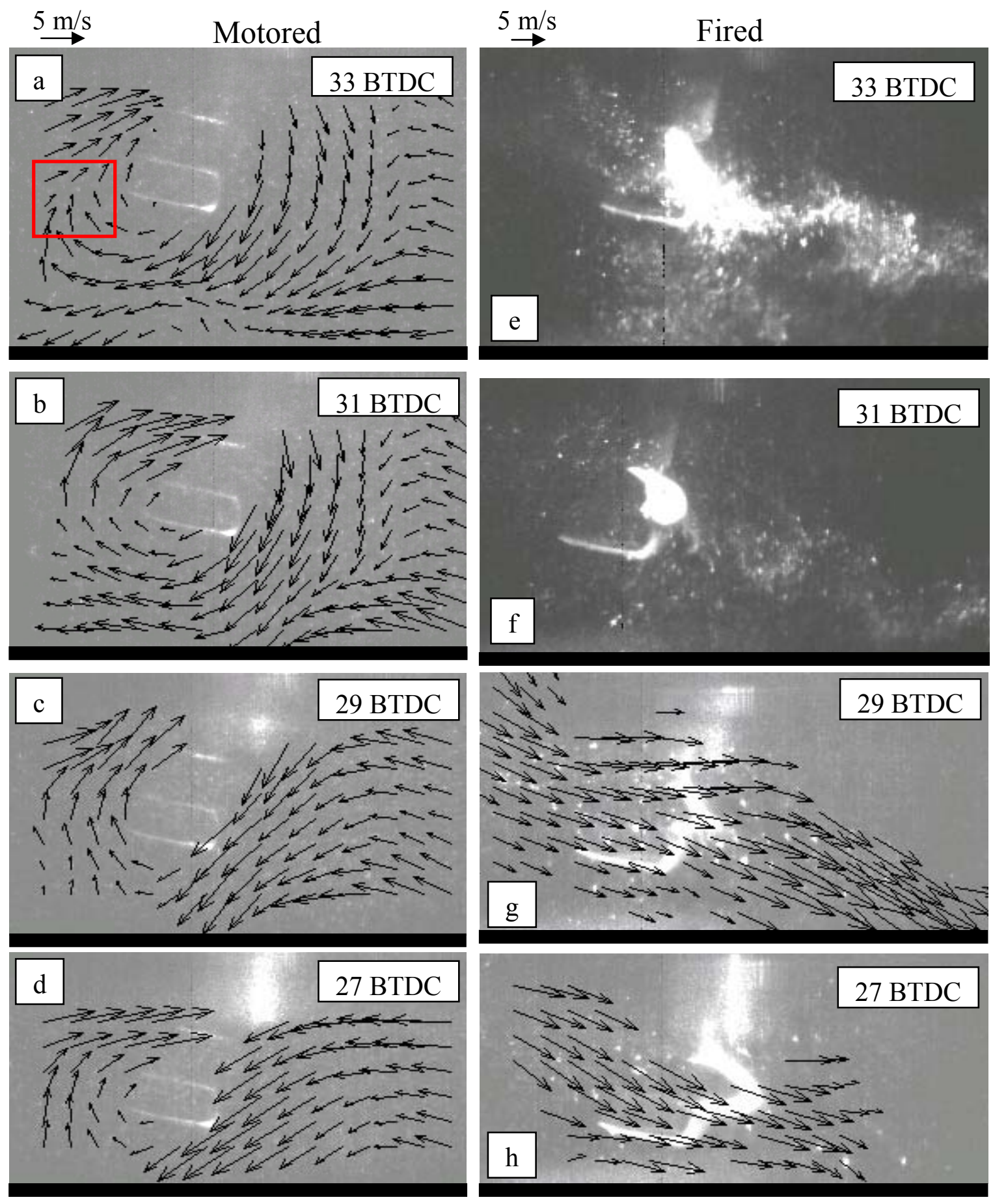

Figure 7-13 Instantaneous flow images between $33^{\circ} \mathrm{BTDC}$ and $27^{\circ} \mathrm{BTDC}$ for a single engine cycle. The spark luminosity is captured at $33^{\circ} \mathrm{BTDC}$. The deformation of the plasma channel can be seen at $29^{\circ} \mathrm{BTDC}$ and $27^{\circ} \mathrm{BTDC}$. 

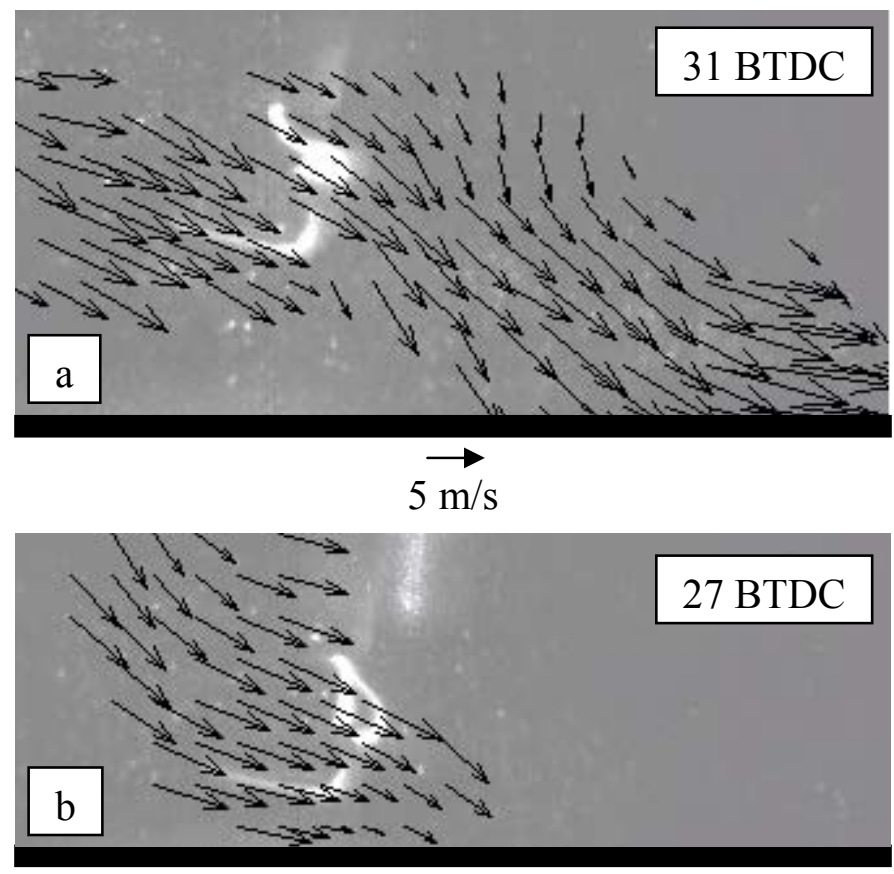

Figure 7-14 Deformation of the plasma channel at $31^{\circ} \mathrm{BTDC}$ and $27^{\circ} \mathrm{BTDC}$

\subsubsection{Kinetic Energy}

To obtain the kinetic energy, the instantaneous velocity fields were decomposed into its ensemble mean and fluctuating components, according to Equation (1-1). At each crank angle, the two-dimensional kinetic energy of the ensemble mean and velocity fluctuations, defined in Equation (6-3) has been calculated and spatially averaged over a $2 \mathrm{~mm}^{2}$ area near the spark plug, which is outlined in Figure 7-13 a. Results for the motored and fired experiments are presented in Figure 7-15 and Figure 7-16. 


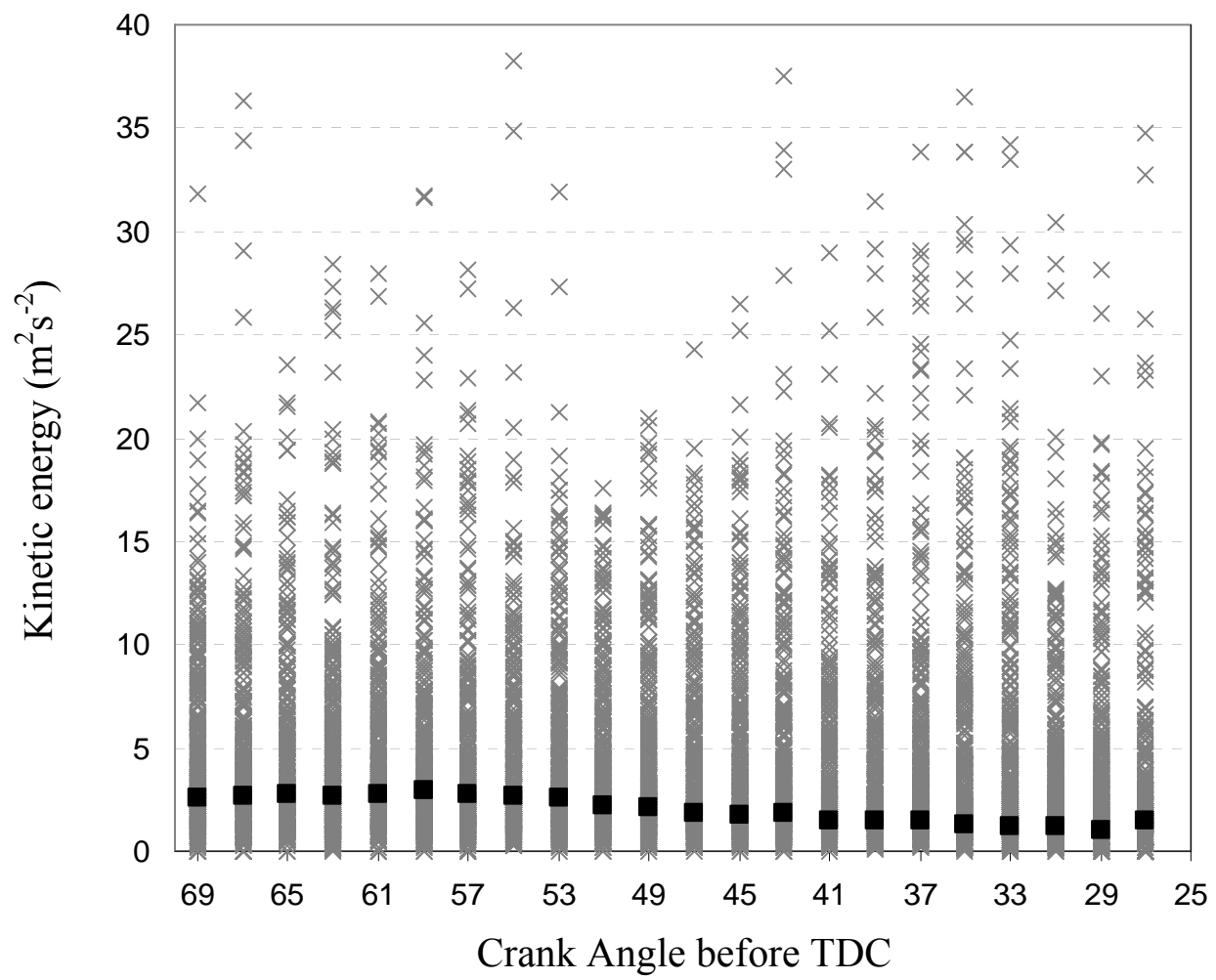

Figure 7-15 Kinetic energy in a $2 \mathrm{~mm}^{2}$ region near the spark plug for the motored-engine experiment. Legend: $\mathrm{x}$-instantaneous values, $\square$ ensemble mean kinetic energy values.

In these figures, the $\mathrm{x}$-symbols represent the instantaneous (spatially-averaged) kinetic energy of the velocity fluctuations, whereas the squares represent the kinetic energy of the ensemble mean at each crank angle. The spatial RMS of the ensemble mean, shown with error bars, remains below $20 \%$ of the ensemble mean kinetic energy at most crank angles in the motored case. For the fired case, the RMS peaks at $27 \%$ of the ensemble mean between $31^{\circ} \mathrm{BTDC}$ and $27^{\circ} \mathrm{BTDC}$. In both the motored and fired engine experiments, instantaneous kinetic energy values greater than $20 \mathrm{~m}^{2} \mathrm{~s}^{-2}$ were accounted for by $3 \%$ of the cycles.

In the motored case, from $69{ }^{\circ} \mathrm{BTDC}$ to $43{ }^{\circ} \mathrm{BTDC}$, the kinetic energy of the velocity fluctuations remained above the kinetic energy of the ensemble mean in $60 \%$ of the cycles. From $41{ }^{\circ} \mathrm{BTDC}$ to $27^{\circ} \mathrm{BTDC}$, the number of cycles for which the kinetic energy of the fluctuations surpassed that of the ensemble mean reached $80 \%$. This is 
consistent with the decrease in ensemble mean kinetic energy shown in Figure 7-15, which has been reproduced in Figure 7-17 for clarity. The decrease in ensemble mean kinetic energy suggests that the energy contained in the velocity fluctuations, whether it corresponds to turbulence only, or to turbulence plus cycle to cycle variability, becomes more relevant as the piston approaches TDC in the motored case. This trend is consistent with previous findings [17].

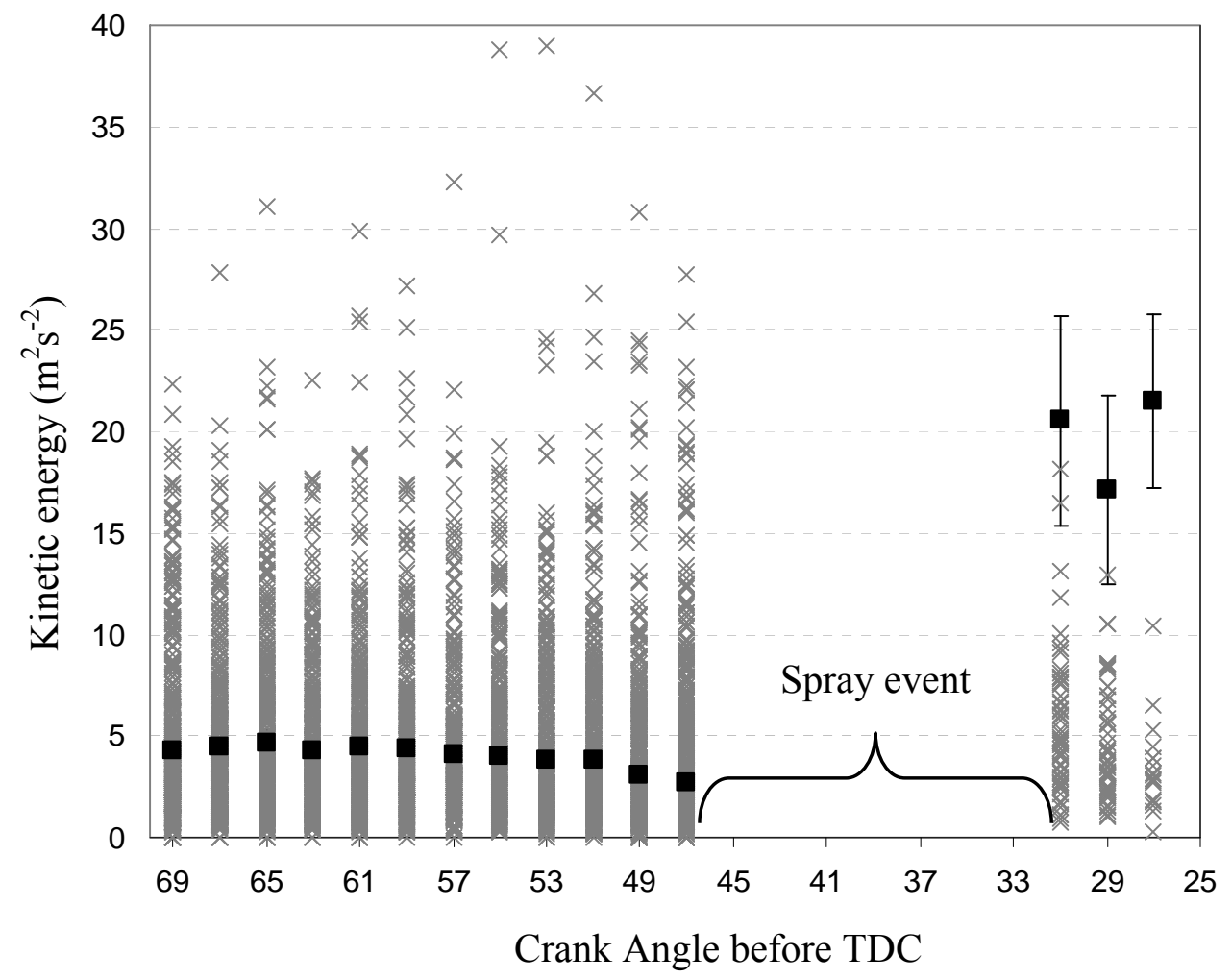

Figure 7-16 Kinetic energy in a $2 \mathrm{~mm}^{2}$ region near the spark plug for the fired-engine experiment. Legend: $\mathrm{x}$-instantaneous values, $\mathbf{a}$ ensemble mean kinetic energy values.

For the fired experiment, the kinetic energy of the ensemble mean decreases from $69^{\circ} \mathrm{BTDC}$ to $47^{\circ} \mathrm{BTDC}$ (refer to Figure $7-16$ ) as it was the case for the motored engine run. As previously shown, the presence of the spray between $47^{\circ} \mathrm{BTDC}$ and $43{ }^{\circ} \mathrm{BTDC}$ precludes the calculation of velocity fields over this crank angle range, as indicated in the figure. 


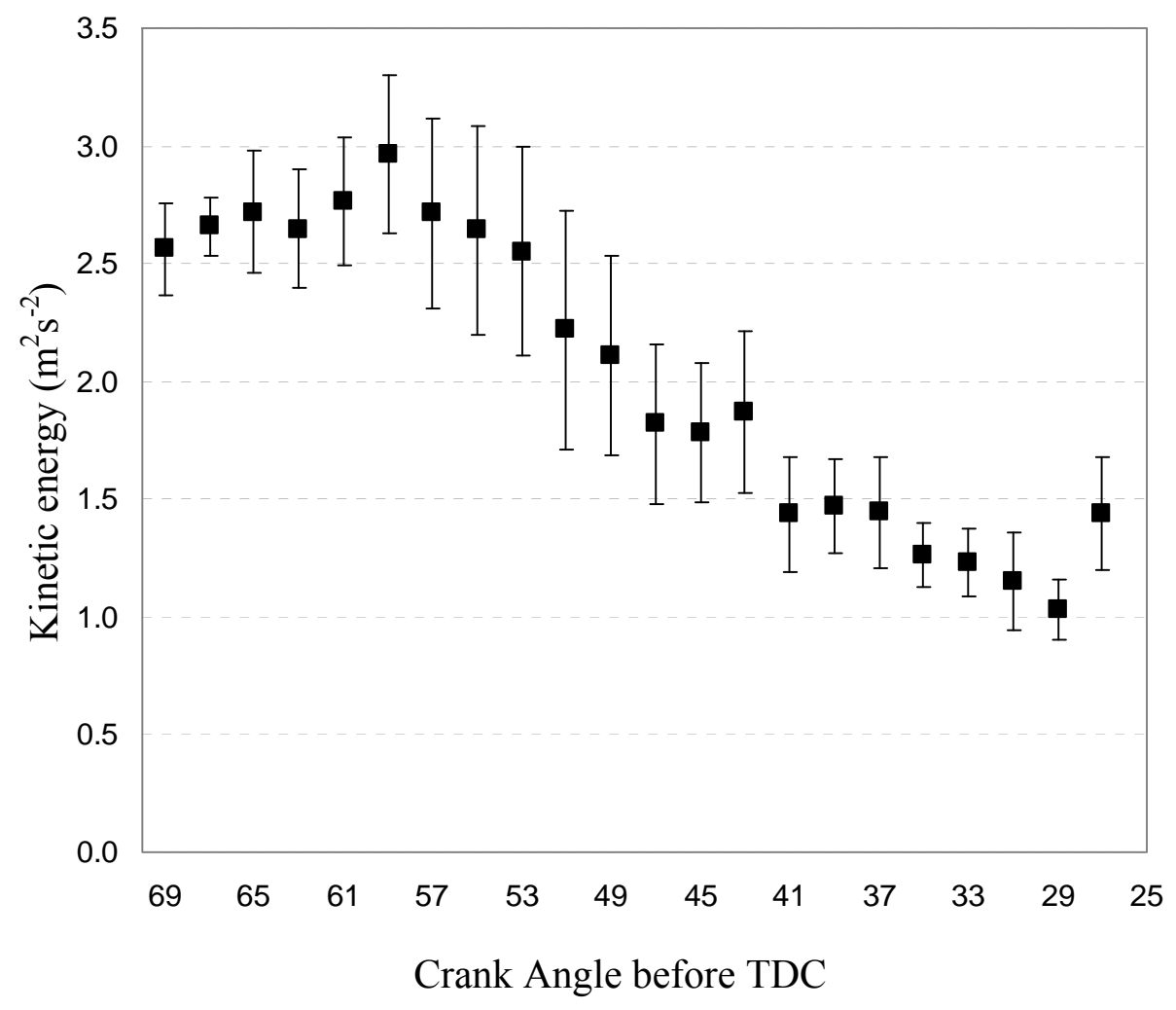

Figure 7-17 Kinetic energy of the ensemble mean averaged over 300 cycles in a $2 \mathrm{~mm}^{2}$ region near the spark plug for the motored-engine experiment

The flow late in the last third of the compression stroke is mainly dominated by the spray event. This was illustrated by the velocity-field sequence of Figure 7-13 and it is also reflected as an increase in the ensemble mean kinetic energy between $31^{\circ} \mathrm{BTDC}$ and $27^{\circ}$ BTDC (Figure 7-16). The spray induces a strongly-directed flow in the vicinity of the spark plug. Results presented in Chapter 4 indicate that this motion persists until the early stages of flame development. The current UV PIV technique could be applied to further investigate the effect of the surrounding flow on near-ignition events in SIDI engines. 


\section{CHAPTER 8}

\section{CONCLUSIONS AND FUTURE WORK}

\subsection{Conclusions}

The development of high-speed particle image velocimetry (PIV) using UV excitation and the first application of the technique for velocity measurements in an SIDI engine operated under motored and fired conditions have been presented. Efforts to develop this technique have stemmed from the need for instantaneous, high-speed velocity measurements to investigate the cycle-specific characteristics of in-cylinder flows and to assess the flow impact on near-ignition events.

The goals, defined in Section 1.3, were (1) to obtain velocity data at multiple crank angles during individual cycles and over multiple consecutive cycles, (2) apply the technique to a production-type stratified SIDI engine and (3) allow flexibility to incorporate high-speed imaging of scalar fields under the same engine operating conditions. The final technique, presented in Chapter 7 meets these requirements. Velocity data have been acquired at the rate of one velocity field at every other crank angle. The technique has been applied to a production-type SIDI engine, operated at 2000 RPM in stratified mode under both motored and fired conditions. Finally, $355 \mathrm{~nm}$ light has been incorporated to facilitate simultaneous high-speed imaging of velocity and fuel equivalence ratio distribution fields using biacetyl PLIF. This technique is novel in that it achieves unprecedented temporal resolution and uses $355 \mathrm{~nm}$ laser light, instead of the $532 \mathrm{~nm}$ excitation wavelength traditionally employed in PIV work. The advantages of using $355 \mathrm{~nm}$ include suppressing the detection of combustion product luminosity and 
capturing the luminosity from the spark discharge. It also enables the integration of biacetyl PLIF for simultaneous quantitative measurements of velocity and scalar fields.

Such simultaneous PIV-PLIF measurements at a sustained $12 \mathrm{kHz}$ acquisition rate have been demonstrated in a cold-air jet. Results showed that there is negligible interaction between biacetyl (PLIF tracer) and the silicon oil droplets used as seeding for PIV. The signal-to-noise ratio and data validation rates of PIV are comparable to traditional setups employing $532 \mathrm{~nm}$ excitation wavelength. Cross-talk between PIV and PLIF signals was approximately 200 counts ( $15 \%$ of the total PLIF signal). A strategy to completely eliminate it was devised and discussed.

The quality of the velocity fields obtained using PIV depends, amongst other factors, on achieving optimum seeding density. For the present UV PIV application in an SIDI engine, this parameter limited the crank angle range over which velocity fields could be acquired with adequate data validation rates. Instantaneous flow fields between $69^{\circ} \mathrm{BTDC}$ and $27^{\circ} \mathrm{BTDC}$ and ensemble averaged flow fields at $57^{\circ} \mathrm{BTDC}$ have been presented for motored and fired-engine cycles. In the instantaneous flow fields, vortical structures persist late in the compression stroke (up to $27^{\circ} \mathrm{BTDC}$ ) across the FOV, including areas immediately surrounding the spark plug. In contrast, flow structures are largely absent in the ensemble-mean flow fields, which also show that the flow to the left of the spark plug follows a clockwise motion predominantly directed towards the gap in both motored-only and fired cases.

The kinetic energy of the ensemble mean and of the velocity fluctuations have been calculated between $69^{\circ} \mathrm{BTDC}$ and $27^{\circ} \mathrm{BTDC}$ for the motored case. For the fired case, velocity (and thus kinetic energy) fields could not be computed between $45{ }^{\circ} \mathrm{BTDC}$ and $31{ }^{\circ} \mathrm{BTDC}$ due to the presence of the high velocity fuel spray in the FOV. The kinetic energy of the ensemble mean was approximately a factor of two higher in the fired case than in the motored-only case from $69^{\circ} \mathrm{BTDC}$ to $47^{\circ} \mathrm{BTDC}$. Over this crank angle range, the kinetic energy of the ensemble mean decreased as the piston approached 
TDC in both experiments. For the fired case, the kinetic energy of the ensemble mean increased by approximately a factor of four after the spray event.

The effect of PIV measurement noise on the kinetic energy and dissipation rate spectra of IC engine flows has been discussed for high and low swirl flows at $60{ }^{\circ} \mathrm{BTDC}$ and 2000 RPM engine speed. Comparisons between low-resolution $(2 \mathrm{~mm})$ and highresolution $(0.35 \mathrm{~mm})$ measurements show an abrupt increase in both energy and dissipation rate spectra at the spatial resolution limit of the measurements. This increase can be attributed to noise introduced by the PIV interrogation algorithm. Three pieces of evidence support this conclusion: (1) the peaks are not coincident at the same scales for high- and low- resolution measurements, (2) they occur at the spatial resolution limit of each setting, (3) they can be found in spectra calculated from fixed-displacement experiments performed with electro-optical image shifting. It has also been shown that a $3 \times 3$ spatial averaging filter applied to the individual vector fields removes the peaks in the spectra, while attenuating kinetic energy and dissipation rate values at the scales of the PIV resolution.

\subsection{Future Work}

This work shows that high-speed UV-PIV can be applied using both a single-laser single-camera arrangement and a two-laser, single camera setup. The former has been used to quantify the velocity and strain rate fields near the spark plug and to visualize the extension of the plasma channel under the action of the surrounding flow. The singlelaser, single-camera technique has also been combined with high-speed PLIF of biacetyl to simultaneously image the flow and equivalence ratio distribution in a cold air jet. Since the two-laser, single camera UV PIV technique has now been demonstrated in the SIDI engine, future work should focus on integrating high-speed UV-PIV and biacetyl PLIF for near-ignition investigations in SIDI engines at 2000 RPM, including the 
interaction of the plasma channel with the surrounding flow field. The combination of high-speed UV PIV and biacetyl PLIF can become a powerful tool for conducting quantitative diagnostic studies in internal combustion engines and similar devices.

An increase in laser power at $355 \mathrm{~nm}$ would have the most significant effect on improving the two-laser, single-camera UV PIV technique. In addition to increasing the signal-to-noise ratio, it would allow for increasing the f-number of the imaging system. This would have two significant effects: (1) it would allow for increasing the depth of field, which would decrease the impact of engine vibrations on light sheet alignment and overlap and (2) it would contribute to reducing the aberration of particle images, which would improve detectability and data validation rates. Until new advances in laser technology become available, these issues could be addressed by decreasing the light sheet width to increase the incident power per unit area and/or by reducing the number optics in the beam path.

Concurrently with the development and application of high-speed UV PIV, considerable effort has been devoted to obtaining kinetic energy and dissipation rate spectra of IC engine flows. This kind of research is important, yet scarce in the IC engine literature. The availability of cycle-resolved velocity data via this high-speed UV particle image velocimetry facilitates not only misfire-related investigations in SIDI engines but also additional research on the evolution of kinetic energy and dissipation rate spectra of IC engine flows. These data can help enhance the current understanding of engine turbulence and contribute to the validation of CFD models used in the design and development of IC engines. 


\section{BIBLIOGRAPHY}

1. Zhao, F., Harrington, D. L., Lai, M-C. (2002). “Automotive Gasoline Direct-Injection Engines." Society of Automotive Engineers, Inc. Warrendale, PA.

2. Zhao, F., Lai, M.-C., et al. (1999). "Automotive Spark-Ignited Direct-Injection Gasoline Engines.” Progress in Energy and Combustion Science 25 (5): 437-562.

3. Kume, T., Iwamoto, Y. et al. (1996). "Combustion Control Technologies for Direct Injection SI Engine.” SAE Paper 960600.

4. Heywood, J. B. "Internal Combustion Engine Fundamentals.” New York, Mc-Graw Hill, Inc.

5. Daneshyar, H., Hill, P. G. (1987). "The Structure of Small-Scale Turbulence and Its Effect on Combustion in Spark-Ignition Engines." Progress in Energy and Combustion Science 13 (1): 47-73.

6. Heywood, J. B. (1987). "Fluid Motion within the Cylinder of Internal Combustion Engines." Transactions of the ASME 109: 1-35.

7. Liou, T. M., Hall., M., et al. (1984). "Laser Doppler Velocimetry Measurements in Valved and Ported Engines." SAE Paper 840375.

8. Winsor, R. E., Patterson, D. J. (1973). "Mixture Turbulence-A Key to Cyclic Combustion Variation.” SAE Paper 730086.

9. Johnston, S. C., Robinson, C. W., et al. (1979). "Application of Laser Diagnostics to an Injected Engine." SAE paper 790092.

10. Mueller, C. H., Driscoll, J. J., et al. (1998). "Vorticity Generation and Attenuation as Vortices Convect through a Premixed Flame." Combustion and Flame. 112: 342-358.

11. Peters, N. Turbulent Combustion. Cambridge University Press, Cambridge, 2000.

12. Pischinger, S., Heywood, J. B. (1990). "How Heat Losses to the Spark Plug Electrodes Affect Flame Kernel Development in an SI Engine." SAE Paper 900021.

13. Patterson, D. J. (1967). “Cylinder Pressure Variations, a Fundamental Combustion Problem.” SAE Paper 660129. 
14. Reuss, D.L. (2000). "Cyclic Variability of Large-Scale Turbulent Structures in Directed and Undirected IC Engine Flows.” SAE Paper 2000-01-0246.

15. Reuss, D.L., Kuo, T-W., et al. (1995). "Particle Image Velocimetry Measurements in a High-Swirl Engine Used for Evaluation of Computational Fluid Dynamics Calculations.” SAE Paper 952381.

16. Lumley, J. L. (2001). "Early Work on Fluid Mechanics in the IC Engine.” Annual Review of Fluid Mechanics 33: 319-338.

17. Arcoumanis, C., Hu, Z., et al. (1991). "Tumbling Motion: a Mechanism for Turbulence Enhancement in Spark-Ignition Engines.” SAE paper 900060.

18. Tennekes, H., and J.L. Lumley. A First Course in Turbulence. The MIT Press, Cambridge, MA, 1972.

19. Reuss, D. L., Adrian, R. J. (1989). “Instantaneous Planar Measurements of Velocity and Large-Scale Vorticity and Strain Rate in an Engine Using Particle-Image Velocimetry." SAE Paper 890616.

20. Reuss, D. L., Rosalik, M. (2000). "PIV Measurements During Combustion in a Reciprocating Internal Combustion Engine." Laser Techniques Applied to Fluid Mechanics: 441-456.

21. Towers D. P., Towers, C. E. (2004). "Cyclic Variability Measurements of In-Cylinder Engine Flows Using High-Speed Particle Image Velocimetry.” Measurement Science and Technology 15: 1917-1925.

22. Jarvis S., Justham, T., et al. (2006). "Time Resolved Digital PIV Measurements of Flow Field Cyclic Variation in an Optical IC Engine.” SAE Paper 2006-01-1044.

23. Stahl, G., Warnatz, J. (1991). "Numerical Investigation of Time-Dependent Properties and Extinction of Strained Methane- and Propane-Air Flamelets." Combustion and Flame 85: 285-299

24. Reeves, M., Towers, D. P., et al. (1999). “A High-Speed All-Digital Technique for Cycle-Resolved 2-D Flow Measurements and Flow Visualization within SI Engine Cylinders." Optics and Lasers in Engineering. 31: 247-261.

25. Ghandhi, J. B., Herold, R. E., et al. (2005). "Time-Resolved Particle Image Velocimetry Measurements in an Internal Combustion Engine." SAE Paper 2005-01$\underline{3868}$.

26. Raffel, M., Willert, C., and C. Kompenhans. "Particle Image Velocimetry", SpringerVerlag, 1998. 
27. Westerweel, J. (1997). "Fundamentals of Digital Particle Image Velocimetry." Measurement Science and Technology 8: 1379-1392.

28. Westerweel, J., Dabiri, D., Gharib, M. (1997). "The Effect of a Discrete Window Offset on the Accuracy of Cross-Correlation Analysis of Digital PIV Recordings." Experiments in Fluids 23: 2-28.

29. Keane, R. D., Adrian, R. J. (1990). “Optimization of Particle Image Velocimeters. Part I: Double Pulsed Systems.” Measurement Science and Technology 1: 1202-1215, 1990.

30. Megerle, M., Sick V., Reuss D. L. (2002). "Measurement of Digital Particle Image Velocimetry Precision Using Electro-Optically Created Particle-Image Displacements." Measurement Science and Technology 13: 997-1005.

31. Reuss D. L., Megerle, M., Sick V. (2002). "Particle-Image Velocimetry Measurement Errors When Imaging through a Transparent Engine Cylinder.” Measurement Science and Technology 13: 1029-1035.

32. Funk, C.O. PhD Thesis. University of Michigan, 2005.

33. Melling, A. (1997). "Tracer Particles and Seeding for Particle Image Velocimetry." Measurement Science and Technology 8: 1406-1416.

34. Fissenewert, U., Sick, V., Pucher, H. (2005). "Characterization of Combustion and NO Formation in a Spray-Guided, Gasoline, Direct-Injection Engine Using Chemiluminescence Imaging, NO-PLIF, and Fast NO Exhaust Gas Analysis." SAE Paper 2005-01-2089.

35. Adrian, R. J. (1991) "Particle-Imaging Techniques for Experimental Fluid Mechanics." Annual Review of Fluid Mechanics 23: 261-304.

36. Bowditch, F.W., “A New Tool for Combustion Research. A Quartz Piston Engine.”

37. Smith, J. D., Sick, V. (2005). "Crank-Angle Resolved Imaging of Biacetyl LaserInduced Fluorescence in an Optical Internal Combustion Engine.” Applied Physics B 81 579-584.

38. Smith, J. D., Sick, V. (2007). “Quantitative, Dynamic Fuel Distribution Measurements in Combustion-Related Devices Using Laser-Induced Fluorescence Imaging of Biacetyl in Iso-Octane.” Proc. Combust. Inst. 31 (1): 747-755.

39. Driscoll, K. D., Sick, V., Gray, C. (2003). "Simultaneous Air/Fuel-Phase PIV Measurements in a Dense Fuel Spray.” Experiments in Fluids 35: 112-113 
40. Adrian, R. J. (1997). "Dynamic Ranges of Velocity and Spatial Resolution of Particle Image Velocimetry." Measurement Science and Technology 8: 1393-1398.

41. Gajdeczko, B. F., Bracco, F. V. (1999). “Application of Two-Color Particle Image Velocimetry to a Firing Production Direct-Injection, Stratified-Charge Engine.” SAE Paper 1999-01-1111.

42. Fajardo, C. M., Smith, J. D., Sick, V. (2006). "PIV, High-Speed PLIF and Chemiluminescence Imaging for Near-Spark-Plug Investigations in IC Engines." Journal of Physics: Conference Series 45: 19-26.

43. Reuss, D.L., Bardsley, M., et al. (1990). "Velocity, Vorticity, and Strain-Rate Ahead of a Flame Measured in an Engine Using Particle Image Velocimetry." SAE Paper 900053.

44. Funk, C., Sick, V., Reuss, D. L., and Dahm, W. J. A. "Turbulence Properties of High and Low Swirl In-Cylinder Flows.” SAE Paper 2002-01-2841.

45. Willert, C.E., Gharib, M. "Digital Particle Image Velocimetry." Experiments in Fluids 10: 181-193, 1991.

46. Nogueira, J., Lecuona A., Rodriguez, P. A. (2001). "Identification of a New Source of Peak Locking, Analysis and its Removal in Conventional and Super-Resolution PIV Techniques." Experiments in Fluids 30: 309-316. 